\title{
Triacetonide of Glucoheptonic Acid in the Scalable Syntheses of D-Gulose, 6-Deoxy-D-Gulose, L-Glucose, 6-Deoxy-L-Glucose and Related Sugars
}

Zilei Liu, ${ }^{\mathrm{a}, \mathrm{c}}$ Akihide Yoshihara, ${ }^{\mathrm{b} *}$ Sarah F. Jenkinson, ${ }^{\mathrm{a}}$ Mark R. Wormald, ${ }^{\mathrm{c}}$ Ramón J. Estévez, George W. J. Fleet ${ }^{\mathrm{a} *}$, Ken Izumori ${ }^{\mathrm{b}}$

${ }^{a}$ Chemistry Research Laboratory, Department of Chemistry, University of Oxford, Oxford, OX1 3TA, UK

${ }^{\mathrm{b}}$ International Institute of Rare Sugar Research and Education, Kagawa University, Miki, Kagawa 761-0795, Japan

${ }^{c}$ Oxford Glycobiology Institute, Department of Biochemistry, University of Oxford, Oxford, OX1 3QU, UK

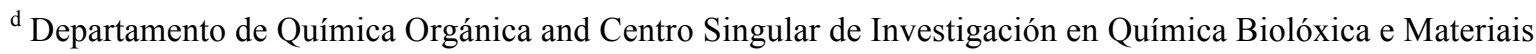

Moleculares, Universidade de Santiago de Compostela, 15782 Santiago de Compostela, Spain

*george.fleet@,chem.ox.ac.uk; yoshihara@,ag.kagawa-u.ac.jp

\section{Supporting Information}

\section{Contents}

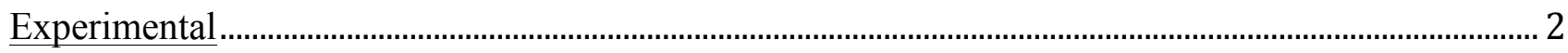

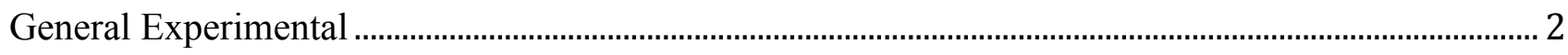

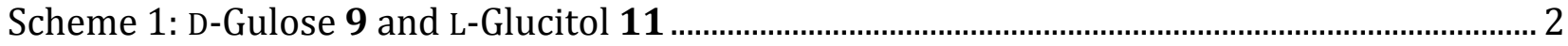

Scheme 2: 6-Deoxy-D-gulonolactone 15, 6-Deoxy-D-gulose 18 and 6-Deoxy-D-gulitol

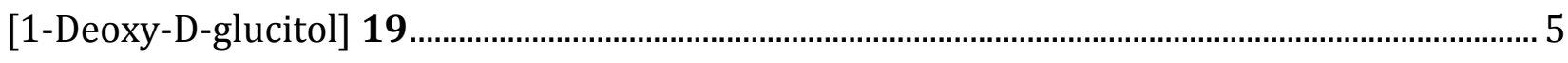

Scheme 3: meso-D-glycero-D-gulo-Heptitol 22, 6-Deoxy-L-glucose [L-quinovose] 31 and 6-Deoxy-L-glucitol 33 8

NMR Spectra 16 


\section{Experimental}

\section{General Experimental}

All commercial reagents were used as supplied. Solvents were used as supplied (Analytical or HPLC grade), without prior purification. Thin layer chromatography (TLC) was performed on aluminium sheets coated with $60 \mathrm{~F}_{254}$ silica. Plates were visualised using a $0.2 \% \mathrm{w} / \mathrm{v}$ cerium (IV) sulfate and $5 \%$ ammonium molybdate solution in $2 \mathrm{M}$ sulfuric acid. Melting points were recorded on a Kofler hot block and are uncorrected. Optical rotations of the protected sugars were recorded on a Perkin-Elmer 241 polarimeter with a path length of $1 \mathrm{dm}$; optical rotation are quoted in $10^{3} \mathrm{deg} \cdot \mathrm{cm}^{2} \cdot \mathrm{g}^{-1}$ at concentrations (c) in $\mathrm{g} \cdot 100 \mathrm{~mL}^{-1}$. Infrared spectra were recorded on a Perkin-Elmer 1750 IR Fourier Transform spectrophotometer using thin films on a diamond ATR surface (thin film). Only the characteristic peaks are quoted. Nuclear magnetic resonance (NMR) spectra were recorded on a Bruker AMX $500\left({ }^{1} \mathrm{H}: 500 \mathrm{MHz}\right.$ and $\left.{ }^{13} \mathrm{C}: 125.7 \mathrm{MHz}\right)$ or Bruker AVIII $400 \mathrm{HD}$ nanobay and Bruker DQX $400\left({ }^{1} \mathrm{H}: 400 \mathrm{MHz},{ }^{13} \mathrm{C}: 100.6 \mathrm{MHz}\right)$ spectrometers in the deuterated solvent stated. ${ }^{1} \mathrm{H}$ and ${ }^{13} \mathrm{C}$ NMR spectra were assigned by utilizing 2D COSY, HSQC and HMBC spectra. All chemical shifts $(\delta)$ are quoted in ppm and coupling constants $(J)$ in Hz. Residual signals from the solvents were used as an internal reference. For solutions in $\mathrm{D}_{2} \mathrm{O}$ acetonitrile was used as an internal reference. HRMS measurements were made using a microTOF mass analyzer using electrospray ionization (ESI). Nomenclature of alditols can be confusing: both glucitol and gulitol names are giving for the avoidance of confusion. Commercial rare sugar samples are kindly provided by Carbosynth Limited.

\section{Scheme 1: D-Gulose 9 and L-Glucitol 11}

\section{Methyl 2,3:4,5-di-O-isopropylidene-D-gulonate 7}<smiles>CC(=O)C1OC(C)(C)OC1C1OC(C)(C)OC1CO</smiles>

Sodium borohydride $(977.0 \mathrm{mg}, 25.70 \mathrm{mmol}$ ) was added in portions to a solution of aldehyde 5 (13.50 g, $46.80 \mathrm{mmol})$ in methanol $(250 \mathrm{~mL})$ at $0{ }^{\circ} \mathrm{C}$. After stirring at $0{ }^{\circ} \mathrm{C}$ for 1.5 hours, TLC (cyclohexane/ethyl acetate 1:1) indicated the formation of product $\left(\mathrm{R}_{\mathrm{f}} 0.40\right)$; glacial acetic acid $(\sim 2.5$ $\mathrm{mL}$ ) was added dropwise to adjust to $\mathrm{pH} 7$. The solvent was removed in vacuo to give a residue that was suspended in water $(100 \mathrm{~mL})$. After washing with ethyl acetate ( $3 \times 100 \mathrm{~mL})$, organic phase was dried $\left(\mathrm{MgSO}_{4}\right)$, filtered and solvent was removed in vacuo to obtain pure product $\mathbf{7}$ as a white solid without further purification $(13.04 \mathrm{~g}, 96 \%)$.

m.p. $76^{\circ} \mathrm{C}-78^{\circ} \mathrm{C} ;[\alpha]_{\mathrm{D}}^{20}=+8.9(c, 1.20$ in $\mathrm{MeOH}) ; v_{\max }$ (thin film): $3502(\mathrm{br}, \mathrm{OH}), 1764(\mathrm{~s}, \mathrm{C}=0) ;{ }^{1} \mathrm{H}$ NMR 
$\left(\mathrm{CDCl}_{3}, 400 \mathrm{MHz}\right) 1.34\left(3 \mathrm{H}, \mathrm{s}, \mathrm{CH}_{3}\right), 1.37\left(6 \mathrm{H}, \mathrm{s}, 2 \times \mathrm{CH}_{3}\right), 1.60\left(3 \mathrm{H}, \mathrm{s}, \mathrm{CH}_{3}\right), 2.55(1 \mathrm{H}, \mathrm{br}-\mathrm{s}, \mathrm{OH}), 3.66(1 \mathrm{H}$, dd, H6, J6,5 3.6, Jgem 12.0), $3.71\left(3 \mathrm{H}, \mathrm{s}, \mathrm{OCH}_{3}\right), 3.82$ (1H, dd, H6', J6,5 3.6, Jgem 12.0), 4.12 (1H, dd, H4, J4,3 2.0, $J_{4.5} 8.0$ ), $4.21\left(1 \mathrm{H}, \mathrm{dt}, \mathrm{H} 5, J_{5,6}=J_{5,6^{\prime}} 3.6, J_{5,4} 8.0\right), 4.36\left(1 \mathrm{H}, \mathrm{dd}, \mathrm{H} 3, J_{3,4} 2.0, J_{3.2} 7.6\right), 4.66\left(1 \mathrm{H}, \mathrm{d}, \mathrm{H} 2, J_{2,3} 7.6\right.$ ); ${ }^{13} \mathrm{C}$ NMR $\left(\mathrm{CDCl}_{3}, 100 \mathrm{MHz}\right) 25.9\left(\mathrm{CH}_{3}\right), 26.7\left(\mathrm{CH}_{3}\right), 26.8\left(\mathrm{CH}_{3}\right), 27.6\left(\mathrm{CH}_{3}\right), 52.5\left(\mathrm{OCH}_{3}\right), 61.9(\mathrm{C} 6), 74.7$ (C4), 75.6 (C5), 76.4 (C2), 77.8 (C3), $110.0\left(\underline{\mathrm{C}}\left(\mathrm{CH}_{3}\right)_{2}\right), 111.4\left(\underline{\mathrm{C}}\left(\mathrm{CH}_{3}\right)_{2}\right), 170.8$ (C1); m/z (ESI+ve): 313 $\left([\mathrm{M}+\mathrm{Na}]^{+}, 100 \%\right) ; \mathrm{HRMS} m / z$ (ESI+ve): found $313.1252[\mathrm{M}+\mathrm{Na}]^{+}, \mathrm{C}_{13} \mathrm{H}_{22} \mathrm{O}_{7} \mathrm{Na}^{+}$requires 313.1257 .

\section{2,3:4,5-Di-O-isopropylidene-D-gulose 8}<smiles>CC1OC(C)(C)OC1C1OC(C)(C)OC1CO</smiles>

Diisobutylaluminum hydride DIBALH (1.5 M in toluene, $75.00 \mathrm{ml}, 112.50 \mathrm{mmol}$ ) was added dropwise to a solution of the methyl ester $7(13.04 \mathrm{~g}, 45.00 \mathrm{mmol})$ in dichloromethane $(70 \mathrm{~mL})$ at $-78{ }^{\circ} \mathrm{C}$. The reaction mixture was stirred at $-78^{\circ} \mathrm{C}$ for 1.5 hours until TLC (cyclohexane/ethyl acetate 1:1) indicated the formation of one major product $\left(\mathrm{R}_{\mathrm{f}} \mathrm{0.43}\right)$. Also mass spectrometry showed the formation of desired product peak $([\mathrm{M}+\mathrm{MeOH}+\mathrm{Na}]+315)$ and disappearance of starting material peak $([\mathrm{M}+\mathrm{Na}]+313)$. The reaction mixture was diluted with ethyl acetate $(100 \mathrm{~mL}$ ) and potassium sodium tartrate (sat. aq. 50 $\mathrm{mL}$ ) was added. After stirring for $8 \mathrm{~h}$, the reaction mixture was diluted with water $(100 \mathrm{~mL})$ and extracted with ethyl acetate $(3 \times 150 \mathrm{~mL})$. The organic phase was dried $\left(\mathrm{MgSO}_{4}\right)$, filtered and the solvent removed in vacuo to obtain a crude residue that was purified by flash chromatography (cyclohexane/ethyl acetate 5:1 $\rightarrow 4: 1$ ) to yield the hydrated form of aldehyde 8 as a syrup (10.00 g, $86 \%)$.

$[\alpha]_{\mathrm{D}}{ }^{20}=+5.4\left(c, 0.82\right.$ in $\left.\mathrm{CHCl}_{3}\right), v_{\max }\left(\right.$ thin film, $\left.\mathrm{cm}^{-1}\right): 3427$ (broad, $\left.\mathrm{OH}\right) ;{ }^{1} \mathrm{H}$ NMR $\left(\mathrm{CDCl}_{3}, 400 \mathrm{MHz}\right) 1.38$ $\left(3 \mathrm{H}, \mathrm{s}, \mathrm{CH}_{3}\right), 1.41\left(3 \mathrm{H}, \mathrm{s}, \mathrm{CH}_{3}\right), 1.43\left(3 \mathrm{H}, \mathrm{s}, \mathrm{CH}_{3}\right), 1.52\left(3 \mathrm{H}, \mathrm{s}, \mathrm{CH}_{3}\right), 3.55$ (1H, dd, H6, J6,5 10.3, Jgem 11.2), $3.67\left(1 \mathrm{H}, \mathrm{ddd}, \mathrm{H} 5, J_{5,6^{\prime}} 4.0, J_{5,4} 9.3, J_{5,6} 10.0\right), 3.88\left(1 \mathrm{H}, \mathrm{t}, \mathrm{H} 4, J_{4,5}=J_{4,3} 9.2\right), 4.11\left(1 \mathrm{H}, \mathrm{t}, \mathrm{H} 2, J_{2,1}=J_{2,3} 8.0\right.$ ), $4.28\left(1 \mathrm{H}, \mathrm{dd}, \mathrm{H6} 6^{\prime}, J_{6^{\prime}, 5} 4.0, J_{\text {gem }} 11.2\right), 4.39$ (1H, dd, H3, J3,2 8.0, J3.4 8.7), 4.75 (1H, d, H1, J1,2 8.2); ${ }^{13} \mathrm{C}$ NMR $\left(\mathrm{CDCl}_{3}, 100 \mathrm{MHz}\right) 24.1\left(\mathrm{CH}_{3}\right), 27.0\left(\mathrm{CH}_{3}\right), 27.1\left(\mathrm{CH}_{3}\right), 27.2\left(\mathrm{CH}_{3}\right), 61.9(\mathrm{C} 6), 74.8(\mathrm{C} 4), 76.9$ (C5), $79.6(\mathrm{C} 3)$, 81.4 (C2), 98.1 (C1), $110.0\left(\underline{\mathrm{C}}\left(\mathrm{CH}_{3}\right)_{2}\right), 111.1\left(\underline{\mathrm{C}}\left(\mathrm{CH}_{3}\right)_{2}\right) ; \mathrm{m} / z$ (ESI+ve): $315\left([\mathrm{M}+\mathrm{MeOH}+\mathrm{Na}]^{+}, 100 \%\right)$; HRMS $m / z$ (ESI+ve): found $283.1149[\mathrm{M}+\mathrm{Na}]^{+}, \mathrm{C}_{12} \mathrm{H}_{20} \mathrm{O}_{6} \mathrm{Na}^{+}$requires 283.1152 .

\section{D-Gulose 9}<smiles>O=CC(O)C(O)C(O)CO</smiles>

DOWEX@ 50WX8-200 (5.00 g) was added to a solution of aldehyde 8 (10.0 g, $38.5 \mathrm{mmol})$ in water (120 $\mathrm{mL}$ ). After stirring at room temperature for 48 hours, mass spectrometry indicated that the reaction was complete and the resin was filtered off; water was removed in vacuo to yield pure D-gulose $\mathbf{9}$ as a colorless syrup without the need for further purification [gulose crystallised over a period of months] 
(6.90 g, 100\%).

$[\alpha]_{\mathrm{D}} 20=-20.0\left(c, 0.89\right.$ in water, eq) [Commercial sample: $[\alpha]_{\mathrm{D}}^{20}=-23.0(c 1.40$ in water, eq $)$, lit. $^{1}[\alpha]_{\mathrm{D}^{20}}=$ -24.1]; $v_{\max }$ (thin film): 3309 (br, OH); ${ }^{1} \mathrm{H}$ and ${ }^{13} \mathrm{C}$ NMR are identical with that of commercial D-gulose

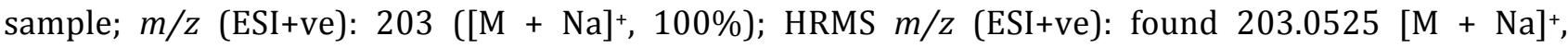
$\mathrm{C}_{6} \mathrm{H}_{12} \mathrm{O}_{6} \mathrm{Na}^{+}$requires 203.0526 .

\section{2,3:4,5-Di-O-isopropylidene-L-glucitol 10}<smiles>CC1(C)OC(CO)C(C2OC(C)(C)OC2CO)O1</smiles>

Lithium aluminum hydride solution (1 $\mathrm{M}$ in THF, $9.4 \mathrm{~mL}, 9.4 \mathrm{mmol}$ ) was added dropwise to a stirred solution of aldehyde $5(1.34 \mathrm{~g}, 4.7 \mathrm{mmol})$ in THF $(40 \mathrm{~mL})$ at $0{ }^{\circ} \mathrm{C}$. The reaction mixture was stirred at room temperature for 2 hours after which TLC analysis (ethyl acetate) showed formation of a major product $\left(\mathrm{R}_{\mathrm{f}} 0.30\right)$. The excess hydride was quenched by dropwise addition of $\mathrm{NH}_{4} \mathrm{Cl}(\sim 2 \mathrm{~mL}$, sat. aq.) at $0{ }^{\circ} \mathrm{C}$; the reaction mixture was dried $\left(\mathrm{MgSO}_{4}\right)$, filtered (washing with ethyl acetate) and concentrated in vacuo to give a residue that was dissolved in ethyl acetate $(50 \mathrm{~mL})$ and washed with water $(2 \times 50 \mathrm{~mL})$. The organic phase was dried $\left(\mathrm{MgSO}_{4}\right)$, filtered and solvent was removed in vacuo to give $\mathbf{1 0}$ which was purified by flash chromatography (ethyl acetate) to yield the protected L-glucitol $\mathbf{1 0}(1.1 \mathrm{~g}, 90 \%)$ as a clear oil.

$[\alpha]_{\mathrm{D}^{20}}=+62.1(c, 1.24 \mathrm{in} \mathrm{MeOH}) ;{ }^{1} \mathrm{H}$ NMR $(400 \mathrm{MHz}, \mathrm{MeOH}) 1.36\left(3 \mathrm{H}, \mathrm{s}, \mathrm{CH}_{3}\right), 1.38\left(3 \mathrm{H}, \mathrm{s}, \mathrm{CH}_{3}\right), 1.40(3 \mathrm{H}$, s, $\left.\mathrm{CH}_{3}\right), 1.48\left(3 \mathrm{H}, \mathrm{s}, \mathrm{CH}_{3}\right), 3.64\left(1 \mathrm{H}, \mathrm{dd}, \mathrm{H} 1, J_{1,2} 4.9, J_{\text {gem }} 11.9\right), 3.70$ (1H, dd, H1', J',2 4.3, Jgem 11.9), 3.78 (1H, dd, H6, J6,5 4.9, Jgem 11.3), 3.83 (1H, dd, H6', J6,5 6.7, Jgem 11.3), 3.88 (1H, dd, H3, J3,4 0.7, J3,2 8.5), 4.07 (1H, ddd, H2, J2,1 4.3, J2,1 4.9, Jgem 8.5), 4.21 (1H, dd, H4, J4,3 0.7, J4.5 6.4), 4.35 (1H, ddd, H5, J5,6 4.9, J5,4 6.4, $\left.J_{5,6}, 6.7\right) ;{ }^{13} \mathrm{C}$ NMR (100MHz, MeOH) $24.2\left(\mathrm{CH}_{3}\right), 25.8\left(\mathrm{CH}_{3}\right), 25.9\left(\mathrm{CH}_{3}\right), 26.3\left(\mathrm{CH}_{3}\right), 61.1,61.4(\mathrm{C} 1, \mathrm{C} 6)$, 74.5 (C2), 75.9 (C5), 77.8, 78.0 (C3, C4), $108.5\left(\underline{\mathrm{C}}\left(\mathrm{CH}_{3}\right)_{2}\right), 109.2\left(\underline{\mathrm{C}}\left(\mathrm{CH}_{3}\right)_{2}\right) ; \mathrm{m} / z(\mathrm{ESI}+\mathrm{ve}): 285\left([\mathrm{M}+\mathrm{Na}]^{+}\right.$, $100 \%$ ); HRMS $m / z$ (ESI+ve): found $285.1308[\mathrm{M}+\mathrm{Na}]^{+}, \mathrm{C}_{12} \mathrm{H}_{22} \mathrm{O}_{6} \mathrm{Na}^{+}$requires 285.1309.

\section{L-Glucitol [D-gulitol] 11}<smiles>OCC(O)C(O)C(O)CO</smiles>

DOWEX@ ${ }^{0}$ WX8-200 (5.00 g) was added to a solution of aldehyde $10(1.1 \mathrm{~g}, 3.85 \mathrm{mmol})$ in water (20 $\mathrm{mL}$ ). After stirring at room temperature for 24 hours, mass spectrum indicated that the reaction was complete and the resin was filtered off; the water was removed in vacuo to yield pure L-glucitol $\mathbf{1 1}$ as a colorless syrup further purification $(700 \mathrm{mg}, 100 \%)$ with identical NMR to those of a pure sample of L-glucitol.

1 Morimoto, K.; Shimonishi, T.; Miyake, S.; Takata, G.; Izumori, K. Biosci., Biotechnol., Biochem. 2013, 77, 253-258. 
$[\alpha]_{\mathrm{D}}{ }^{20}=+1.2\left(c, 0.75\right.$ in water); $\left[\right.$ lit. $^{2}[\alpha]_{\mathrm{D}}{ }^{29}=+1.7$ (c, 3.6 in water)]; $m / z$ (ESI+ve): $205\left([\mathrm{M}+\mathrm{Na}]^{+}\right.$, $100 \%$ ); HRMS $m / z$ (ESI+ve): found $205.0683[\mathrm{M}+\mathrm{Na}]^{+}, \mathrm{C}_{6} \mathrm{H}_{14} \mathrm{O}_{6} \mathrm{Na}^{+}$requires 205.0683.

Scheme 2: 6-Deoxy-D-gulonolactone 15, 6-Deoxy-D-gulose 18 and 6-Deoxy-D-gulitol [1-Deoxy-D-glucitol] 19

Methyl 6-deoxy-2,3:4,5-di-O-isopropylidene-6-iodo-D-gulonate 13<smiles>CC(=O)C1OC(C)(C)OC1C1OC(C)(C)OC1C</smiles>

Triflic anhydride (5.90 mL, $35.0 \mathrm{mmol})$ was added dropwise to a solution of 7 (7.8 g, $26.9 \mathrm{mmol})$ and anhydrous pyridine $(3.25 \mathrm{~mL}, 40.4 \mathrm{mmol})$ in dichloromethane $(50 \mathrm{~mL})$ at $-20{ }^{\circ} \mathrm{C}$. After stirring at $-20{ }^{\circ} \mathrm{C}$ for $3 \mathrm{~h}$, TLC (cyclohexane/ethyl acetate, 2:1) showed the formation of a major product $\left(\mathrm{R}_{\mathrm{f}} 0.68\right.$ ) and the disappearance of starting material $\left(R_{f} 0.28\right)$. The reaction mixture was diluted with dichloromethane $(10 \mathrm{~mL})$, washed with $\mathrm{HCl}\left(2 \mathrm{M}\right.$, aq. $60 \mathrm{~mL}$ ) and aqueous $\mathrm{NaHCO}_{3}$ (sat, $60 \mathrm{~mL}$ ). The organic layer was dried $\left(\mathrm{MgSO}_{4}\right)$ and the solvent was removed in vacuo to give a mixture of the crude triflate as a white solid (10.2 g). A solution of the crude triflate in butanone $(50 \mathrm{~mL})$ was treated with sodium iodide (8.1 g, $53.8 \mathrm{mmol}$ ); the flask was wrapped with aluminium foil and the reaction mixture stirred at $50{ }^{\circ} \mathrm{C}$ for 16 hours until mass spectrometry showed the formation of a product $([\mathrm{M}+\mathrm{H}]+401)$. After removal of solvent in vacuo, the residue was dissolve in cyclohexane $(80 \mathrm{~mL})$ and washed with water $(80 \mathrm{~mL})$. The aqueous layer was back extracted with cyclohexane $(2 \mathrm{x} 80 \mathrm{~mL})$. The organic layer was dried $\left(\mathrm{MgSO}_{4}\right)$, filtered and the solvent was removed to yield iodide $13(8.7 \mathrm{~g}, 81 \%)$ as a light yellow oil.

$[\alpha]_{\mathrm{D}^{20}}=+5.9\left(c, 1.08\right.$ in $\left.\mathrm{CHCl}_{3}\right) ; v_{\max }\left(\right.$ thin film): $1765(\mathrm{~s}, \mathrm{C}=0) ;{ }^{1} \mathrm{H} \mathrm{NMR}\left(\mathrm{CDCl}_{3}, 400 \mathrm{MHz}\right): 1.39(6 \mathrm{H}, \mathrm{s}, 2 \mathrm{x}$ $\left.\mathrm{CH}_{3}\right), 1.40\left(3 \mathrm{H}, \mathrm{s}, \mathrm{CH}_{3}\right), 1.61\left(3 \mathrm{H}, \mathrm{s}, \mathrm{CH}_{3}\right), 3.30\left(2 \mathrm{H}, \mathrm{d}, \mathrm{H} 6, J_{6,5} 5.5\right), 3.74\left(3 \mathrm{H}, \mathrm{s}, \mathrm{OCH}_{3}\right), 4.00\left(1 \mathrm{H}, \mathrm{dd}, \mathrm{H} 4, J_{4,3}\right.$ $\left.1.7, J_{4,5} 7.2\right), 4.16\left(1 \mathrm{H}, \mathrm{dt}, \mathrm{H} 5, J_{5,6}=J_{5,6^{\prime}} 5.5, J_{5,4} 7.1\right), 4.50\left(1 \mathrm{H}, \mathrm{dd}, \mathrm{H} 3, J_{3,4} 1.7, J_{3,2} 7.8\right), 4.70\left(1 \mathrm{H}, \mathrm{d}, \mathrm{H} 2, J_{2,3}\right.$ 7.8); ${ }^{13} \mathrm{C} \mathrm{NMR}\left(\mathrm{CDCl}_{3}, 100 \mathrm{MHz}\right): 5.7(\mathrm{C} 6), 25.5\left(\mathrm{CH}_{3}\right), 26.4\left(\mathrm{CH}_{3}\right), 26.7\left(\mathrm{CH}_{3}\right), 27.6\left(\mathrm{CH}_{3}\right), 52.2\left(\mathrm{OCH}_{3}\right)$, 75.2 (C2), 76.2 (C5), 76.6 (C3), 78.8 (C4), $110.2\left(\underline{\mathrm{C}}\left(\mathrm{CH}_{3}\right)_{2}\right), 111.1\left(\underline{\mathrm{C}}\left(\mathrm{CH}_{3}\right)_{2}\right), 170.3$ (C1); m/z (ESI+ve): $401\left([\mathrm{M}+\mathrm{H}]^{+}, 100 \%\right), 423\left([\mathrm{M}+\mathrm{Na}]^{+}, 15 \%\right) ; \mathrm{HRMS}(\mathrm{ESI}+\mathrm{ve})$ : found $423.0273[\mathrm{M}+\mathrm{Na}]^{+} ; \mathrm{C}_{13} \mathrm{H}_{21} \mathrm{NaO}_{6} \mathrm{I}^{+}$ requires 423.0275 .

\section{Methyl 6-deoxy-2,3:4,5-di-O-isopropylidene-D-gulonate 14}

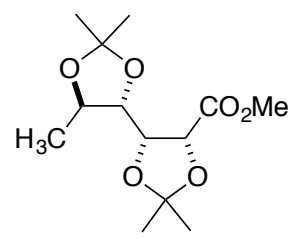

2 Wolfrom, M. L.; Lew, B. W.; Hales, R. A.; Goepp, R.; Max, Jr. J. Am. Chem. Soc., 1946, 68, 2342-2343. 
Palladium on charcoal (10 \% wt., $1.2 \mathrm{~g})$ and triethylamine $(4.2 \mathrm{~mL}, 30.0 \mathrm{mmol})$ were added to a solution of $13(12 \mathrm{~g}, 30.0 \mathrm{mmol})$ in methanol $(200 \mathrm{~mL})$. The reaction mixture was flushed with nitrogen, argon and hydrogen gas sequentially. Then the reaction mixture was stirred vigorously at room temperature under a hydrogen atmosphere for 20 hours until mass spectrometry showed the disappearance of starting material $([\mathrm{M}+\mathrm{H}]+401)$ and the formation of a product $([\mathrm{M}+\mathrm{Na}]+297)$. The reaction mixture was filtered and evaporated to dryness in vacuo, the residue was dissolved in ethyl acetate $(100 \mathrm{~mL})$ and washed with sodium thiosulfate (aq., sat, $100 \mathrm{~mL}$ ). Then the aqueous layer was back extracted with ethyl acetate $(2 \times 100 \mathrm{~mL})$ and the organic phase was dried $\left(\mathrm{MgSO}_{4}\right)$, filtered and solvent was removed in vacuo to yield the deoxy-gulonate $14(7.5 \mathrm{~g}, 91 \%)$ as a white crystalline solid.

m.p. $38-40{ }^{\circ} \mathrm{C} ;[\alpha]_{\mathrm{D}}{ }^{20}=+15.9\left(c, 1.38\right.$ in $\left.\mathrm{CHCl}_{3}\right) ; v_{\max }\left(\right.$ thin film): $1766(\mathrm{~s}, \mathrm{C}=0) ;{ }^{1} \mathrm{H} \mathrm{NMR}\left(\mathrm{CDCl}_{3}, 400\right.$ MHz): 1.30 (3H, d, H6, J6,5 6.1), 1.35 (6H, s, $2 \mathrm{x} \mathrm{CH}_{3}$ ), 1.39 (3H, s, $\left.\mathrm{CH}_{3}\right), 1.60$ (3H, s, $\left.\mathrm{CH}_{3}\right), 3.71$ (1H, dd, H4, $\left.J_{4,3} 1.8, J_{4,5} 8.2\right), 3.73\left(3 \mathrm{H}, \mathrm{s}, \mathrm{OCH}_{3}\right), 4.18\left(1 \mathrm{H}, \mathrm{dq}, \mathrm{H} 5, J_{5,6} 6.1, J_{5,4} 8.2\right), 4.34\left(1 \mathrm{H}, \mathrm{dd}, \mathrm{H} 3, J_{3,4} 1.8, J_{3,2} 7.8\right.$ ), $4.69\left(1 \mathrm{H}, \mathrm{d}, \mathrm{H} 2, \mathrm{~J}_{2,3} 7.8\right) ;{ }^{13} \mathrm{C} \mathrm{NMR}\left(\mathrm{CDCl}_{3}, 100 \mathrm{MHz}\right): 17.4(\mathrm{C} 6), 25.6\left(\mathrm{CH}_{3}\right), 26.4\left(\mathrm{CH}_{3}\right), 26.5\left(\mathrm{CH}_{3}\right), 27.5$ $\left(\mathrm{CH}_{3}\right), 52.1\left(\mathrm{OCH}_{3}\right), 73.0(\mathrm{C} 5), 75.4(\mathrm{C} 2, \mathrm{C} 3), 79.5(\mathrm{C} 4), 108.7\left(\underline{\mathrm{C}}\left(\mathrm{CH}_{3}\right)_{2}\right), 111.1\left(\underline{\mathrm{C}}\left(\mathrm{CH}_{3}\right)_{2}\right), 170.4(\mathrm{C} 1) ; \mathrm{m} / \mathrm{z}$ (ESI+ve): $297\left([\mathrm{M}+\mathrm{Na}]^{+}, 100 \%\right) ;$ HRMS (ESI+ve): found $297.1309[\mathrm{M}+\mathrm{Na}]^{+} ; \mathrm{C}_{13} \mathrm{H}_{22} \mathrm{NaO}_{6}{ }^{+}$requires 297.1309.

\section{6-Deoxy-D-gulono-1,4-lactone 15}<smiles>CC(O)C1OC(=O)[C@H](O)[C@@H]1O</smiles>

A solution of 14 (400 mg, $1.46 \mathrm{mmol}$ ) in trifluoroacetic acid/water (10 mL, 1:1) was stirred at room temperature for 16 hours until mass spectrometry showed the formation of desired lactone $\left([\mathrm{M}+\mathrm{Na}]^{+}\right.$ 185). The solvent was removed in vacuo to yield pure lactone 15 (236 mg, 100\%) as an off-white solid. m.p. $178-180{ }^{\circ} \mathrm{C} ;[\alpha]_{\mathrm{D}}{ }^{20}=-57\left(c, 0.89\right.$ in water) [lit. ${ }^{3}$ m.p. $180-181^{\circ} \mathrm{C} ;[\alpha]_{\mathrm{D}}{ }^{24}=-63.8(c, 0.88$ in water)]; $v_{\max }$ (thin film): 1748 (s, C=0); ${ }^{1} \mathrm{H}$ NMR ( $\mathrm{D}_{2} \mathrm{O}, 400 \mathrm{MHz}$ ): 1.28 (3H, d, H6, J6,5 6.4), $4.12\left(1 \mathrm{H}, \mathrm{dq}, \mathrm{H} 5, J_{5,6} 6.4\right.$, $\left.J_{5,4} 8.6\right), 4.31\left(1 \mathrm{H}, \mathrm{dd}, \mathrm{H} 4, J_{4,3} 2.7, J_{4,5} 8.6\right), 4.54\left(1 \mathrm{H}, \mathrm{dd}, \mathrm{H} 3, J_{3,4} 2.7, J_{3,2} 4.7\right), 4.74\left(1 \mathrm{H}, \mathrm{d}, \mathrm{H} 2, J_{2,3} 4.7\right) ;{ }^{33} \mathrm{C}$ NMR (D 20,100 MHz): 17.5 (C6), 66.5 (C5), 70.2 (C3), 71.6 (C2), 85.6 (C4), 178.8 (C1); m/z (ESI+ve): $185\left([\mathrm{M}+\mathrm{Na}]^{+}, 100 \%\right)$; HRMS (ESI+ve): found $185.0420[\mathrm{M}+\mathrm{Na}]^{+} ; \mathrm{C}_{6} \mathrm{H}_{10} \mathrm{NaO}_{5}{ }^{+}$requires 185.0420 .

6-Deoxy-2,3:4,5-di-O-isopropylidene-D-gulose

\section{6-Deoxy-2,3:4,5-di-O-isopropylidene-D-gulitol 16}<smiles>CC1OC(C)(C)O[C@H]1[C@H]1OC(C)(C)O[C@H]1CO</smiles>

16

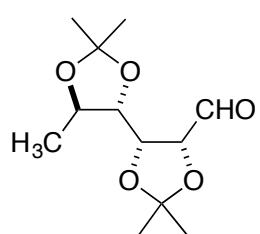

17

Diisobutylaluminum hydride (1.0 M in THF, $10.5 \mathrm{ml}, 10.5 \mathrm{mmol})$ was added dropwise to a solution of

3 Levene, P. A.; Compton, J. J. Biol. Chem. 1935, 111, 335-46. 
$14(1.16 \mathrm{~g}, 4.2 \mathrm{mmol})$ in dichloromethane $(20 \mathrm{~mL})$ at $-78^{\circ} \mathrm{C}$. The reaction mixture was stirred at $-78{ }^{\circ} \mathrm{C}$ for 2 hours until TLC (cyclohexane/ethyl acetate 2:1) indicated the formation of one major product $\left(\mathrm{R}_{\mathrm{f}}\right.$ $0.45)$ and one minor product $\left(\mathrm{R}_{\mathrm{f}} 0.30\right)$. Then the mixture was diluted with ethyl acetate $(10 \mathrm{~mL})$ and potassium sodium tartrate (sat, aq, $\sim 3 \mathrm{~mL}$ ) was added. After stirring for $10 \mathrm{~h}$, the mixture was diluted with water $(20 \mathrm{~mL})$ and extracted with ethyl acetate $(3 \times 20 \mathrm{~mL})$. The organic phase was dried $\left(\mathrm{MgSO}_{4}\right)$, filtered and the solvent was removed in vacuo to obtain a crude product that was purified by flash column chromatography to yield the protected aldehyde $\mathbf{1 7}$ as the major product (856 mg, 84\%), a colorless syrup and small amount of the protected alcohol 16 (150 mg, 14\%), a colorless syrup.

NMR data of 17: ${ }^{1} \mathrm{H}$ NMR ( $\left.\mathrm{CD}_{3} \mathrm{CN}, 400 \mathrm{MHz}\right): 1.26\left(3 \mathrm{H}, \mathrm{d}, \mathrm{H} 6, J_{6,5} 6.1\right), 1.31\left(3 \mathrm{H}, \mathrm{s}, \mathrm{CH}_{3}\right), 1.34\left(3 \mathrm{H}, \mathrm{s}, \mathrm{CH}_{3}\right)$, $1.38\left(3 \mathrm{H}, \mathrm{s}, \mathrm{CH}_{3}\right), 1.54\left(3 \mathrm{H}, \mathrm{s}, \mathrm{CH}_{3}\right), 3.63\left(1 \mathrm{H}, \mathrm{dd}, \mathrm{H} 4, J_{4,3} 1.0, J_{4,5} 8.4\right), 4.11\left(1 \mathrm{H}, \mathrm{dq}, \mathrm{H} 5, J_{5,6}=J_{5,6^{\prime}}=J_{5,6^{\prime \prime}} 6.1\right.$, $\left.J_{5,4} 8.4\right), 4.46\left(1 \mathrm{H}, \mathrm{dd}, \mathrm{H} 3, J_{3,4} 1.0, J_{3,2} 8.4\right), 4.49\left(1 \mathrm{H}, \mathrm{dd}, \mathrm{H} 2, J_{2,1} 2.0, J_{2,3} 8.4\right), 9.57\left(1 \mathrm{H}, \mathrm{d}, \mathrm{H} 1, J_{1,2} 2.0\right) ;{ }^{13} \mathrm{C}$ NMR (CD $\left.{ }_{3} \mathrm{CN}, 100 \mathrm{MHz}\right): 17.9(\mathrm{C} 6), 25.5\left(\mathrm{CH}_{3}\right), 26.8\left(\mathrm{CH}_{3}\right), 26.9\left(\mathrm{CH}_{3}\right), 27.8\left(\mathrm{CH}_{3}\right), 73.8$ (C5), 76.5 (C3), $80.3(\mathrm{C} 4), 82.0(\mathrm{C} 2), 109.5\left(\underline{\mathrm{C}}\left(\mathrm{CH}_{3}\right)_{2}\right), 111.6\left(\underline{\mathrm{C}}\left(\mathrm{CH}_{3}\right)_{2}\right), 201.7(\mathrm{C} 1)$.

16: $[\alpha]_{\mathrm{D}}{ }^{20}=+20.3\left(c, 0.6\right.$ in water); $v_{\max }$ (thin film): $3370(\mathrm{br}, \mathrm{OH}) ;{ }^{1} \mathrm{H}$ NMR $\left(\mathrm{CDCl}_{3}, 400 \mathrm{MHz}\right): 1.31(3 \mathrm{H}, \mathrm{d}$, $\mathrm{H} 6, J_{6,5}$ 6.1), 1.37 (3H, s, $\left.\mathrm{CH}_{3}\right), 1.41\left(3 \mathrm{H}, \mathrm{s}, \mathrm{CH}_{3}\right), 1.42\left(3 \mathrm{H}, \mathrm{s}, \mathrm{CH}_{3}\right), 1.51\left(3 \mathrm{H}, \mathrm{s}, \mathrm{CH}_{3}\right), 2.51(1 \mathrm{H}, \mathrm{br}-\mathrm{s}, \mathrm{OH})$, $3.59\left(1 \mathrm{H}, \mathrm{dd}, \mathrm{H} 4, J_{4,3} 1.5, J_{4,5} 8.6\right), 3.79\left(2 \mathrm{H}, \mathrm{H} 1, J_{1,2} 5.1\right), 4.08\left(1 \mathrm{H}, \mathrm{dd}, \mathrm{H} 3, J_{3,4} 1.5, J_{3,2} 6.7\right), 4.23$ (1H, dq, H5, $J_{5,6} 6.1, J_{5,4} 8.6$ ), 4.27 (1H, dd, H2, J2, 5.1, J2,3 6.7), 4.49 (1H, dd, H2, J2, 2.0, J2,3 8.4), 9.57 (1H, d, H1, J1,2 2.0); ${ }^{13} \mathrm{C} \mathrm{NMR}\left(\mathrm{CDCl}_{3}, 100 \mathrm{MHz}\right): 17.2(\mathrm{C} 6), 25.5\left(\mathrm{CH}_{3}\right), 26.3\left(\mathrm{CH}_{3}\right), 26.8\left(\mathrm{CH}_{3}\right), 27.5\left(\mathrm{CH}_{3}\right), 61.5(\mathrm{C} 1), 73.4$

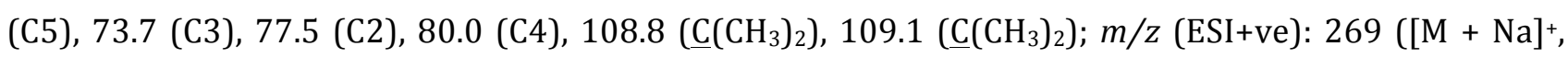
$100 \%)$; HRMS (ESI+ve): found 269.1359 [M+ Na] $; \mathrm{C}_{12} \mathrm{H}_{22} \mathrm{NaO}_{5}{ }^{+}$requires 269.1359.

\section{6-Deoxy-D-gulose 18}<smiles>CC(O)[C@H](O)C(O)C(O)C=O</smiles>

DOWEX@ 50WX8-200 (100 mg) was added to a solution of aldehyde 17 (856 mg, $3.51 \mathrm{mmol}$ ) in water/1,4-dioxane $(1: 1,10 \mathrm{~mL})$. The mixture was stirred at room temperature for 24 hours until mass spectrometry showed the formation of the desired product $\left([\mathrm{M}+\mathrm{Na}]^{+} 187\right)$. Then resin was filtered off and water was removed in vacuo to yield 6-deoxy-D-gulose 18 (570 mg, 99\%) as a colorless syrup ( $\alpha / \beta$ pyranosides ratio $1: 10)$.

$[\alpha]_{\mathrm{D}^{20}}=-20.0\left(c, 1.0\right.$ in water), [lit. ${ }^{4}[\alpha]_{\mathrm{D}}{ }^{20}=-39.3\left(c, 1.0\right.$ in water); lit. $^{7}[\alpha]_{\mathrm{D}}{ }^{20}=-20.3(c, 0.6$ in water $\left.)\right]$; $v_{\max }$ (thin film): 3440 (br, OH); NMR data for major $\beta$ anomer: ${ }^{1} \mathrm{H}$ NMR $\left(\mathrm{D}_{2} \mathrm{O}, 400 \mathrm{MHz}\right): 1.22$ (3H, d, H6, $J_{6,5} 6.6$ ), $3.57\left(1 \mathrm{H}, \mathrm{dd}, \mathrm{H} 2, J_{2,3} 3.4, J_{2,1} 8.4\right.$ ), $3.62\left(1 \mathrm{H}, \mathrm{dd}, \mathrm{H} 4, J_{4,5} 0.8, J_{4,3} 3.5\right), 4.07\left(1 \mathrm{H}, \mathrm{t}, \mathrm{H} 3, J_{3,4}=J_{3,2} 3.5\right.$ ), 4.10 (1H, dq, H5, J5,4 0.8, J5,6 6.6), 4.85 (1H, d, H1, J1,2 8.4); ${ }^{13} \mathrm{C}$ NMR ( $\left.{ }_{2} \mathrm{O}, 100 \mathrm{MHz}\right): 15.7$ (C6), 69.5 (C2), 69.9 (C5), 71.8 (C3), 72.5 (C4), 94.2 (C1); m/z (ESI+ve): 187 ([M + Na]+, 100\%); HRMS (ESI+ve): found 187.0575 $[\mathrm{M}+\mathrm{Na}]^{+} ; \mathrm{C}_{6} \mathrm{H}_{12} \mathrm{NaO}_{5}{ }^{+}$requires 187.0577.

\section{6-Deoxy-D-gulitol [1-deoxy-L-glucitol] 19}

4 Kaufmann, H. Helv. Chim. Acta. 1965, 48, 769-79. 


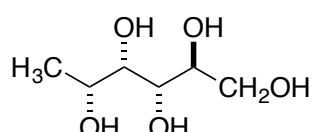

DOWEX@ 50WX8-200 (30 mg) was added into a solution of 16 (150 mg, $0.61 \mathrm{mmol})$ was dissolved into water/1,4-dioxane $(1: 1,10 \mathrm{~mL})$. The mixture was stirred at room temperature for $24 \mathrm{~h}$ until mass spectrometry showed the formation of desired product $([\mathrm{M}+\mathrm{Na}]+187)$. Then resin was filtered off and water was removed in vacuo to yield 6-deoxy-D-gulitol 19 (102 mg, 100\%) as a colorless syrup.

$[\alpha]_{\mathrm{D}}{ }^{20}=-2.8\left(c, 1.4\right.$, water); [Lit. ${ }^{5}[\alpha]_{\mathrm{D}^{20}}=-3.9$ (no $c$ and temperature indicated, water)]; ${ }^{1} \mathrm{H}$ NMR $\left(\mathrm{D}_{2} \mathrm{O}\right.$, $400 \mathrm{MHz}$ ): 1.19 (3H, d, H6, J6,5 6.4), $3.61\left(1 \mathrm{H}, \mathrm{t}, \mathrm{H} 4, J_{4,3}=J_{4,5} 6.4\right.$ ), $3.62(1 \mathrm{H}, \mathrm{m}, \mathrm{H} 3), 3.64$ (1H, dd, H1, J1,2, 6.3, Jgem 11.8), 4.76 (1H, ddd, H2, J2,1' 2.9, J2,1 6.3, J2,3 8.7), 4.83 (1H, dd, H1', J',2, 2.9, Jgem 11.8), 3.90 (1H, quint, $\mathrm{H} 5, J_{5,4}=J_{5,6} 6.4$ ); ${ }^{13} \mathrm{C}$ NMR ( ${ }_{2} \mathrm{O}, 100 \mathrm{MHz}$ ): 18.8 (C6), 63.5 (C1), 69.4 (C5), 71.3 (C3), 71.5 (C2), 74.4 (C4); $m / z$ (ESI+ve): 189 ([M + Na] $\left.]^{+}, 100 \%\right) ;$ HRMS (ESI+ve): found $189.0731[\mathrm{M}+\mathrm{Na}]^{+} ; \mathrm{C}_{6} \mathrm{H}_{14} \mathrm{NaO}_{5}{ }^{+}$ requires 189.0733 .

\section{Scheme 3: meso-D-glycero-D-gulo-Heptitol 22, 6-Deoxy-L-glucose [L-quinovose] 31 and}

\section{6-Deoxy-L-glucitol 33}

\section{2,3:4,5:6,7-Tri-O-isopropylidene-D-glycero-D-gulo-heptitol 20}<smiles>CC1(C)OCC(C2OC(C)(C)OC2C2OC(C)(C)OC2CO)O1</smiles>

Lithium aluminum hydride solution (1 M in THF, $19.4 \mathrm{~mL}, 19.4 \mathrm{mmol}$ ) was added dropwise to a stirred solution of $2(7.0 \mathrm{~g}, 19.4 \mathrm{mmol})$ in THF $(40 \mathrm{~mL})$ at $0{ }^{\circ} \mathrm{C}$. The reaction mixture was refluxed for 1 hour after which TLC analysis (cyclohexane/ethyl acetate, 2:1) showed formation of a single product ( $\mathrm{R}_{\mathrm{f}}$ $0.33)$. The excess hydride was quenched by dropwise addition of $\mathrm{NH}_{4} \mathrm{Cl}(\sim 5 \mathrm{~mL}$, sat. aq. $)$ at $0{ }^{\circ} \mathrm{C}$ and the resulting mixture was dried $\left(\mathrm{MgSO}_{4}\right)$, filtered (eluting with ethyl acetate) and concentrated in vacuo to give a residue that was dissolved in ethyl acetate $(50 \mathrm{~mL})$ and washed with water $(2 \times 50 \mathrm{~mL})$. The organic phase was dried $\left(\mathrm{MgSO}_{4}\right)$, filtered and solvent was removed in vacuo to give pure product 20 $(4.6 \mathrm{~g}, 72 \%)$ as a clear oil.

$[\alpha]_{\mathrm{D}}{ }^{20}=+33(c, 0.83$ in $\mathrm{MeOH}) ; v_{\max }\left(\right.$ thin film): 3300 (br, OH); ${ }^{1} \mathrm{H}$ NMR $\left(\mathrm{CDCl}_{3}, 400 \mathrm{MHz}\right): 1.34(3 \mathrm{H}, \mathrm{s}$, $\left.\mathrm{CH}_{3}\right), 1.35\left(3 \mathrm{H}, \mathrm{s}, \mathrm{CH}_{3}\right), 1.39\left(3 \mathrm{H}, \mathrm{s}, \mathrm{CH}_{3}\right), 1.42\left(3 \mathrm{H}, \mathrm{s}, \mathrm{CH}_{3}\right), 1.43\left(3 \mathrm{H}, \mathrm{s}, \mathrm{CH}_{3}\right), 1.50\left(3 \mathrm{H}, \mathrm{s}, \mathrm{CH}_{3}\right), 1.85(1 \mathrm{H}$,

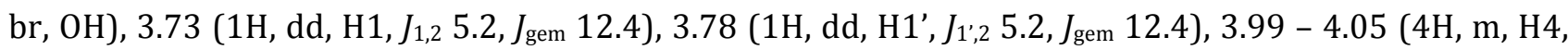
H5, H6, H7), 4.12 - 4.15 (1H, m, H7'), $4.25\left(1 \mathrm{H}, \mathrm{dt}, \mathrm{H} 2, J_{2,1}=J_{2,1^{\prime}} 5.2, J_{2,3} 6.6\right), 4.33\left(1 \mathrm{H}, \mathrm{br}-\mathrm{d}, \mathrm{H} 3, J_{3,2} 6.6\right.$ ); ${ }^{13} \mathrm{C}$ NMR $\left(\mathrm{CDCl}_{3}, 100 \mathrm{MHz}\right): 25.3\left(\mathrm{CH}_{3}\right), 25.5\left(\mathrm{CH}_{3}\right), 26.4\left(\mathrm{CH}_{3}\right), 26.8\left(\mathrm{CH}_{3}\right), 27.0\left(\mathrm{CH}_{3}\right), 27.3\left(\mathrm{CH}_{3}\right), 61.8$ (C1), 67.9 (C7), 75.0 (C3), 77.4 (C2), 77.5, 77.7, 78.0 (C4, C5, C6), $108.4\left(\underline{C}\left(\mathrm{CH}_{3}\right)_{2}\right), 109.6\left(\underline{\mathrm{C}}\left(\mathrm{CH}_{3}\right)_{2}\right)$, 
$110.1\left(\underline{\mathrm{C}}\left(\mathrm{CH}_{3}\right)_{2}\right) ; \mathrm{m} / z$ (ESI+ve): $355\left([\mathrm{M}+\mathrm{Na}]^{+}, 100 \%\right) ;$ HRMS (ESI+ve): Found $355.1726\left([\mathrm{M}+\mathrm{Na}]^{+}\right)$; $\mathrm{C}_{16} \mathrm{H}_{28} \mathrm{NaO}_{7}+$ requires 355.1727 .

\section{meso-D-glycero-D-gulo-Heptitol 21}<smiles>OCC(O)C(O)C(O)C(O)CO</smiles>

DOWEX $^{\circledR}$ 50WX8-200 ( $\sim 300 \mathrm{mg}$, pre-washed with water) was added into a solution of 20 (1.3 g, 3.9 mmol) in water/1,4-dioxane $(30 \mathrm{~mL}, 1: 1)$. After stirring at room temperature for $24 \mathrm{~h}$, TLC (cyclohexane/ethyl acetate, 2:1) showed the disappearance of starting material $\left(\mathrm{R}_{\mathrm{f}} 0.33\right)$. After filtration, solvent was removed in vacuo to obtain unprotected heptitol 21 (780 mg, 94\%) as a white solid. ${ }^{13} \mathrm{C}$ spectrum fitted previous report. ${ }^{6}$

m.p.: $120-122{ }^{\circ} \mathrm{C}$ [lit. 7 m.p.: $134^{\circ} \mathrm{C}$; lit. ${ }^{8}$ m.p.: $\left.134-135^{\circ} \mathrm{C}\right] ;[\alpha]_{\mathrm{D}^{20}}=0\left(c, 1.0\right.$ in water); $v_{\max }($ thin film): 3370 (br, OH); ${ }^{1} \mathrm{H}$ NMR ( $\left.{ }_{2} \mathrm{O}, 400 \mathrm{MHz}\right): 3.62$ - 3.67 (2H, m, H1, H7), 3.74 (2H, dd, H3(H5), J 2.9, J 7.6), 3.76 - $3.82\left(4 \mathrm{H}, \mathrm{m}, \mathrm{H} 1^{\prime}, \mathrm{H} 2, \mathrm{H} 6, \mathrm{H7}\right.$ ), 4.01 (1H, t, H4, J4,3 = J4,5 2.9); ${ }^{13} \mathrm{C}$ NMR (D $\left.{ }_{2} \mathrm{O}, 100 \mathrm{MHz}\right): 63.2$ (C1/C7), 69.0 (C4), 74.7 (C2/C6), 73.5 (C3/C5); m/z (ESI+ve): 235 ([M + Na]+, 100\%); HRMS (ESI+ve): found 235.0789 [M+ Na]+; $\mathrm{C}_{7} \mathrm{H}_{16} \mathrm{NaO}_{7}+$ requires 235.0788.

\section{1-O-Mesyl-2,3:4,5:6,7-tri-O-isopropylidene-D-glycero-D-gulo-heptitol 23}<smiles>COOCC1OC(C)(C)OC1C1OC(C)(C)OC1C1COC(C)(C)O1</smiles>

Mesyl chloride $(0.014 \mathrm{~mL}, 0.18 \mathrm{mmol})$ and triethylamine $(0.06 \mathrm{~mL}, 0.45 \mathrm{mmol})$ were added to a solution of 20 (50 mg, $0.15 \mathrm{mmol})$ in DCM $(2 \mathrm{~mL})$ at $0{ }^{\circ} \mathrm{C}$. The reaction mixture was stirred at room temperature for 12 hours when TLC (cyclohexane/ethyl acetate 2:1) showed the formation of one product $\left(R_{f} 0.65\right)$ and the consumption of starting material $\left(R_{f} 0.33\right)$. The reaction mixture was diluted with DCM $(8 \mathrm{~mL})$ and washed with distilled water $(10 \mathrm{~mL})$. The organic phase was dried $\left(\mathrm{MgSO}_{4}\right)$, filtered and solvent was removed in vacuo to yield a residue that was purified by flash column chromatography (cyclohexane/ethyl acetate 5:1) to yield mesylate $\mathbf{2 3}$ as a white solid (50 mg, 82\%). m.p. $76-78{ }^{\circ} \mathrm{C} ;[\alpha]_{\mathrm{D}^{20}}=+9.9\left(c, 1.08, \mathrm{CH}_{3} \mathrm{CN}\right) ; v_{\max }$ (thin film): fingerprint region only; ${ }^{1} \mathrm{H} \mathrm{NMR}\left(\mathrm{CD}_{3} \mathrm{CN}\right.$, $500 \mathrm{MHz}$ ): $1.31\left(3 \mathrm{H}, \mathrm{s}, \mathrm{CH}_{3}\right), 1.35\left(3 \mathrm{H}, \mathrm{s}, \mathrm{CH}_{3}\right), 1.36\left(3 \mathrm{H}, \mathrm{s}, \mathrm{CH}_{3}\right), 1.39\left(6 \mathrm{H}, \mathrm{s}, 2 \times \mathrm{CH}_{3}\right), 1.48\left(3 \mathrm{H}, \mathrm{s}, \mathrm{CH}_{3}\right)$, $3.08\left(3 \mathrm{H}, \mathrm{s}, \mathrm{SO}_{2} \mathrm{CH}_{3}\right), 3.90\left(1 \mathrm{H}, \mathrm{dd}, \mathrm{H} 7, J_{7,6} 4.1, J_{\text {gem }} 11.0\right), 3.92-3.94(1 \mathrm{H}, \mathrm{m}, \mathrm{H} 6), 3.97\left(1 \mathrm{H}, \mathrm{dd}, \mathrm{H} 4, J_{4,3} 1.3\right.$, $J_{4,5} 7.9$ ), $4.07-4.09$ (1H, m, H5), 4.39 (1H, dd, H1, J1,2 8.2, Jgem 10.7), 4.41 (1H, dd, H3, J3,4 1.3, J3,2 7.2), $4.45\left(1 \mathrm{H}, \mathrm{dd}, \mathrm{H} 1^{\prime}, J_{1^{\prime}, 2} 3.5\right.$, Jgem 10.7$), 4.53\left(1 \mathrm{H}, \mathrm{H} 2\right.$, ddd, $\left.J_{2,1^{\prime}} 3.5, J_{2,3} 7.2, J_{2,1} 8.2\right) ;{ }^{13} \mathrm{C}$ NMR $\left(\mathrm{CD}_{3} \mathrm{CN}, 100\right.$ MHz): $25.5\left(\mathrm{CH}_{3}\right), 25.6\left(\mathrm{CH}_{3}\right), 27.08\left(\mathrm{CH}_{3}\right), 27.11\left(\mathrm{CH}_{3}\right), 27.2\left(\mathrm{CH}_{3}\right), 27.6\left(\mathrm{CH}_{3}\right), 37.7\left(\mathrm{SO}_{2} \mathrm{CH}_{3}\right), 68.2(\mathrm{C} 7)$, 
71.3 (C1), 75.7 (C3), 76.3 (C2), 77.9 (C5), 78.5 (C6), 78.9 (C4), $110.0\left(\underline{\mathrm{C}}\left(\mathrm{CH}_{3}\right)_{2}\right), 110.5\left(\underline{\mathrm{C}}\left(\mathrm{CH}_{3}\right)_{2}\right), 110.7$

$\left(\underline{\mathrm{C}}\left(\mathrm{CH}_{3}\right)_{2}\right) ; \mathrm{m} / z$ (ESI+ve): $433\left([\mathrm{M}+\mathrm{Na}]^{+}, 100 \%\right) ;$ HRMS (ESI+ve): found $433.1497[\mathrm{M}+\mathrm{Na}]^{+}$; $\mathrm{C}_{16} \mathrm{H}_{28} \mathrm{NaO}_{7}{ }^{+}$requires 433.1503 .

1,2:4,5:6,7-Tri-O-isopropylidene-D-glycero-D-gulo-heptitol

and

1-Deoxy-1-iodo-2,3:4,5:6,7-tri-O-isopropylidene-D-glycero-D-gulo-heptitol 25

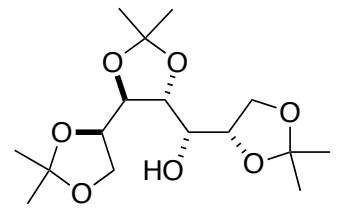

26<smiles>CC1OC(C)(C)O[C@H]1C1OC(C)(C)OC1C1COC(C)(C)O1</smiles>

25

Imidazole (16 mg, $0.23 \mathrm{mmol}$ ), triphenylphosphine (60 mg, $0.23 \mathrm{mmol}$ ) and iodine (58 $\mathrm{mg}, 0.23 \mathrm{mmol}$ ) were added into a solution of 20 (50 mg, $0.15 \mathrm{mmol})$ in toluene ( $3 \mathrm{~mL})$. After stirring at $80{ }^{\circ} \mathrm{C}$ for 2 hours, TLC (cyclohexane/ethyl acetate, $2: 1$ ) showed the formation of two products $\left(R_{f} 0.56,0.42\right)$ and some remaining starting material $\left(\mathrm{R}_{\mathrm{f}} 0.33\right)$. The reaction mixture was stirred for a further of 2 hours until all starting material disappeared. The solvent was removed in vacuo and the residue was purified by flash column chromatography (cyclohexane/ethyl acetate, 5:1 $\rightarrow$ 1:1) to give the iodide 25 (32 mg, 48\%) and secondary alcohol 26 (20 mg, 37\%).

Full data for iodide $\mathbf{2 5}$ is available in the following experimental.

26: $[\alpha]_{\mathrm{D}}{ }^{20}=+3.9(c, 1.2$ in $\mathrm{MeOH}) ; v_{\max }$ (thin film): 3400 (br, OH); ${ }^{1} \mathrm{H} \mathrm{NMR}\left(\mathrm{CDCl}_{3}, 400 \mathrm{MHz}\right): 1.32(3 \mathrm{H}, \mathrm{s}$, $\left.\mathrm{CH}_{3}\right), 1.36\left(3 \mathrm{H}, \mathrm{s}, \mathrm{CH}_{3}\right), 1.39\left(3 \mathrm{H}, \mathrm{s}, \mathrm{CH}_{3}\right), 1.40\left(3 \mathrm{H}, \mathrm{s}, \mathrm{CH}_{3}\right), 1.41\left(3 \mathrm{H}, \mathrm{s}, \mathrm{CH}_{3}\right), 1.43\left(3 \mathrm{H}, \mathrm{s}, \mathrm{CH}_{3}\right), 2.17(1 \mathrm{H}, \mathrm{d}$, $\left.\mathrm{OH}, J_{\mathrm{OH}, 3} 9.2\right), 3.68\left(1 \mathrm{H}, \mathrm{dt}, \mathrm{H} 3, J_{3,4} 0.9, J_{3, \mathrm{OH}}=J_{3,2} 9.2\right.$ ) $) 3.93\left(1 \mathrm{H}, \mathrm{dd}, \mathrm{H} 7, J_{7,6} 5.2, J_{\mathrm{gem}} 8.4\right), 3.95$ (1H, t, H5, $J_{5,4}=J_{5,6} 8.0$ ), $4.03-4.09\left(4 \mathrm{H}, \mathrm{m}, \mathrm{H} 2, \mathrm{H} 6, \mathrm{H} 1, \mathrm{H} 1^{\prime}\right), 4.15$ (1H, dd, H7', J7,6 6.0, Jgem 8.4), 4.20 (1H, dd, H4, J4,3 0.9, J4,5 8.0); ${ }^{13} \mathrm{C} \mathrm{NMR}\left(\mathrm{CDCl}_{3}, 100 \mathrm{MHz}\right): 25.3\left(\mathrm{CH}_{3}\right), 25.4\left(\mathrm{CH}_{3}\right), 26.7\left(\mathrm{CH}_{3}\right), 26.8\left(\mathrm{CH}_{3}\right), 27.0\left(\mathrm{CH}_{3}\right), 27.2$ $\left(\mathrm{CH}_{3}\right), 66.6$ (C7), 67.9 (C1), 70.0 (C3), 76.9 (C2, C5, C6), 79.1 (C4), $109.3\left(\underline{\mathrm{C}}\left(\mathrm{CH}_{3}\right)_{2}\right), 109.7\left(\underline{\mathrm{C}}\left(\mathrm{CH}_{3}\right)_{2}\right)$, $109.8\left(\underline{\mathrm{C}}\left(\mathrm{CH}_{3}\right)_{2}\right) ; \mathrm{m} / z(\mathrm{ESI}+\mathrm{ve}): 355\left([\mathrm{M}+\mathrm{Na}]^{+}, 100 \%\right) ;$ HRMS (ESI+ve): found $355.1731[\mathrm{M}+\mathrm{Na}]^{+}$; $\mathrm{C}_{16} \mathrm{H}_{28} \mathrm{NaO}_{7}+$ requires 355.1727 .

\section{1-Deoxy-1-iodide-2,3:4,5:6,7-tri-O-isopropylidene-D-glycero-D-gulo-heptitol 25}<smiles>CC1OC(C)(C)OC1[C@H]1OC(C)(C)OC1C1COC(C)(C)O1</smiles>

Imidazole (13.1 g, $192.6 \mathrm{mmol})$, triphenylphosphine (12.1 g, $46.1 \mathrm{mmol})$ and iodine (11.7 g, $46.2 \mathrm{mmol})$ were added to a solution of $20(12.0 \mathrm{~g}, 36.1 \mathrm{mmol})$ in toluene $(250 \mathrm{~mL})$. After stirring at $80{ }^{\circ} \mathrm{C}$ for 2 hours, TLC (cyclohexane/ethyl acetate, 2:1) showed the formation of one major product $\left(\mathrm{R}_{\mathrm{f}} 0.78\right)$ and the disappearance of starting material $\left(\mathrm{R}_{\mathrm{f}} 0.47\right)$. The solvent was removed in vacuo and the residue was stirred with hexane $(100 \mathrm{~mL})$ for $5 \mathrm{~min}$. Silica gel $(\sim 12 \mathrm{~g})$ was added and the silica gel was washed with 
hexane ( $3 \times 100 \mathrm{~mL}$ ). After removing hexane in vacuo, pure iodide 25 was obtained as a colorless oil $(11.0 \mathrm{~g}, 69 \%)$.

$[\alpha]_{\mathrm{D}^{20}}=+11.1(c, 1.24$ in $\mathrm{MeOH}) ; v_{\max }\left(\right.$ thin film): fingerprint region only; ${ }^{1} \mathrm{H}$ NMR $\left(\mathrm{CDCl}_{3}, 400 \mathrm{MHz}\right): 1.34$ $\left(3 \mathrm{H}, \mathrm{s}, \mathrm{CH}_{3}\right), 1.36\left(3 \mathrm{H}, \mathrm{s}, \mathrm{CH}_{3}\right), 1.37\left(3 \mathrm{H}, \mathrm{s}, \mathrm{CH}_{3}\right), 1.41\left(3 \mathrm{H}, \mathrm{s}, \mathrm{CH}_{3}\right), 1.42\left(3 \mathrm{H}, \mathrm{s}, \mathrm{CH}_{3}\right), 1.50\left(3 \mathrm{H}, \mathrm{s}, \mathrm{CH}_{3}\right)$, $3.39\left(1 \mathrm{H}, \mathrm{dd}, \mathrm{H} 1, J_{1,2} 8.2\right.$, Jgem 10.0), 3.48 (1H, dd, H1', J',2 5.5, Jgem 10.0), 3.92 (1H, a-t, H5, J5,4 $=J_{5,6} 8.1$ ), 3.98 (1H, dd, H7, J7,6 4.5, Jgem 8.5), $4.02-4.07$ (2H, m, H4, H6), 4.14 (1H, dd, H7', J $7^{\prime}, 6$ 6.0, Jgem 8.5), 4.31 $\left(1 \mathrm{H}, \mathrm{dd}, \mathrm{H} 3, J_{3,4} 2.0, J_{3,2} 6.0\right), 4.51\left(1 \mathrm{H}, \mathrm{ddd}, \mathrm{H} 2, J_{2,1^{\prime}} 5.5, J_{2,3} 6.0, J_{2,1} 8.2\right) ;{ }^{13} \mathrm{C} \mathrm{NMR}\left(\mathrm{CDCl}_{3}, 100 \mathrm{MHz}\right): 4.27$ (C1), $25.3\left(\mathrm{CH}_{3}\right), 25.4\left(\mathrm{CH}_{3}\right), 26.7\left(\mathrm{CH}_{3}\right), 26.8\left(\mathrm{CH}_{3}\right), 27.1\left(\mathrm{CH}_{3}\right), 27.3\left(\mathrm{CH}_{3}\right), 67.9(\mathrm{C} 7), 76.0(\mathrm{C} 3), 77.2$ (C6), 77.7 (C5), 78.3 (C2, C4), $108.8\left(\underline{\mathrm{C}}\left(\mathrm{CH}_{3}\right)_{2}\right), 109.8\left(\underline{\mathrm{C}}\left(\mathrm{CH}_{3}\right)_{2}\right), 110.0\left(\underline{\mathrm{C}}\left(\mathrm{CH}_{3}\right)_{2}\right) ; \mathrm{m} / z$ (ESI+ve): $355([\mathrm{M}$ $+\mathrm{Na}]^{+}, 100 \%$ ); HRMS (ESI+ve): found $465.0745[\mathrm{M}+\mathrm{Na}]^{+} ; \mathrm{C}_{16} \mathrm{H}_{27} \mathrm{NaO}_{6} \mathrm{I}^{+}$requires 465.0745.

\section{1-Deoxy-2,3:4,5:6,7-tri-O-isopropylidene-D-glycero-D-gulo-heptitol 27}<smiles>CC1OC(C)(C)OC1C1OC(C)(C)OC1C1COC(C)(C)O1</smiles>

Palladium black (10\% wt., $30 \mathrm{mg}$ ) and triethylamine $(0.09 \mathrm{~mL}, 0.68 \mathrm{mmol})$ was added to a solution of 25 (300 mg, $0.68 \mathrm{mmol})$ in methanol $(10 \mathrm{~mL})$. The reaction mixture was flushed with nitrogen, argon and hydrogen gas sequentially. Then the reaction mixture was stirred vigorously at room temperature under a hydrogen atmosphere for $24 \mathrm{~h}$ until TLC (cyclohexane/ethyl acetate 1:1) showed the formation of one product $\left(R_{f} 0.74\right)$ and the disappearance of starting material $\left(R_{f} 0.78\right)$. The reaction mixture was filtered and evaporated to dryness in vacuo; the residue was dissolved in cyclohexane $(10 \mathrm{~mL})$ and washed with water (aq., sat, $20 \mathrm{~mL}$ ). Then the aqueous layer was back extracted with cyclohexane $(2 \mathrm{x}$ $10 \mathrm{~mL}$ ). The organic phase was dried $\left(\mathrm{MgSO}_{4}\right)$, filtered and solvent was removed in vacuo to yield triacetonide 27 (172 $\mathrm{mg}, 80 \%$ ) as a light yellow oil.

$[\alpha]_{\mathrm{D}}{ }^{20}=+33.3\left(c, 1.0\right.$ in $\left.\mathrm{CHCl}_{3}\right) ; v_{\max }$ (thin film): fingerprint region only; ${ }^{1} \mathrm{H} \mathrm{NMR}\left(\mathrm{CDCl}_{3}, 400 \mathrm{MHz}\right): 1.34$ $\left(3 \mathrm{H}, \mathrm{s}, \mathrm{CH}_{3}\right), 1.35\left(3 \mathrm{H}, \mathrm{s}, \mathrm{CH}_{3}\right), 1.36\left(3 \mathrm{H}, \mathrm{s}, \mathrm{CH}_{3}\right), 1.41\left(3 \mathrm{H}, \mathrm{d}, \mathrm{H} 1, J_{1,2} 6.5\right), 1.43\left(6 \mathrm{H}, \mathrm{s}, 2 \mathrm{x} \mathrm{CH}_{3}\right), 1.52(3 \mathrm{H}, \mathrm{s}$, $\mathrm{CH}_{3}$ ), $3.85\left(1 \mathrm{H}, \mathrm{dd}, \mathrm{H} 4, J_{4,3}\right.$ 1.4, J4,5 7.9), $3.90\left(1 \mathrm{H}, \mathrm{t}, \mathrm{H} 5, J_{5,6}=J_{5,4} 8.0\right), 3.97$ (1H, dd, H7, J7,6 4.9, Jgem 8.4), 4.03 (1H, ddd, H6, J6,7 4.9, J6,7 6.0, J6,5 8.0), 4.13 (1H, dd, H7', J7,6 6.0, Jgem 8.4), 4.20 (1H, dd, H3, J3,4 1.4, J3,2 6.6), $4.40\left(1 \mathrm{H}\right.$, quint, $\left.\mathrm{H} 2, J_{2,3}=J_{2,1} 6.4\right)$; ${ }^{13} \mathrm{C} \mathrm{NMR}\left(\mathrm{CDCl}_{3}, 100 \mathrm{MHz}\right): 15.2(\mathrm{C} 1), 25.4\left(\mathrm{CH}_{3}\right), 25.5\left(\mathrm{CH}_{3}\right), 26.7$ $\left(\mathrm{CH}_{3}\right), 26.8\left(\mathrm{CH}_{3}\right), 27.0\left(\mathrm{CH}_{3}\right), 27.2\left(\mathrm{CH}_{3}\right), 68.1(\mathrm{C} 7), 72.9$ (C2), 76.2 (C3), 77.4, 77.5 (C5, C6), $79.1(\mathrm{C} 4)$, $108.0\left(\underline{\mathrm{C}}\left(\mathrm{CH}_{3}\right)_{2}\right), 109.6\left(\underline{\mathrm{C}}\left(\mathrm{CH}_{3}\right)_{2}\right), 109.7\left(\underline{\mathrm{C}}\left(\mathrm{CH}_{3}\right)_{2}\right) ; \mathrm{m} / z$ (ESI+ve): $339\left([\mathrm{M}+\mathrm{Na}]^{+}, 100 \%\right) ; \mathrm{HRMS}$ (ESI+ve): found $339.1778[\mathrm{M}+\mathrm{Na}]+; \mathrm{C}_{16} \mathrm{H}_{28} \mathrm{NaO}_{6}{ }^{+}$requires 339.1778.

\section{1-Deoxy-2,3:4,5-di-O-isopropylidene-1-iodo-D-glycero-D-gulo-heptitol 28}


<smiles>CC1OC(C)(C)OC1[C@H]1OC(C)(C)OC1C(O)CO</smiles>

A solution of the triacetonide 25 (9.0 g, $20.4 \mathrm{mmol})$ in acetic acid/water (7:3, $50 \mathrm{~mL})$ was stirred at room temperature for 16 hours when TLC (cyclohexane/ethyl acetate, 1:1) showed the formation of a new spot $\left(\mathrm{R}_{\mathrm{f}} 0.34\right)$ and some starting material $\left(\mathrm{R}_{\mathrm{f}} 0.78\right)$. Then solvent was removed in vacuo to obtain a residue that was mixed with water $(70 \mathrm{~mL})$. Cyclohexane $(3 \times 70 \mathrm{~mL})$ was used to extract the remaining starting material 25. Then the aqueous layer was extracted with dichloromethane ( $3 \times 70 \mathrm{~mL})$. After removing dichloromethane in vacuo, pure diol 28 (4.0 g, 49\%) was obtained as a colorless oil. The remaining starting material $25(\sim 3.0 \mathrm{~g})$ from the cyclohexane phase was recycled and used to repeat the procedure above to obtain more diol 28 (6.0 g in total, 74\%);

$[\alpha]_{\mathrm{D}}{ }^{20}=+21.6(c, 1.15$ in $\mathrm{MeOH}) ; v_{\max }$ (thin film): $3380(\mathrm{br}, \mathrm{OH}) ;{ }^{1} \mathrm{H}$ NMR $\left(\mathrm{CD}_{3} \mathrm{CN}, 400 \mathrm{MHz}\right): 1.34(3 \mathrm{H}, \mathrm{s}$, $\left.\mathrm{CH}_{3}\right), 1.35\left(3 \mathrm{H}, \mathrm{s}, \mathrm{CH}_{3}\right), 1.38\left(3 \mathrm{H}, \mathrm{s}, \mathrm{CH}_{3}\right), 1.46\left(3 \mathrm{H}, \mathrm{s}, \mathrm{CH}_{3}\right), 2.85\left(1 \mathrm{H}, \mathrm{t}, \mathrm{OH}-7, J_{\mathrm{OH}, 7} 5.6\right), 3.22(1 \mathrm{H}, \mathrm{d}, \mathrm{OH}-6$, $J_{\mathrm{OH}, 6} 5.4$ ), 3.42 (1H, dd, H1, J1,2 9.0, Jgem 10.3 ), 3.49 (1H, a-ddd, H7, J7, Он 5.4, J7,6 5.6, Jgem 10.8), 3.54 (1H, dd, H1', J1,2 4.9, Jgem 10.3), $3.57-3.67$ (2H, m, H6, H7'), 3.87 (1H, t, H5, J5,4 $=J_{5,6} 7.8$ ), $4.10\left(1 \mathrm{H}, \mathrm{dd}, \mathrm{H} 4, J_{4,3} 1.5\right.$, $\left.J_{4,5} 7.8\right), 4.34\left(1 \mathrm{H}, \mathrm{dd}, \mathrm{H} 3, J_{3,4} 1.5, J_{3,2} 6.9\right), 4.55\left(1 \mathrm{H}, \mathrm{ddd}, \mathrm{H} 2, J_{2,1}, 4.7, J_{2,3} 6.9, J_{2,1} 9.0\right) ;{ }^{13} \mathrm{C}$ NMR $\left(\mathrm{CD}_{3} \mathrm{CN}\right.$, $100 \mathrm{MHz}$ ): 4.7 (C1), $25.7\left(\mathrm{CH}_{3}\right), 27.2\left(\mathrm{CH}_{3}\right), 27.4\left(\mathrm{CH}_{3}\right), 27.6\left(\mathrm{CH}_{3}\right), 64.6(\mathrm{C} 7), 74.4(\mathrm{C} 6), 77.0$ (C3), 77.8 (C5), 78.9 (C4) 79.2 (C2), $109.2\left(\underline{\mathrm{C}}\left(\mathrm{CH}_{3}\right)_{2}\right), 110.3\left(\underline{\mathrm{C}}\left(\mathrm{CH}_{3}\right)_{2}\right) ; \mathrm{m} / \mathrm{z}$ (ESI+ve): $425\left([\mathrm{M}+\mathrm{Na}]^{+}, 100 \%\right) ; \mathrm{HRMS}$ (ESI+ve): found $425.0431[\mathrm{M}+\mathrm{Na}]^{+} ; \mathrm{C}_{13} \mathrm{H}_{23} \mathrm{NaO}_{6} \mathrm{I}^{+}$requires 425.0432 .

\section{1-Deoxy-2,3:4,5-di-O-isopropylidene-D-glycero-D-gulo-heptitol 29}<smiles>CC1OC(C)(C)OC1C1OC(C)(C)OC1C(O)CO</smiles>

Palladium on charcoal (10\% wt., $500 \mathrm{mg}$ ) and triethylamine $(2.0 \mathrm{~mL}, 14.4 \mathrm{mmol})$ were added to a solution of 28 ( $5.8 \mathrm{~g}, 14.4 \mathrm{mmol})$ in methanol $(40 \mathrm{~mL})$. The reaction mixture was flushed with nitrogen, argon and hydrogen gas sequentially. Then the reaction mixture was stirred vigorously at room temperature under hydrogen atmosphere for $12 \mathrm{~h}$ until TLC (cyclohexane/ethyl acetate 1:1) showed the formation of one product $\left(R_{f} 0.25\right)$ and the disappearance of starting material $\left(R_{f} 0.36\right)$. After the reaction mixture had been filtered and evaporated to dryness in vacuo, the residue was dissolved in ethyl acetate $(50 \mathrm{~mL}$ ) and washed with water (aq., sat, $50 \mathrm{~mL}$ ). Then the aqueous layer was back extracted with ethyl acetate $(2 \times 50 \mathrm{~mL})$. The organic phase was dried $\left(\mathrm{MgSO}_{4}\right)$, filtered and the solvent was removed in vacuo to yield desired product 29 (3.2 g, 83\%) as a crystalline solid without further purification.

m.p. $40-42{ }^{\circ} \mathrm{C} ;[\alpha]_{\mathrm{D}}{ }^{20}=+59(c, 1.0$ in $\mathrm{MeOH}) ; v_{\max }\left(\right.$ thin film): $3420(\mathrm{br}, \mathrm{OH}) ;{ }^{1} \mathrm{H}$ NMR $\left(\mathrm{CD}_{3} \mathrm{OD}, 400 \mathrm{MHz}\right)$ : $1.36\left(3 \mathrm{H}, \mathrm{s}, \mathrm{CH}_{3}\right), 1.38\left(3 \mathrm{H}, \mathrm{s}, \mathrm{CH}_{3}\right), 1.41(3 \mathrm{H}, \mathrm{d}, \mathrm{H} 1, \mathrm{~J} 6.0), 1.42\left(3 \mathrm{H}, \mathrm{s}, \mathrm{CH}_{3}\right), 1.50\left(3 \mathrm{H}, \mathrm{s}, \mathrm{CH}_{3}\right), 3.58(1 \mathrm{H}$, 
dd, H7, J7,6 6.4, Jgem 11.0), 3.63 (1H, ddd, H6, J6,7 2.6, J6,7 6.4, J6,5 7.8), 3.77 (1H, dd, H7', J7,6 2.6, Jgem 11.0), $3.92\left(1 \mathrm{H}, \mathrm{t}, \mathrm{H} 5, J_{5,4}=J_{5,6} 7.8\right), 4.00\left(1 \mathrm{H}, \mathrm{dd}, \mathrm{H} 4, J_{4,3} 0.7, J_{4,5} 7.8\right), 4.29\left(1 \mathrm{H}, \mathrm{br}-\mathrm{d}, \mathrm{H} 3, J_{3,2} 6.6\right), 4.46$ (1H, quint, $\left.\mathrm{H} 2, J_{2,3}=J_{2,1} 6.6\right) ;{ }^{13} \mathrm{C} \mathrm{NMR}\left(\mathrm{CD}_{3} \mathrm{OD}, 100 \mathrm{MHz}\right): 15.7(\mathrm{C} 1), 25.8\left(\mathrm{CH}_{3}\right), 27.2\left(\mathrm{CH}_{3}\right), 27.6\left(\mathrm{CH}_{3}\right), 27.7\left(\mathrm{CH}_{3}\right)$, 65.4 (C7), 74.5 (C2), 75.5 (C6), 78.0 (C5), 78.1 (C3) 80.2 (C4), $109.3\left(\underline{\mathrm{C}}\left(\mathrm{CH}_{3}\right)_{2}\right), 110.7\left(\underline{\mathrm{C}}\left(\mathrm{CH}_{3}\right)_{2}\right) ; m / z$ (ESI+ve): $299\left([\mathrm{M}+\mathrm{Na}]^{+}, 100 \%\right)$; HRMS (ESI+ve): Found $299.1466[\mathrm{M}+\mathrm{Na}]^{+} ; \mathrm{C}_{13} \mathrm{H}_{24} \mathrm{NaO}_{6}{ }^{+}$requires 299.1465.

\section{6-Deoxy-2,3:4,5-di-O-isopropylidene-L-glucose 30}<smiles>CC1OC(C)(C)OC1C1OC(C)(C)OC1C=O</smiles>

Silica gel-supported $\mathrm{NaIO}_{4}$ (23.2 g) was added portionwise to a vigorously stirred solution of 29 (3.2 g, $11.6 \mathrm{mmol}$ ) in DCM (50 mL). After 2 hours, TLC analysis (ethyl acetate) showed no remaining starting material $\left(\mathrm{R}_{\mathrm{f}} 0.53\right)$ and formation of a single product $\left(\mathrm{R}_{\mathrm{f}} 0.66\right)$. The mixture was dried $\left(\mathrm{MgSO}_{4}\right)$, filtered and the silica gel was thoroughly washed with DCM (4 x $50 \mathrm{~mL})$. The solvents were removed in vacuo to afford the aldehyde $\mathbf{3 0}$ (2.6 $\mathrm{g}, 93 \%)$ as a light brown foam.

$[\alpha]_{\mathrm{D}^{20}}=+42\left(c, 1.3\right.$ in $\left.\mathrm{CHCl}_{3}\right) ; v_{\max }$ (thin film): 3423 (br, OH), $1730(\mathrm{~s}, \mathrm{C}=0) ;{ }^{1} \mathrm{H} \mathrm{NMR}\left(\mathrm{CDCl}_{3}, 400 \mathrm{MHz}\right):$ $1.37\left(3 \mathrm{H}, \mathrm{s}, \mathrm{CH}_{3}\right), 1.38\left(3 \mathrm{H}, \mathrm{d}, \mathrm{H} 6, J_{6,5} 6.6\right), 1.42\left(3 \mathrm{H}, \mathrm{s}, \mathrm{CH}_{3}\right), 1.51\left(3 \mathrm{H}, \mathrm{s}, \mathrm{CH}_{3}\right), 1.52\left(3 \mathrm{H}, \mathrm{s}, \mathrm{CH}_{3}\right), 3.97(1 \mathrm{H}$, dd, H3, J3,4 2.0, $J_{3,2} 7.9$ ), $4.10\left(1 \mathrm{H}, \mathrm{dd}, \mathrm{H} 4, J_{4,3} 2.0, J_{4,5} 6.6\right.$ ), 4.34 (1H, dd, H2, $J_{2,1} 1.8, J_{2,3} 7.9$ ), 4.41 (1H, quint, $\left.\mathrm{H} 5, J_{5,4}=J_{5,6} 6.6\right), 9.82\left(1 \mathrm{H}, \mathrm{d}, \mathrm{H} 1, J_{1,2} 1.8\right) ;{ }^{13} \mathrm{C} \mathrm{NMR}\left(\mathrm{CDCl}_{3}, 100 \mathrm{MHz}\right): 15.0(\mathrm{C} 6), 25.4\left(\mathrm{CH}_{3}\right), 26.3\left(\mathrm{CH}_{3}\right)$, $26.7\left(\mathrm{CH}_{3}\right), 26.8\left(\mathrm{CH}_{3}\right), 72.6(\mathrm{C} 5), 75.7,75.8(\mathrm{C} 2, \mathrm{C} 3), 81.3(\mathrm{C} 4), 108.5\left(\underline{\mathrm{C}}\left(\mathrm{CH}_{3}\right)_{2}\right), 111.7\left(\underline{\mathrm{C}}\left(\mathrm{CH}_{3}\right)_{2}\right), 201.7$ (C1); $m / z$ (ESI+ve): $299\left(\left[\mathrm{M}+\mathrm{MeOH}+\mathrm{Na}^{+}, 100 \%\right)\right.$, HRMS (ESI+ve): found $267.1209\left([\mathrm{M}+\mathrm{Na}]^{+}\right)$; $\mathrm{C}_{12} \mathrm{H}_{20} \mathrm{NaO}_{5}{ }^{+}$requires 267.1203.

\section{6-Deoxy-L-glucose [L-quinovose] 31}<smiles>CC(O)[C@@H](C)[C@@H](O)C=O</smiles>

$\mathrm{OH} \mathrm{OH}$

DOWEX ${ }^{\circledR} 50 \mathrm{WX} 8-200(\sim 300 \mathrm{mg}$, pre-washed with water) was added into a solution of 30 (2.3 g, 9.4 $\mathrm{mmol}$ ) in water/1,4-dioxane (10 mL, 1:1). After stirring at room temperature for $18 \mathrm{~h}$, TLC showed the disappearance of starting material $\left(\mathrm{R}_{\mathrm{f}} 0.66\right)$. Then resin was filtered off and the solvent was removed in vacuo to obtain deprotected sugar 31 (1.5 g, 100\%) as a light yellow syrup that was recrystallized from acetonitrile/ethanol (6:1) to obtain $31(1.0 \mathrm{~g}, 67 \%)$ as a crystalline solid $(\alpha / \beta$ pyranoside ratio $2: 5)$. $1 \mathrm{H}$ NMR of 6-deoxy-L-glucose $\mathbf{3 1}$ is identical with that of commercial sample of 6-deoxy-D-glucose.

m.p. $126-128{ }^{\circ} \mathrm{C} ;[\alpha]_{\mathrm{D}} 20=-47.7\left(c, 1.12\right.$ in water), [lit. ${ }^{9}[\alpha]_{\mathrm{D}^{20}}=-40$ (c 1.39 in water)]; $v_{\max }$ (thin film):

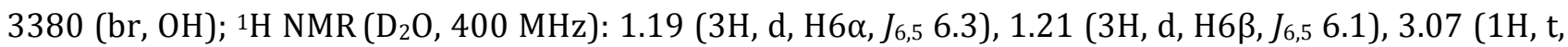

9 Liu, Z.; Yoshihara, A.; Kelly, C.; Heap, T. J.; Marqvorsen, M. H. S.; Jenkinson, S. F.; Wormald, M. R.; Otero, J. M.; Estévez, A.; Kato, A.; Fleet, G. W. J.; Estévez, R.; Izumori, K. Chem. Eur. J., 2016, accepted. 
$\left.\mathrm{H} 4 \alpha, J_{4,3}=J_{4,5} 9.5\right), 3.08\left(1 \mathrm{H}, \mathrm{t}, \mathrm{H} 4 \beta, J_{4,3}=J_{4,5} 9.3\right), 3.17\left(1 \mathrm{H}, \mathrm{dd}, \mathrm{H} 2 \beta, J_{2,1} 7.9, J_{2,3} 9.3\right), 3.36\left(1 \mathrm{H}, \mathrm{t}, \mathrm{H} 3 \beta, J_{3,2}=\right.$ $J_{3,4} 9.3$ ), $3.42\left(1 \mathrm{H}, \mathrm{dq}, \mathrm{H} 5 \beta, J_{5,6} 6.2, J_{5,4} 9.5\right), 3.47\left(1 \mathrm{H}, \mathrm{dd}, \mathrm{H} 2 \alpha, J_{2,1} 3.8, J_{2,3} 9.9\right.$ ), $3.58\left(1 \mathrm{H}, \mathrm{t}, \mathrm{H} 3 \alpha, J_{3,2}=J_{3,4}\right.$ 9.5), 3.85 (1H, dq, $\left.H 5 \alpha, J_{5,6} 6.3, J_{5,4} 9.6\right), 4.55\left(1 \mathrm{H}, \mathrm{d}, \mathrm{H} 1 \beta, J_{1,2} 7.9\right.$ ), $5.10\left(1 \mathrm{H}, \mathrm{d}, \mathrm{H} 1 \alpha, J_{1,2} 3.8\right) ;{ }^{13} \mathrm{C}$ NMR $\left(\mathrm{D}_{2} \mathrm{O}\right.$,

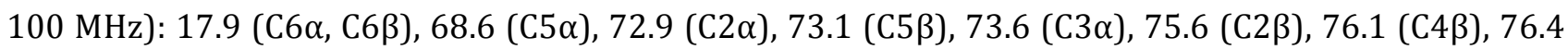

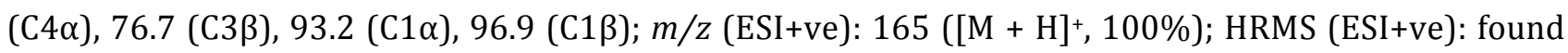
165.0757 [M+ H]+; $\mathrm{C}_{6} \mathrm{H}_{13} \mathrm{O}_{5}+$ requires 165.0757.

\section{6-Deoxy-2,3:4,5-di-O-isopropylidene-L-glucitol 32}<smiles>CC1OC(C)(C)OC1C1OC(C)(C)OC1C</smiles>

Sodium borohydride (19 $\mathrm{mg}, 0.49 \mathrm{mmol}$ ) was added to a solution of $\mathbf{3 0}$ (100 $\mathrm{mg}, 0.41 \mathrm{mmol}$ ) in methanol $(4 \mathrm{~mL})$. The reaction mixture was stirred at room temperature for 2 hours until mass spectrometry showed the completion of reaction $([\mathrm{M}+\mathrm{Na}]+279)$. After neutralizing the reaction mixture with acetic acid $(\sim 0.5 \mathrm{~mL})$, the solvent was removed in vacuo to yield a residue that was dissolved in ethyl acetate $(10 \mathrm{~mL})$. The resulting solution was washed with water $(3 \mathrm{x} 10 \mathrm{~mL})$ and the organic phase was dried $\left(\mathrm{MgSO}_{4}\right)$, filtered and solvent was removed in vacuo to yield protected glucitol 32 (86 $\mathrm{mg}, 85 \%)$ as a colorless oil.

$[\alpha]_{\mathrm{D}^{20}}=+57\left(c, 1.2\right.$ in $\left.\mathrm{CHCl}_{3}\right) ; v_{\max }$ (thin film): 3461 (br, OH); ${ }^{1} \mathrm{H} \mathrm{NMR}\left(\mathrm{CDCl}_{3}, 400 \mathrm{MHz}\right): 1.37\left(3 \mathrm{H}, \mathrm{s}, \mathrm{CH}_{3}\right)$, $1.40\left(3 \mathrm{H}, \mathrm{d}, \mathrm{H} 6, J_{6,5} 6.6\right), 1.42\left(3 \mathrm{H}, \mathrm{s}, \mathrm{CH}_{3}\right), 1.46\left(3 \mathrm{H}, \mathrm{s}, \mathrm{CH}_{3}\right), 1.53\left(3 \mathrm{H}, \mathrm{s}, \mathrm{CH}_{3}\right), 3.62\left(1 \mathrm{H}, \mathrm{dd}, \mathrm{H} 1, J_{1,2}\right.$ 3.4,

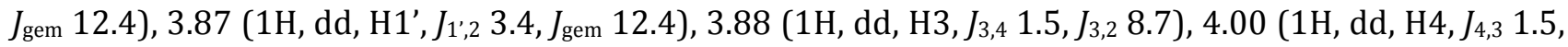
$J_{4,5}$ 6.7), $4.08\left(1 \mathrm{H}, \mathrm{dt}, \mathrm{H} 2, J_{2,1}=J_{2,1} \cdot 3.4, J_{2,3} 8.8\right), 4.42\left(1 \mathrm{H}\right.$, quint, $\left.\mathrm{H} 5, J_{5,4}=J_{5,6} 6.6\right) ;{ }^{33} \mathrm{C} \mathrm{NMR}\left(\mathrm{CDCl}_{3}, 100\right.$ MHz): 15.1 (C6), $25.4\left(\mathrm{CH}_{3}\right), 26.7\left(\mathrm{CH}_{3}\right), 27.1\left(\mathrm{CH}_{3}\right), 27.2\left(\mathrm{CH}_{3}\right), 60.7$ (C1), $72.8(\mathrm{C} 5), 75.2,75.3(\mathrm{C} 3, \mathrm{C} 4)$, 77.5 (C2), $108.4\left(\underline{\mathrm{C}}\left(\mathrm{CH}_{3}\right)_{2}\right), 109.4\left(\underline{\mathrm{C}}\left(\mathrm{CH}_{3}\right)_{2}\right) ; \mathrm{m} / z(\mathrm{ESI}+\mathrm{ve}): 269\left([\mathrm{M}+\mathrm{Na}]^{+}, 100 \%\right) ; \mathrm{HRMS}(\mathrm{ESI}+\mathrm{ve})$ : found $269.1362[\mathrm{M}+\mathrm{Na}]^{+} ; \mathrm{C}_{12} \mathrm{H}_{22} \mathrm{NaO}_{5}+$ requires 269.1359 .

\section{6-Deoxy-L-glucitol [1-deoxy-D-gulitol] 33}<smiles>CC(O)C(O)C(O)C(O)CO</smiles>

DOWEX ${ }^{\circledR}$ 50WX8-200 ( $50 \mathrm{mg}$, pre-washed with water) was added into a solution of 32 (86 mg, 0.35 mmol) in water/1,4-dioxane (10 mL, 1:1). After stirring at room temperature for $20 \mathrm{~h}$, resin was filtered off and solvent was removed in vacuo to obtain 6-deoxy-L-glucitol 33 (58 mg, 100\%) as a colorless syrup.

$[\alpha]_{\mathrm{D}}{ }^{20}=+7.3\left(c, 0.98\right.$ in water) [lit.10 $[\alpha]_{\mathrm{D}^{27}}=+1.45$ (c 3.105 in ethanol)]; $v_{\max }$ (thin film): 3400 (br, OH); ${ }^{1} \mathrm{H}$ NMR (D $\left.20,400 \mathrm{MHz}\right): 1.22$ (3H, d, H6, J6,5 6.6), 3.51 (1H, dd, H4, J4,3 2.9, J4,5 6.9), 3.61 (1H, dd, H1, J1,2

10 Gillard, F.; Riehl, J. J. Tetrahedron Lett. 1983, 24, 587-8. 
6.4, Jgem 11.5), $3.72\left(1 \mathrm{H}, \mathrm{dd}, \mathrm{H} 1^{\prime}, J_{1^{\prime}, 2} 3.9, J_{\text {gem }} 11.5\right), 3.77$ - $3.81(2 \mathrm{H}, \mathrm{m}, \mathrm{H} 2, \mathrm{H} 3), 3.89\left(1 \mathrm{H}\right.$, quint, $\mathrm{H} 5, J_{5,4}=$ $J_{5,6} 6.6$ ); ${ }^{13} \mathrm{C} \mathrm{NMR}\left(\mathrm{CDCl}_{3}, 100 \mathrm{MHz}\right.$ ): 18.5 (C6), 63.1 (C1), 67.6 (C5), 70.5 (C2), 73.2 (C3), 75.5 (C4); m/z (ESI+ve): $189\left([\mathrm{M}+\mathrm{Na}]^{+}, 100 \%\right) ;$ HRMS (ESI+ve): found $189.0731[\mathrm{M}+\mathrm{Na}]^{+} ; \mathrm{C}_{6} \mathrm{H}_{14} \mathrm{NaO}_{5}{ }^{+}$requires 189.0733 . 


\section{NMR Spectra}

${ }^{1} \mathrm{H}$ NMR spectrum for $7\left(\mathrm{CDCl}_{3}, 400 \mathrm{MHz}\right)$

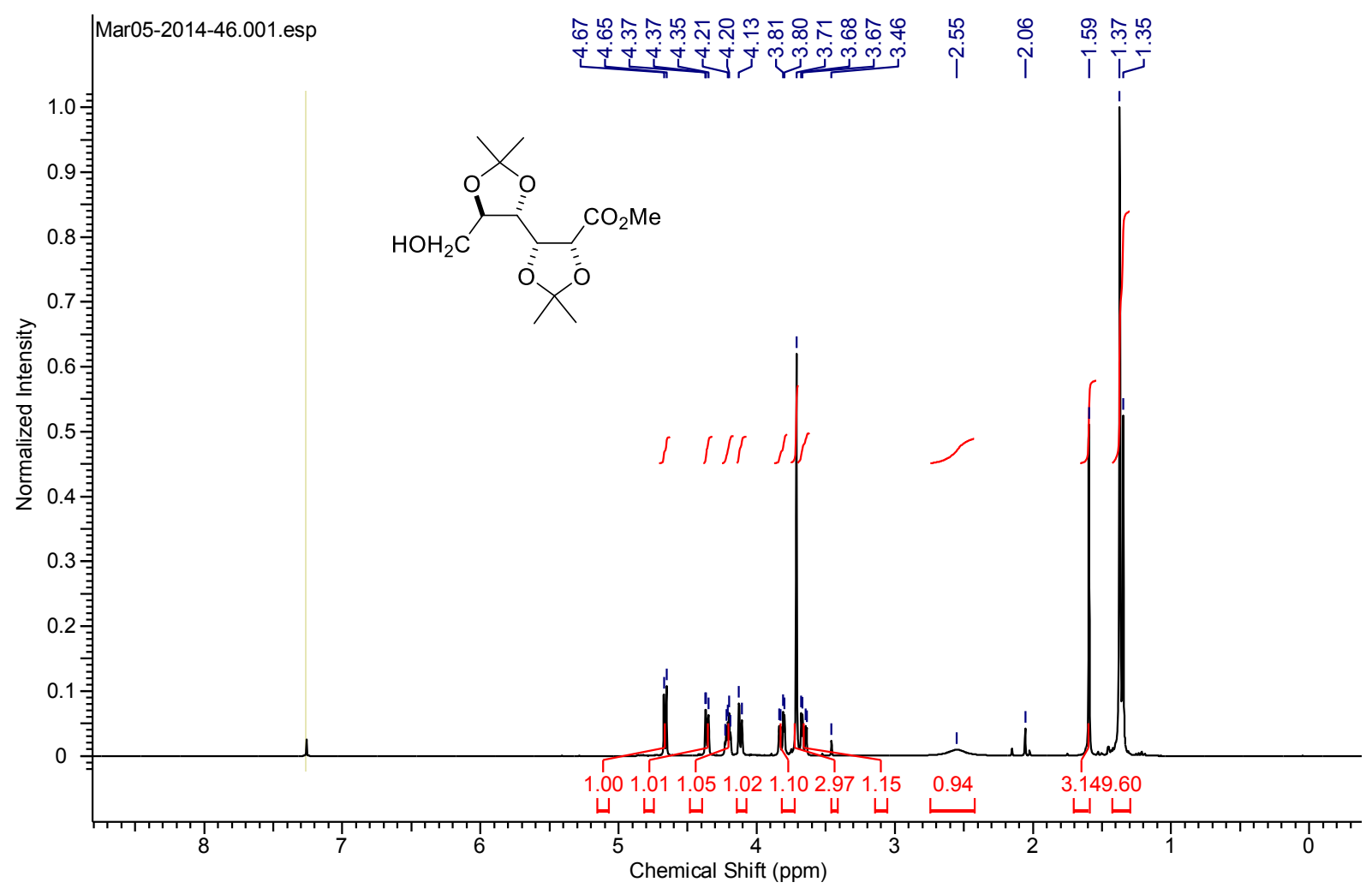


${ }^{13} \mathrm{C}$ NMR spectrum for $7\left(\mathrm{CDCl}_{3}, 100 \mathrm{MHz}\right)$

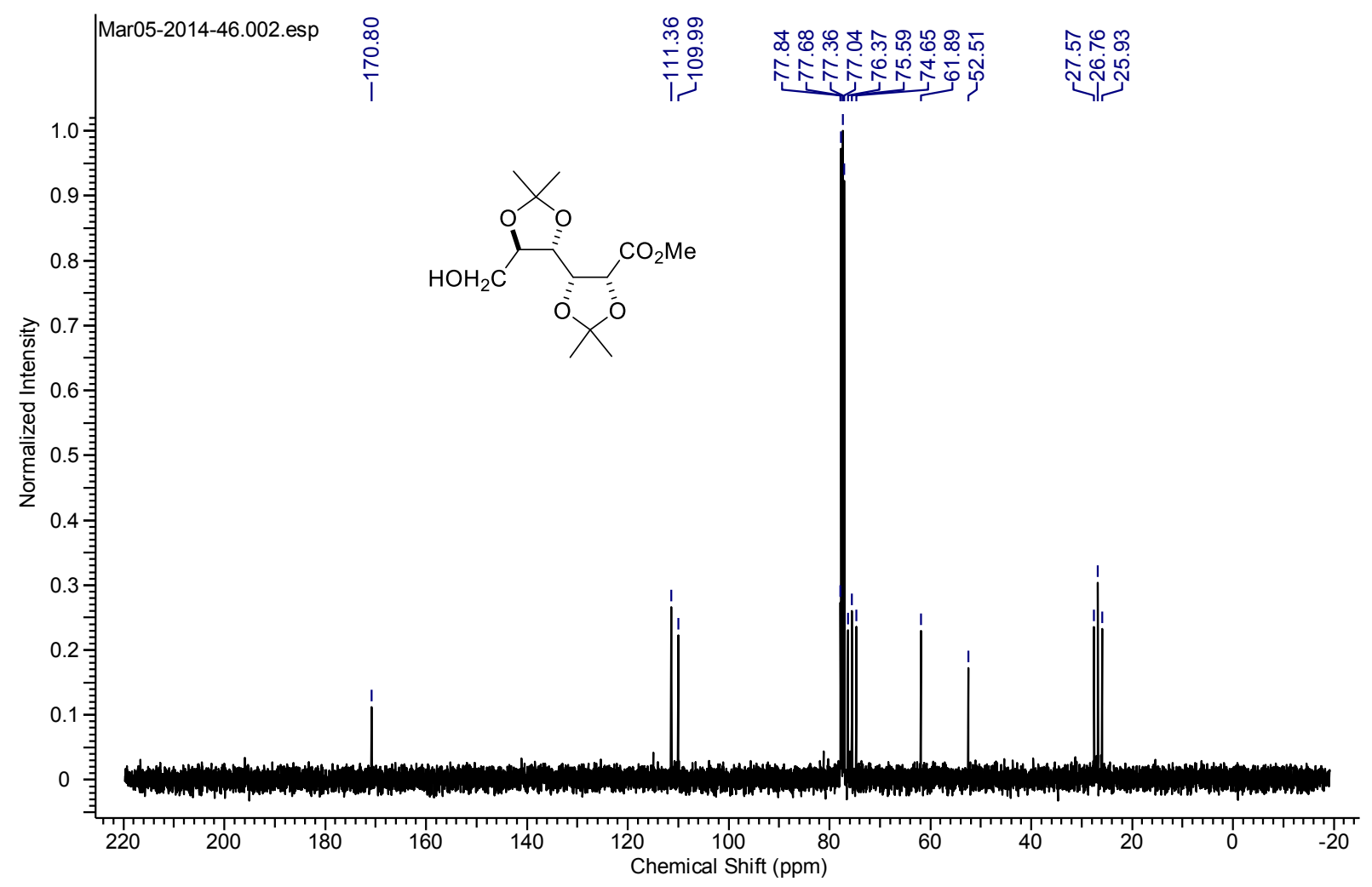


${ }^{1} \mathrm{H}$ NMR spectrum for $8\left(\mathrm{CDCl}_{3}, 400 \mathrm{MHz}\right)$

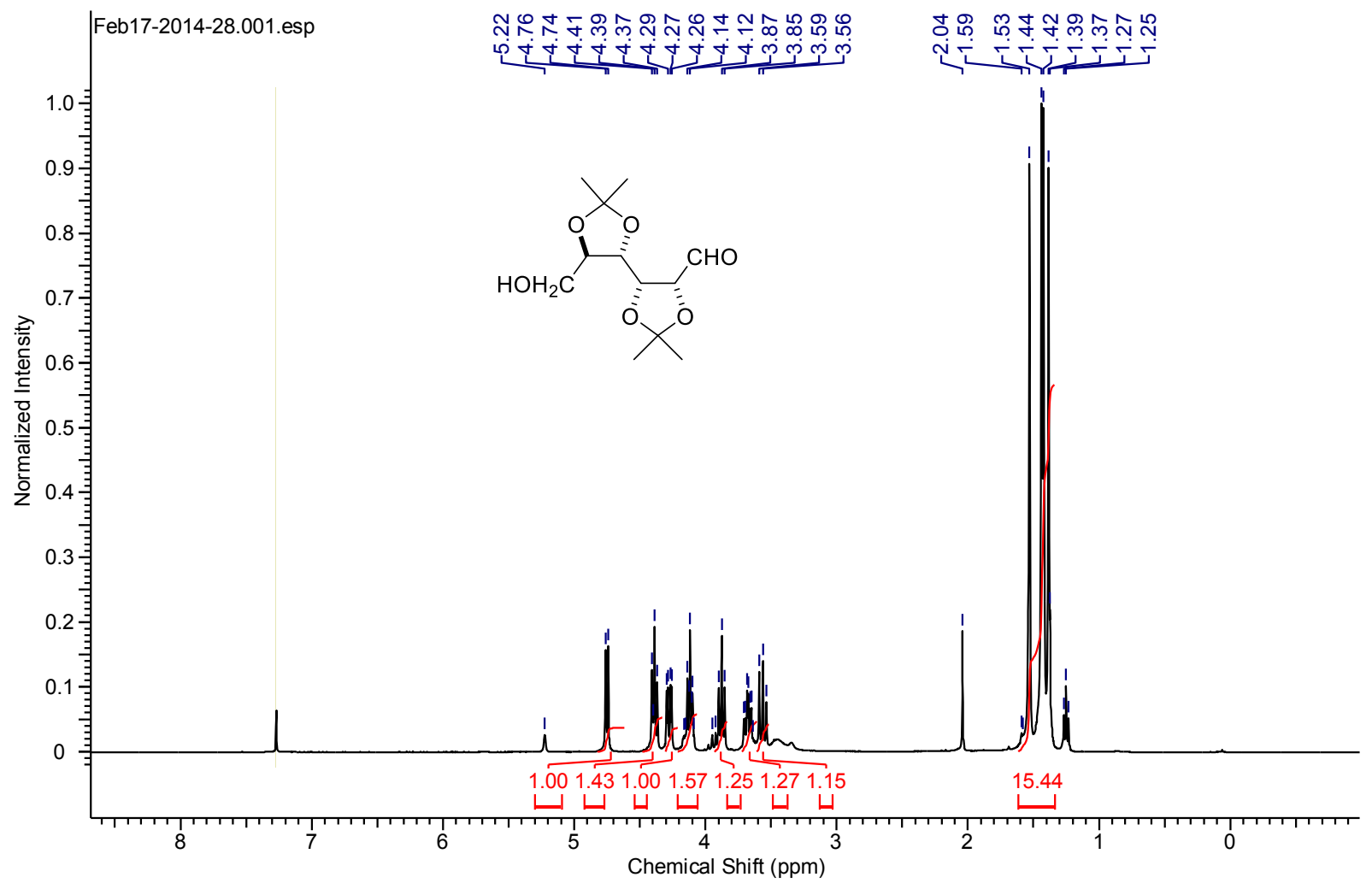


${ }^{13} \mathrm{C}$ NMR spectrum for $8\left(\mathrm{CDCl}_{3}, 100 \mathrm{MHz}\right)$

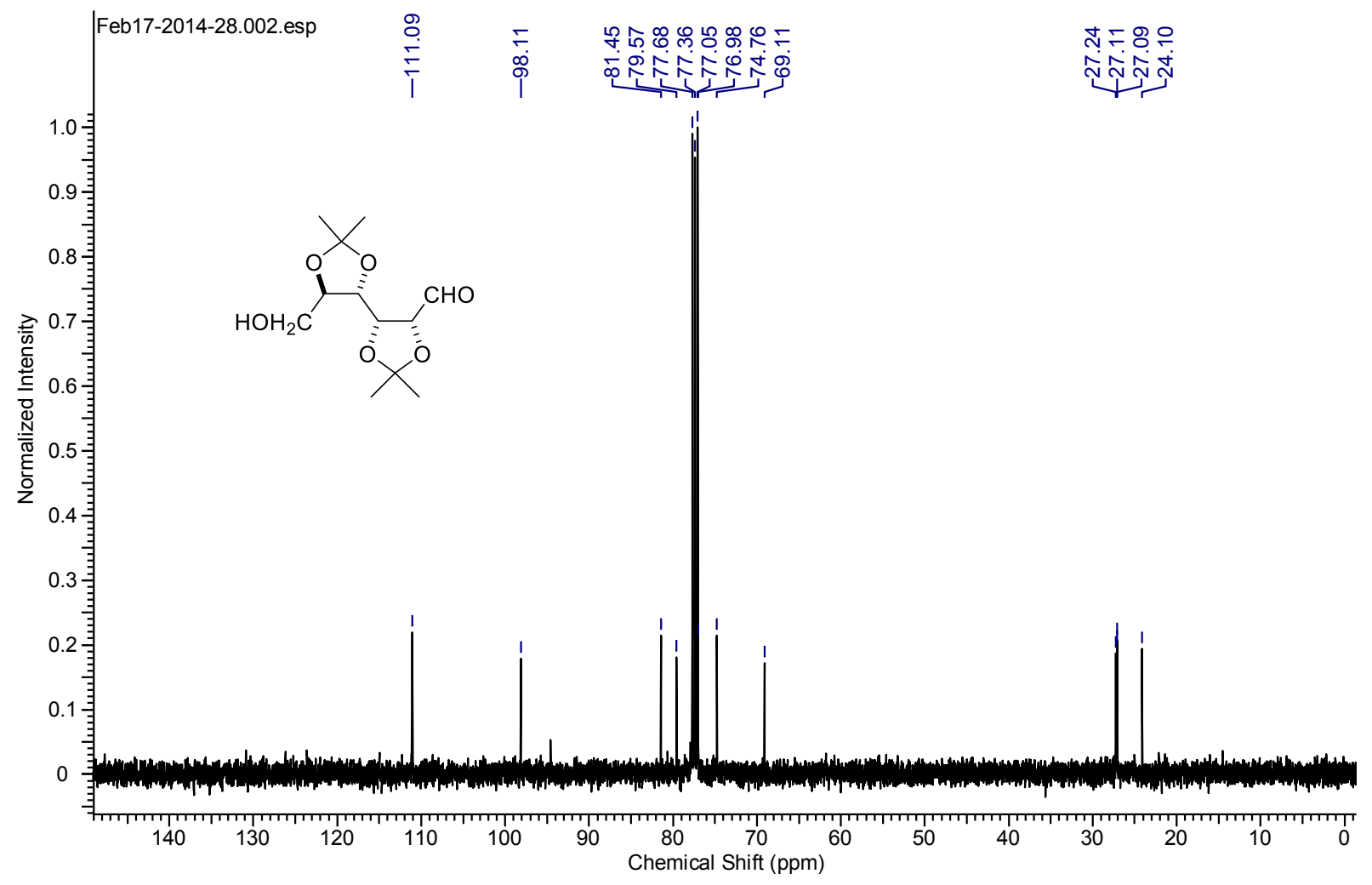


${ }^{1} \mathrm{H}$ NMR spectrum for $9\left(\mathrm{D}_{2} \mathrm{O}, 400 \mathrm{MHz}\right)$

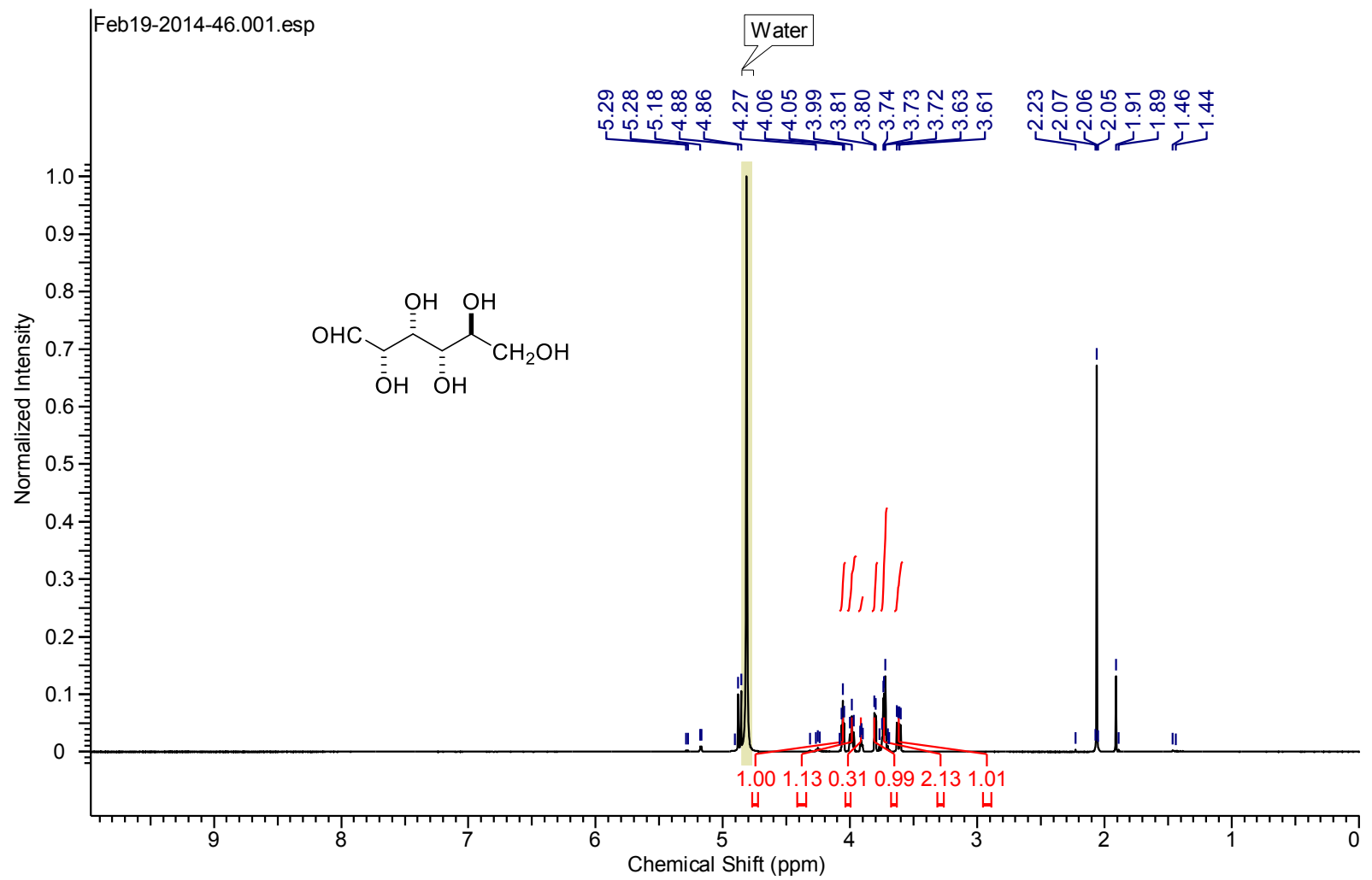


${ }^{13} \mathrm{C}$ NMR spectrum for $9\left(\mathrm{D}_{2} \mathrm{O}, 100 \mathrm{MHz}\right)$

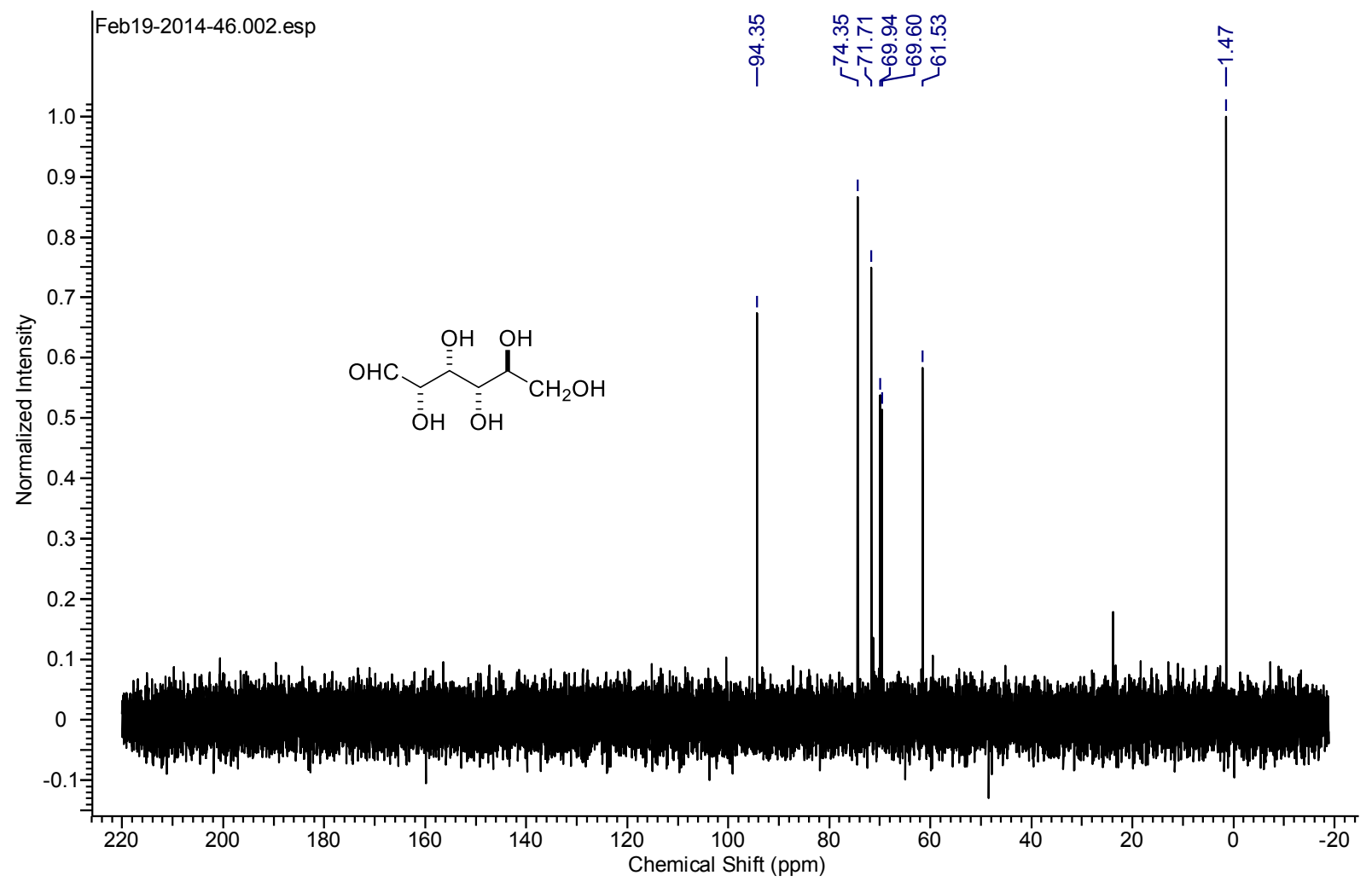


${ }^{1} \mathrm{H}$ NMR spectrum for commercial D-gulose $\left(\mathrm{D}_{2} \mathrm{O}, 400 \mathrm{MHz}\right)$

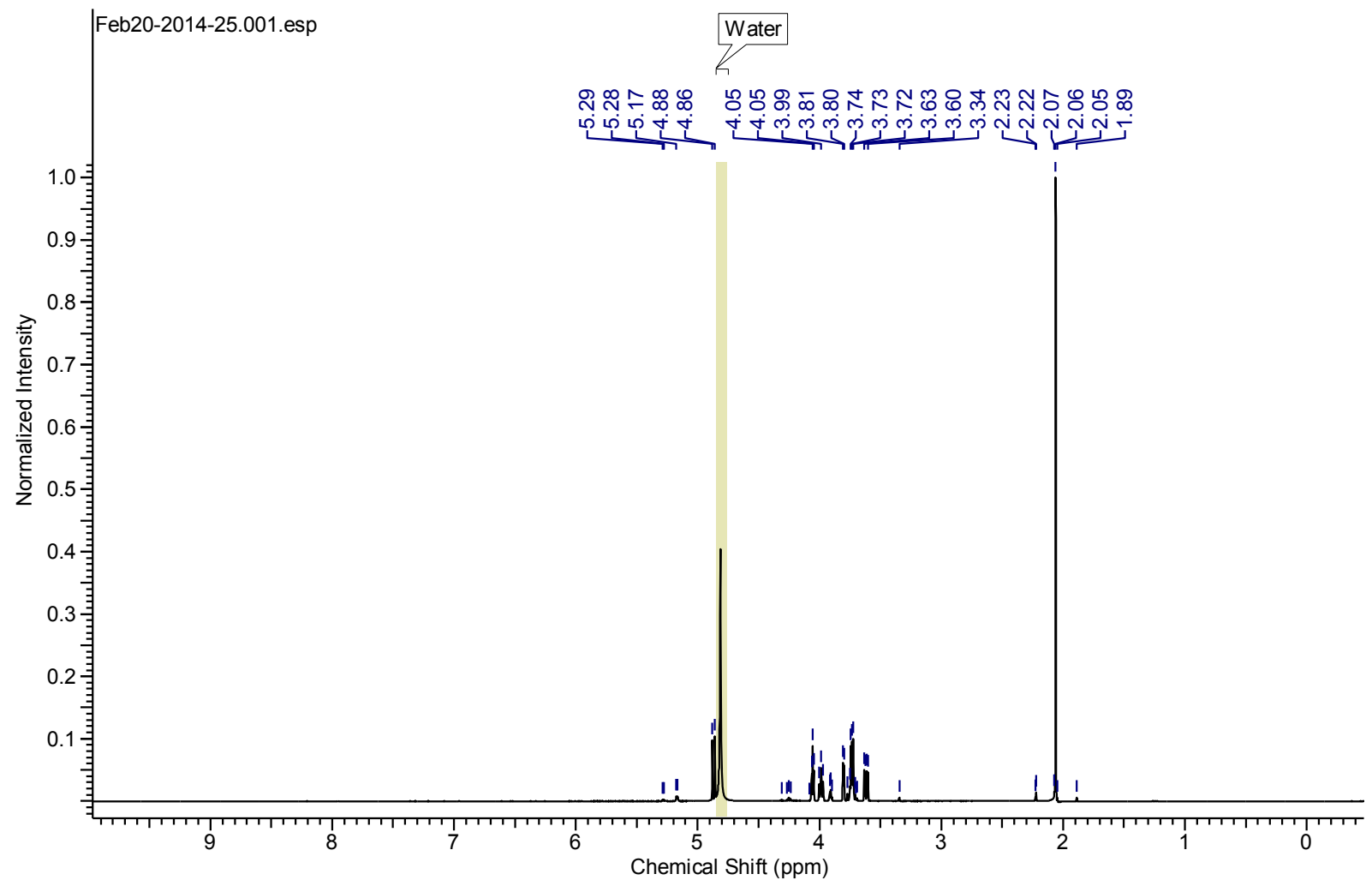


${ }^{13} \mathrm{C}$ NMR spectrum for commercial D-gulose $\left(\mathrm{D}_{2} \mathrm{O}, 100 \mathrm{MHz}\right)$

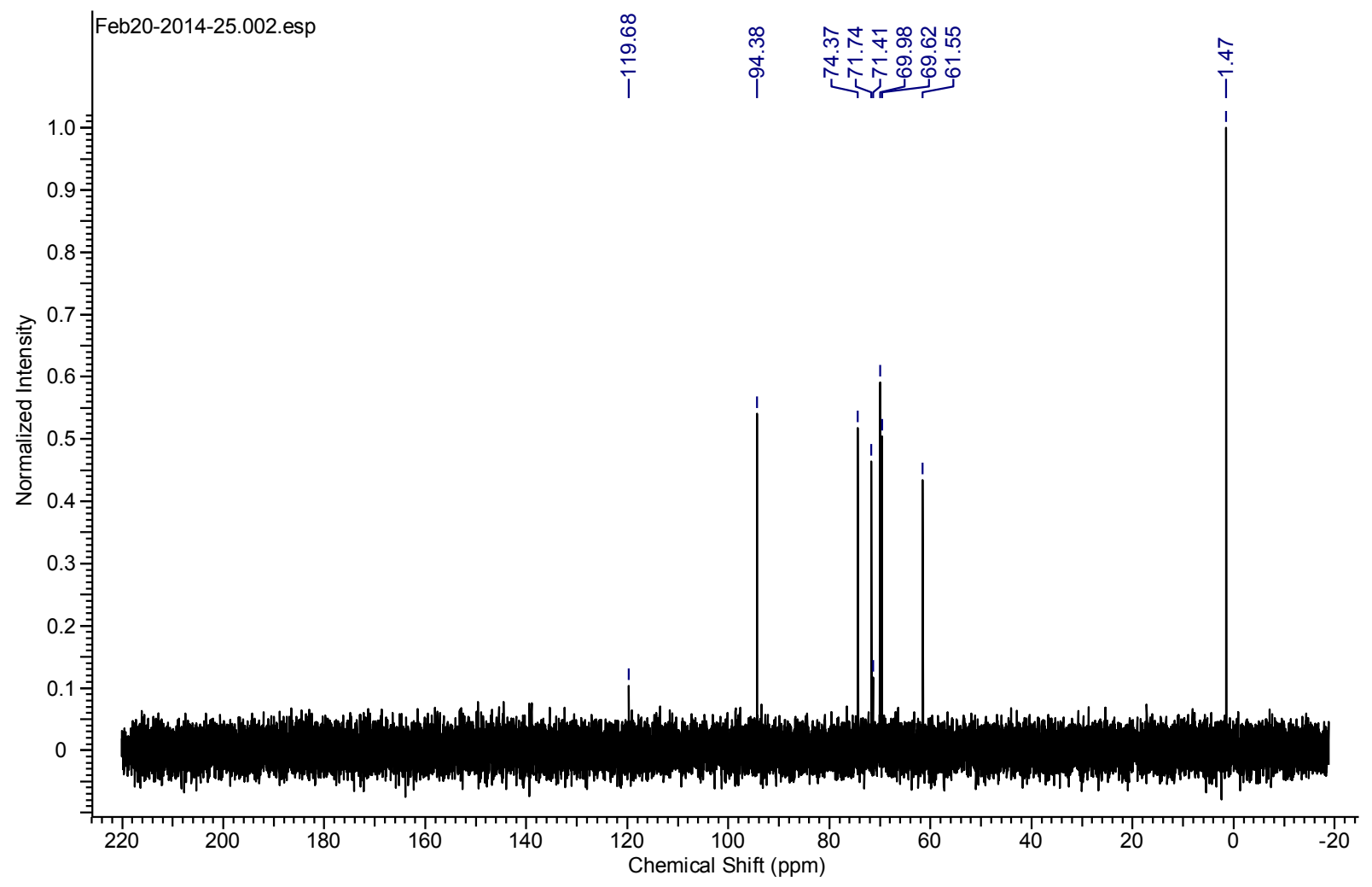


${ }^{1} \mathrm{H}$ NMR spectrum for $\mathbf{1 0}\left(\mathrm{CD}_{3} \mathrm{OD}, 400 \mathrm{MHz}\right)$

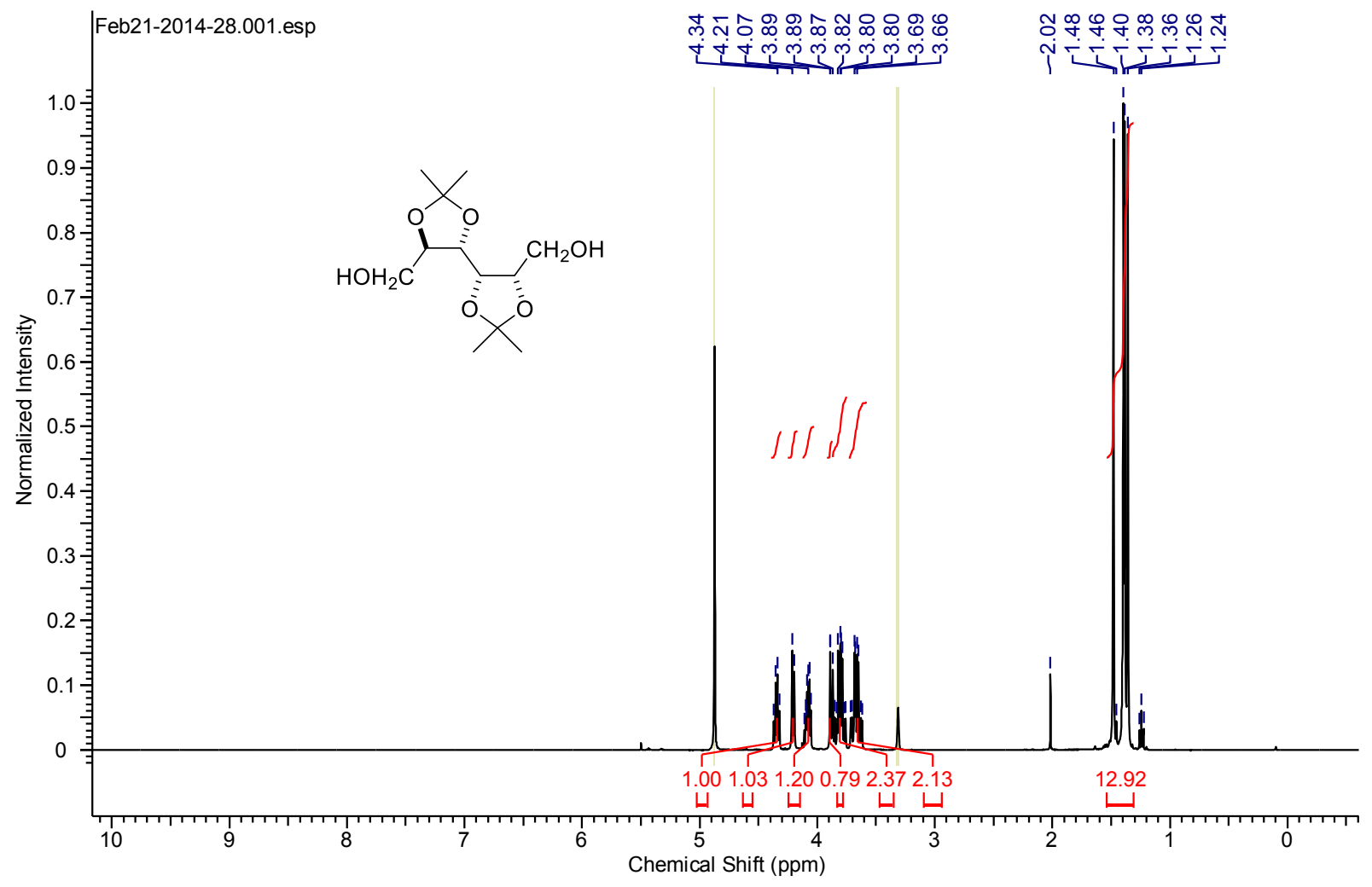


${ }^{13} \mathrm{C}$ NMR spectrum for $\mathbf{1 0}\left(\mathrm{CD}_{3} \mathrm{OD}, 100 \mathrm{MHz}\right)$

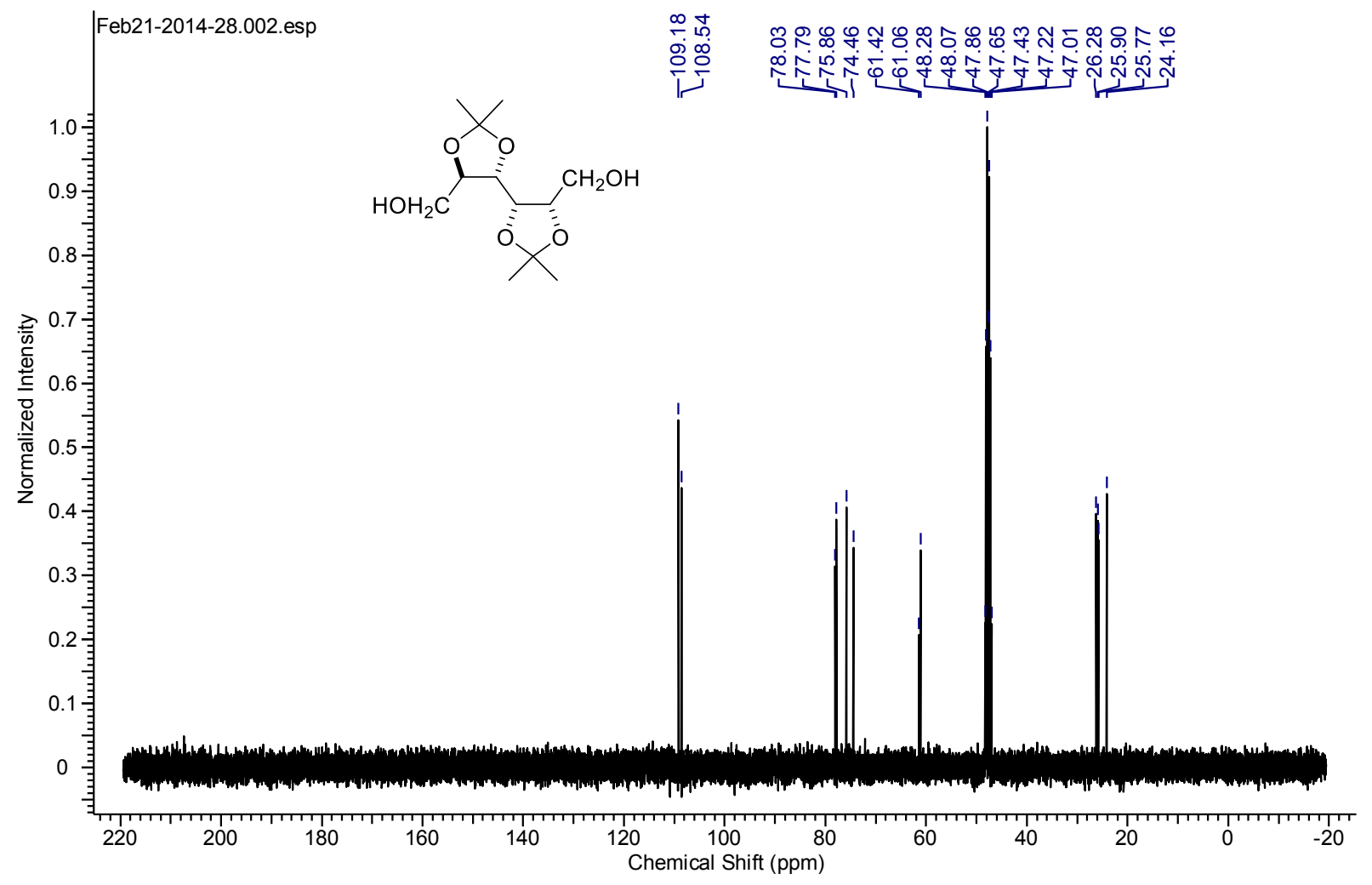


${ }^{1} \mathrm{H}$ NMR spectrum for $\mathbf{1 1}\left(\mathrm{D}_{2} \mathrm{O}, 400 \mathrm{MHz}\right)$

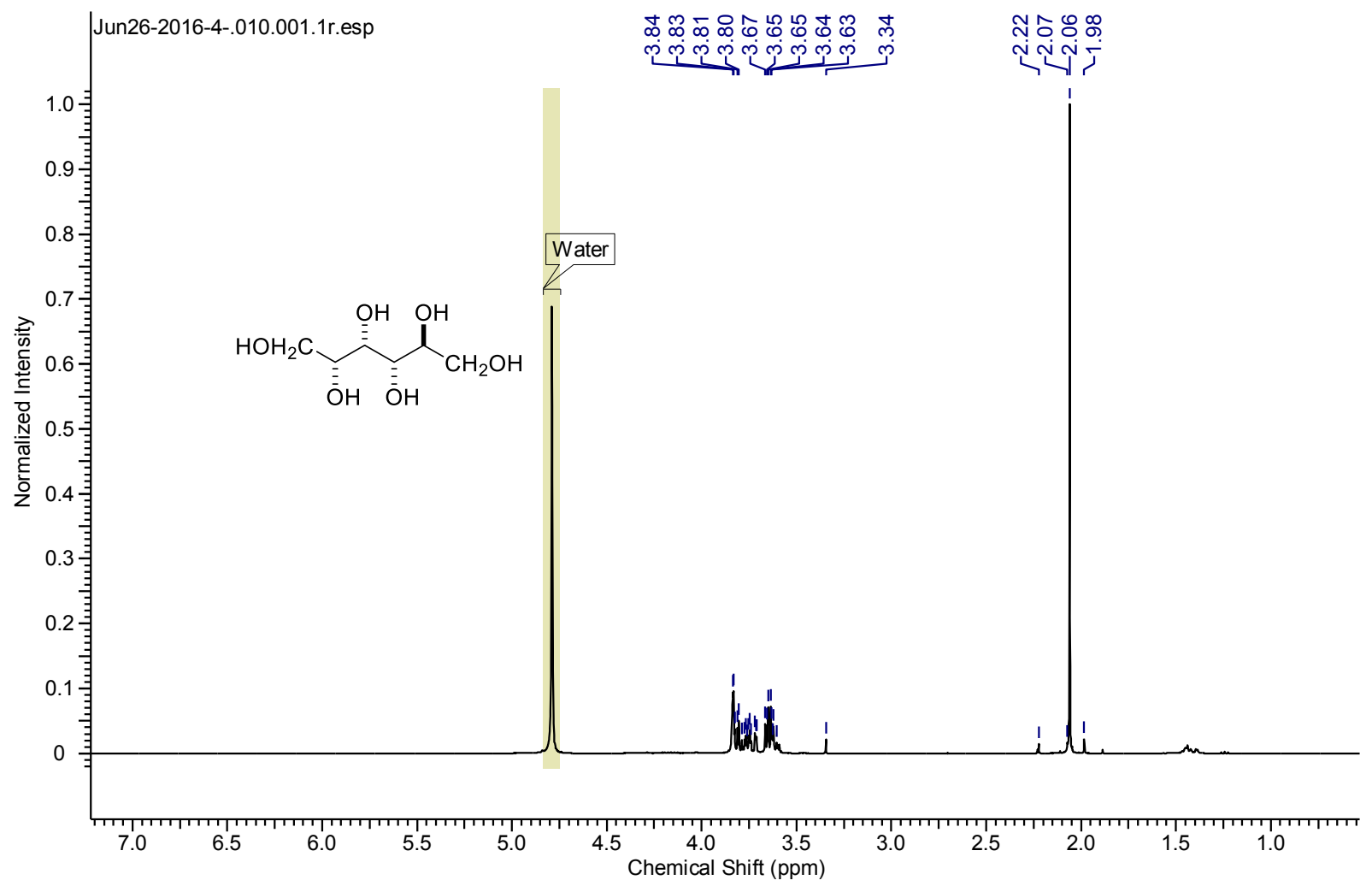


${ }^{13} \mathrm{C}$ NMR spectrum for $\mathbf{1 1}\left(\mathrm{D}_{2} \mathrm{O}, 100 \mathrm{MHz}\right)$

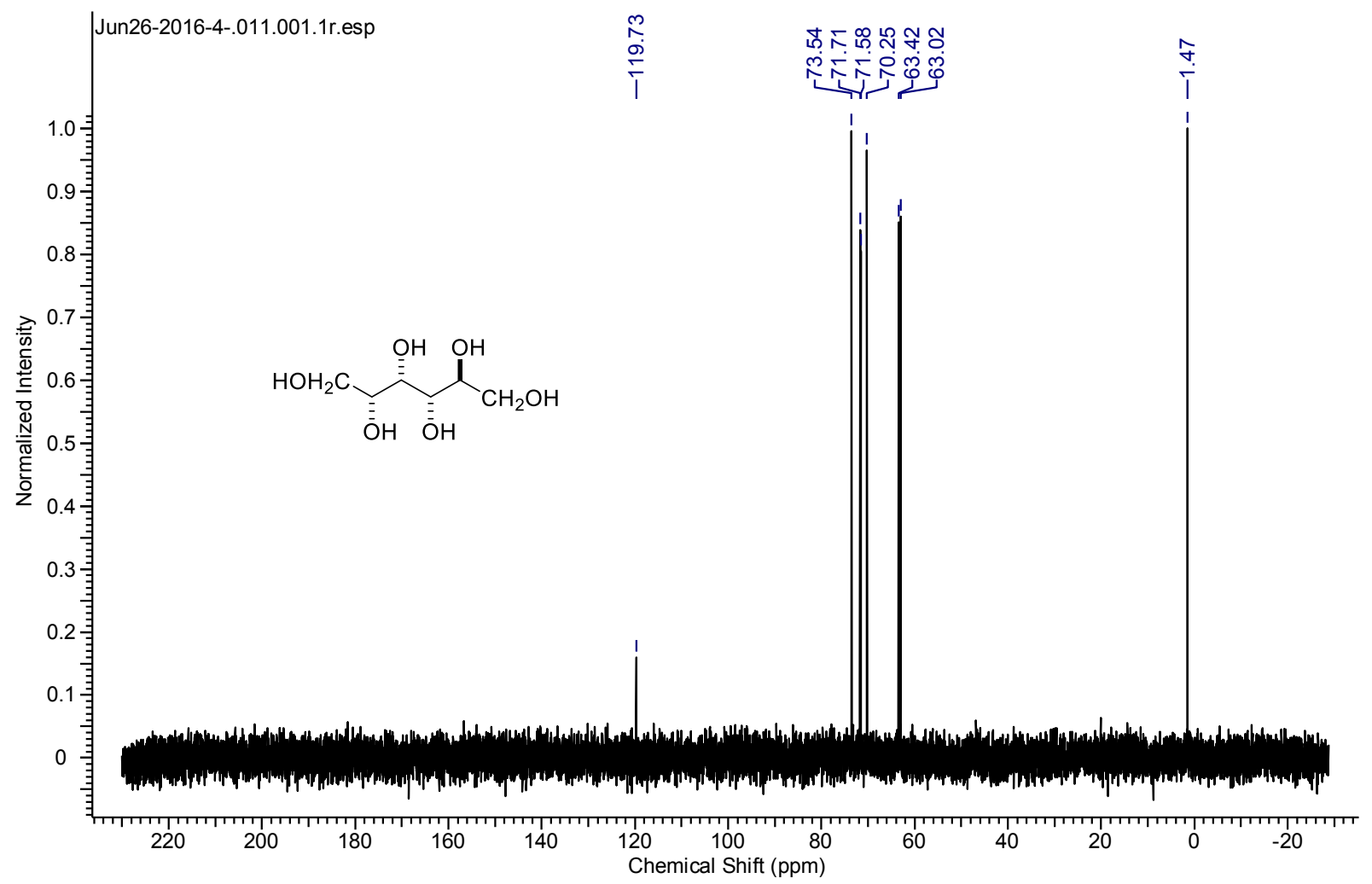


${ }^{1} \mathrm{H}$ NMR spectrum for commercial L-glucitol $\left(\mathrm{D}_{2} \mathrm{O}, 400 \mathrm{MHz}\right)$

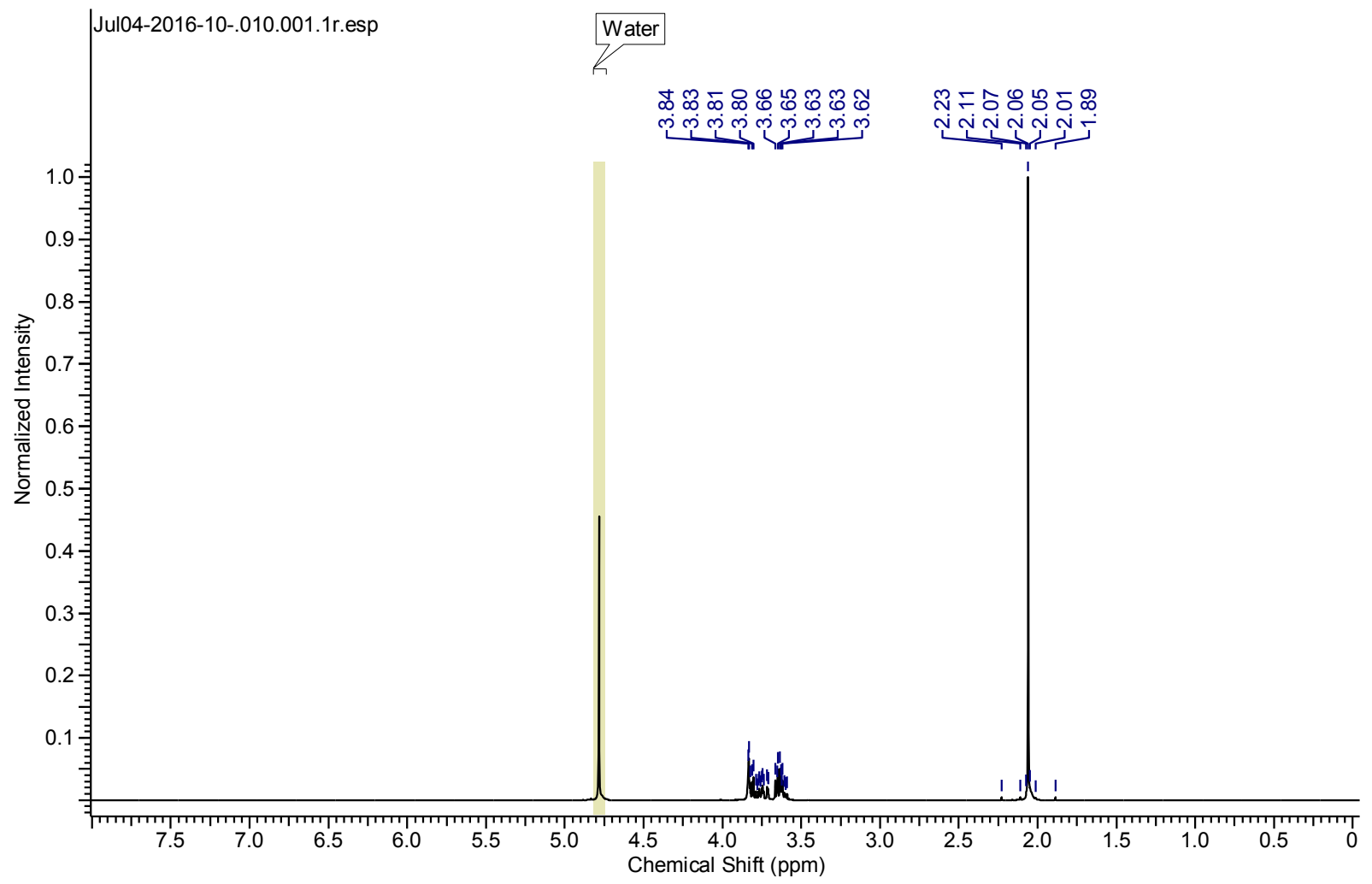


${ }^{13} \mathrm{C}$ NMR spectrum for commercial L-glucitol $\left(\mathrm{D}_{2} \mathrm{O}, 100 \mathrm{MHz}\right)$

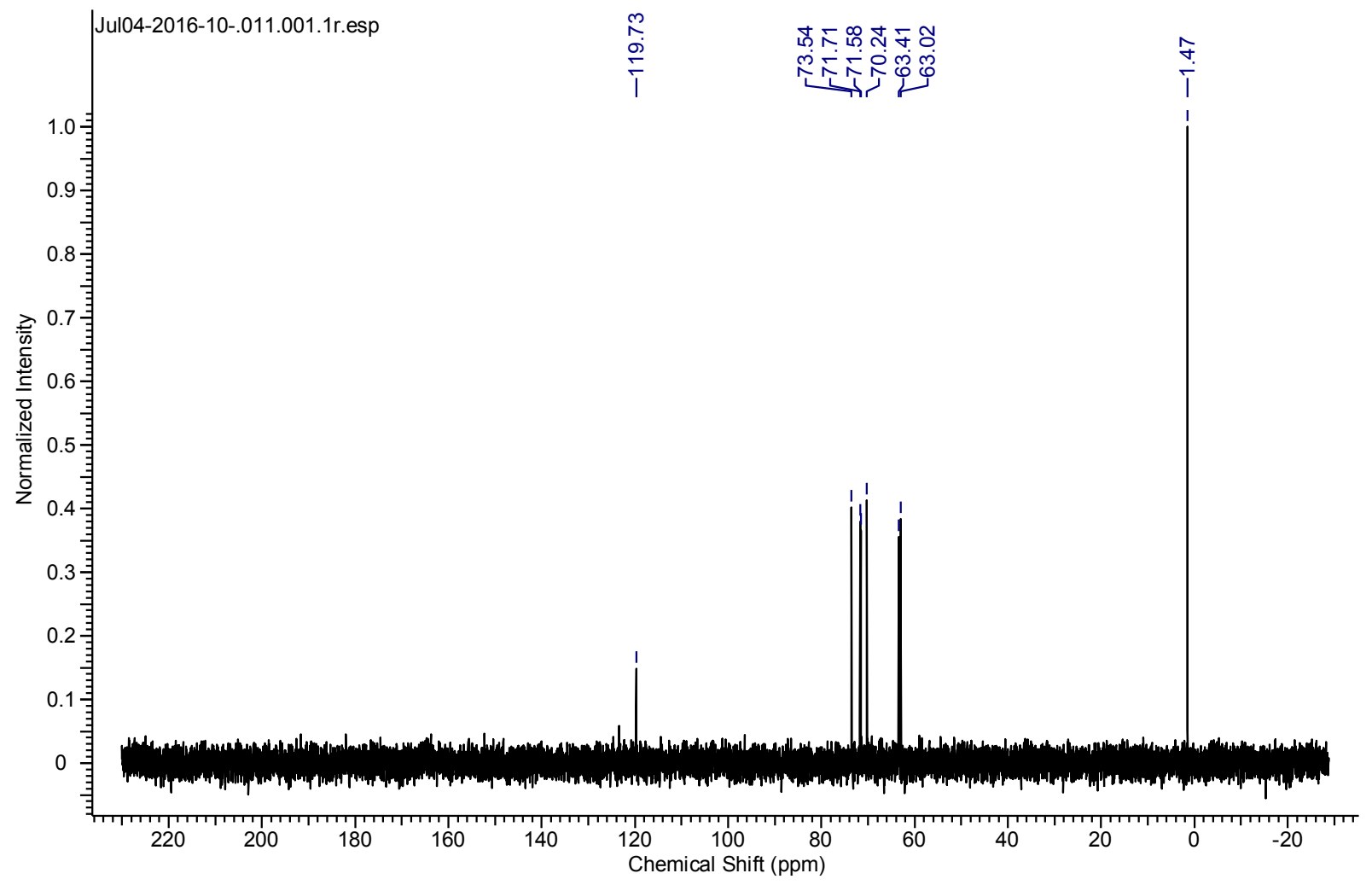


${ }^{1} \mathrm{H}$ NMR spectrum for $\mathbf{1 3}\left(\mathrm{CDCl}_{3}, 400 \mathrm{MHz}\right)$

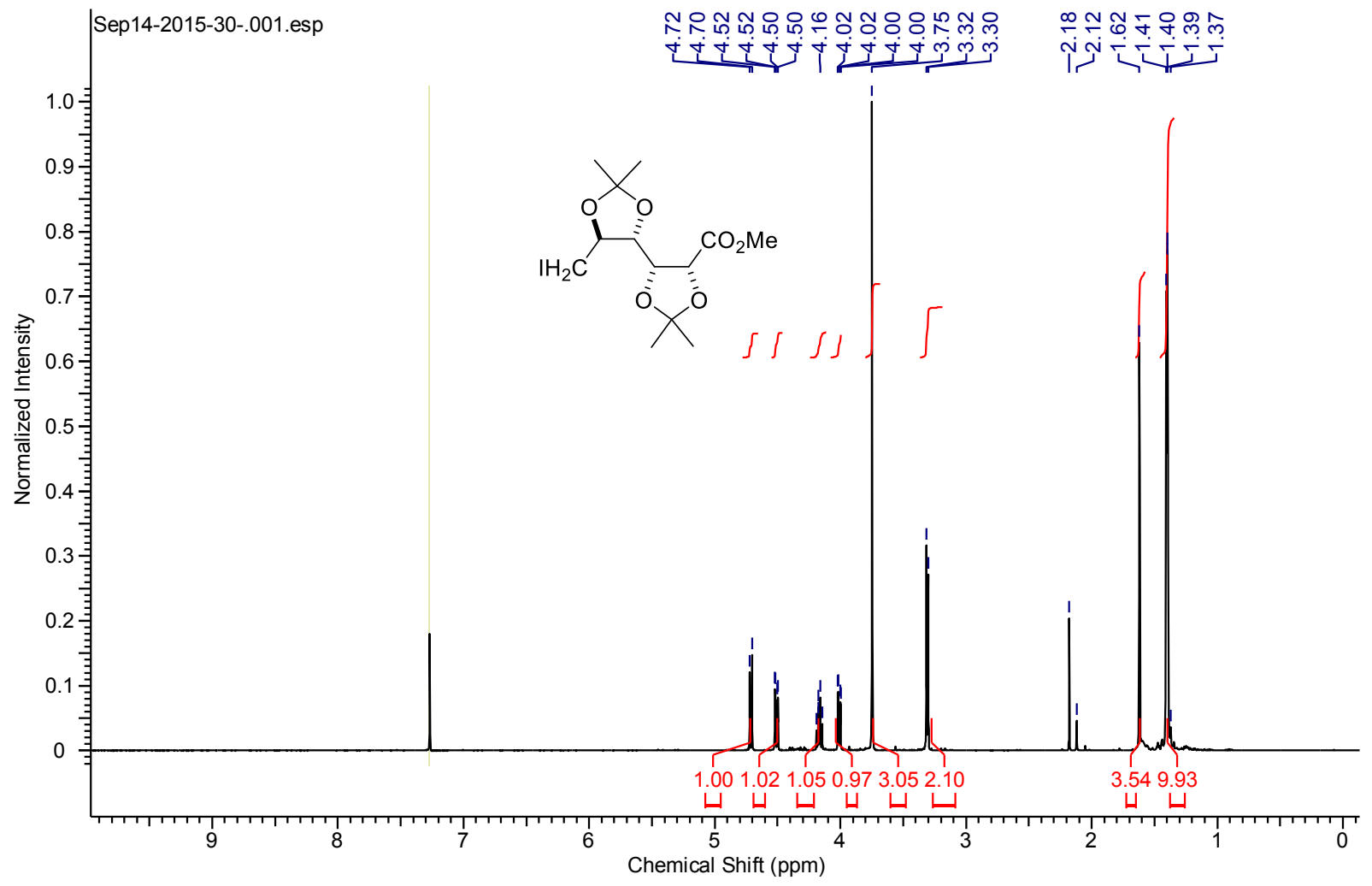


${ }^{13} \mathrm{C}$ NMR spectrum for $\mathbf{1 3}\left(\mathrm{CDCl}_{3}, 100 \mathrm{MHz}\right)$

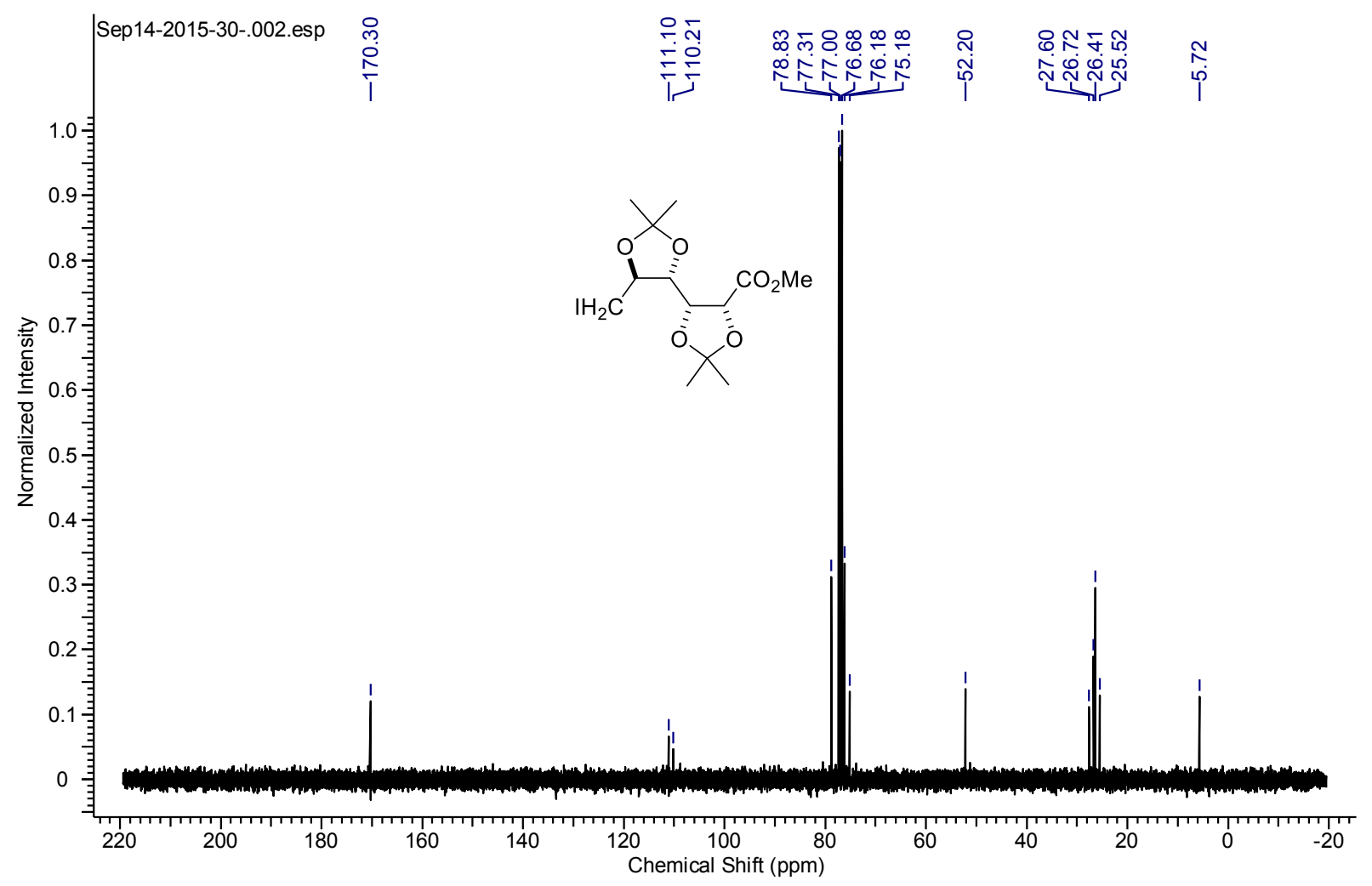


${ }^{1} \mathrm{H}$ NMR spectrum for $\mathbf{1 4}\left(\mathrm{CDCl}_{3}, 400 \mathrm{MHz}\right)$

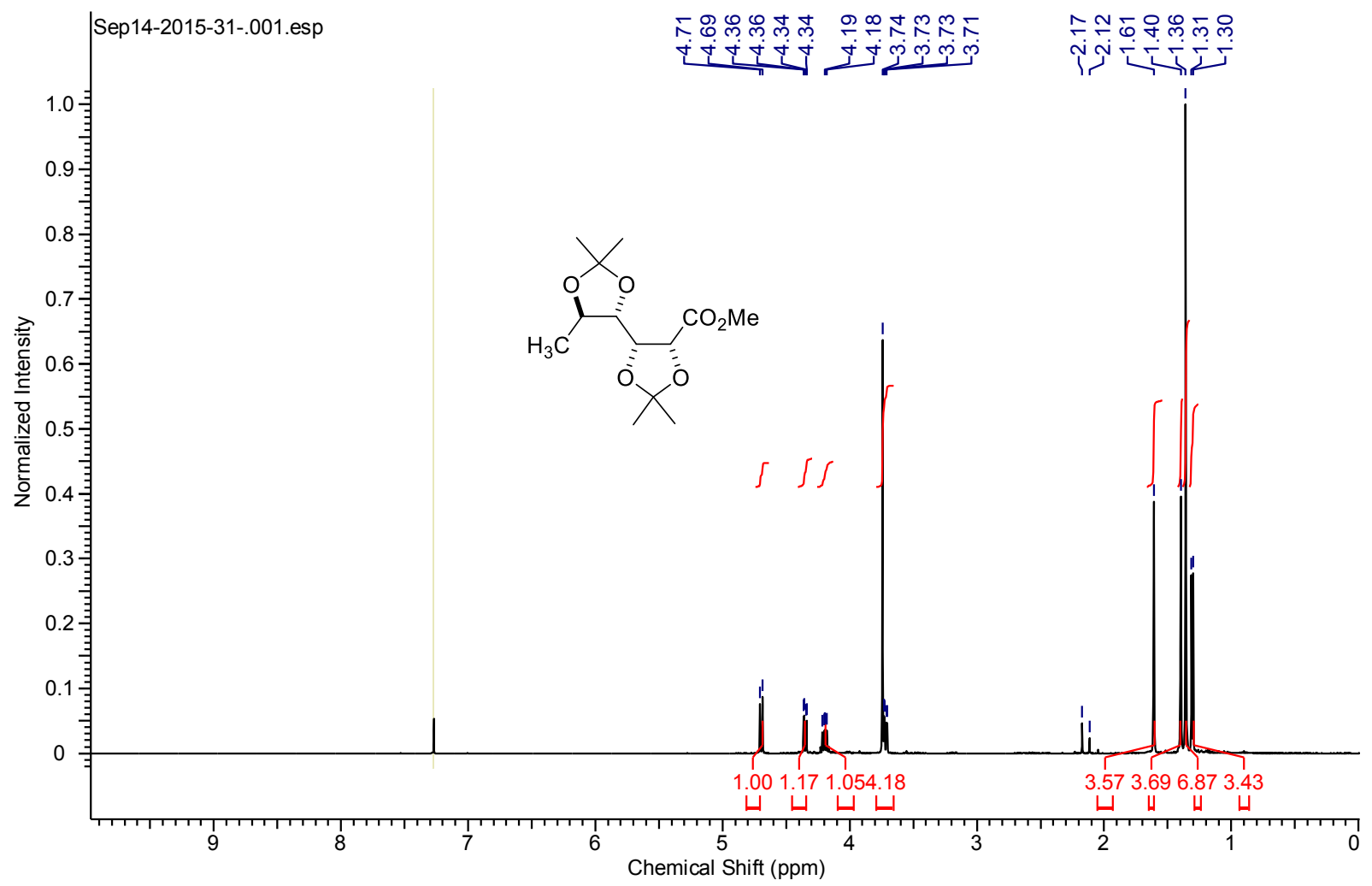


${ }^{13} \mathrm{C}$ NMR spectrum for $\mathbf{1 4}\left(\mathrm{CDCl}_{3}, 100 \mathrm{MHz}\right)$

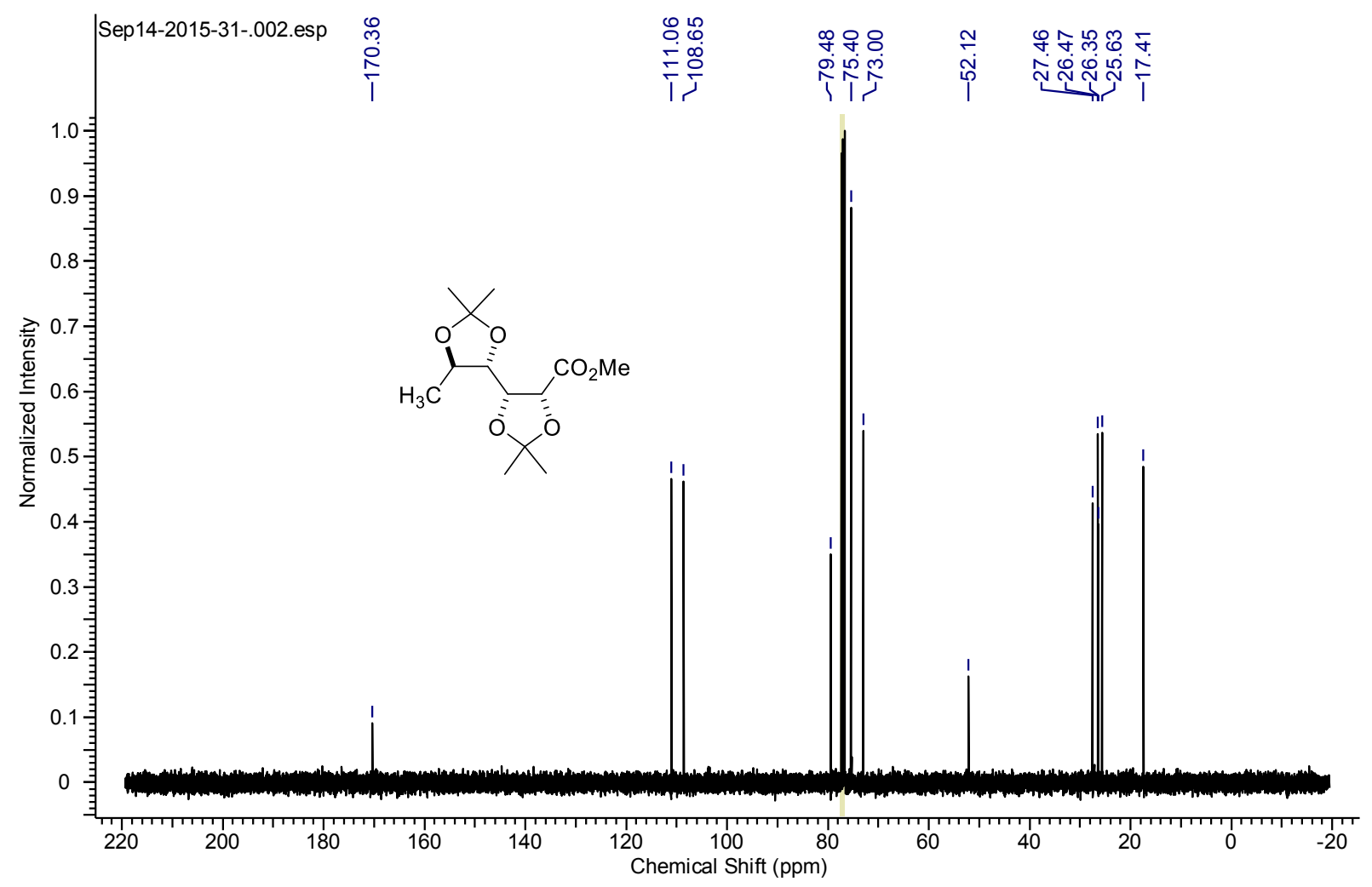


${ }^{1} \mathrm{H}$ NMR spectrum for $\mathbf{1 5}\left(\mathrm{D}_{2} \mathrm{O}, 400 \mathrm{MHz}\right)$

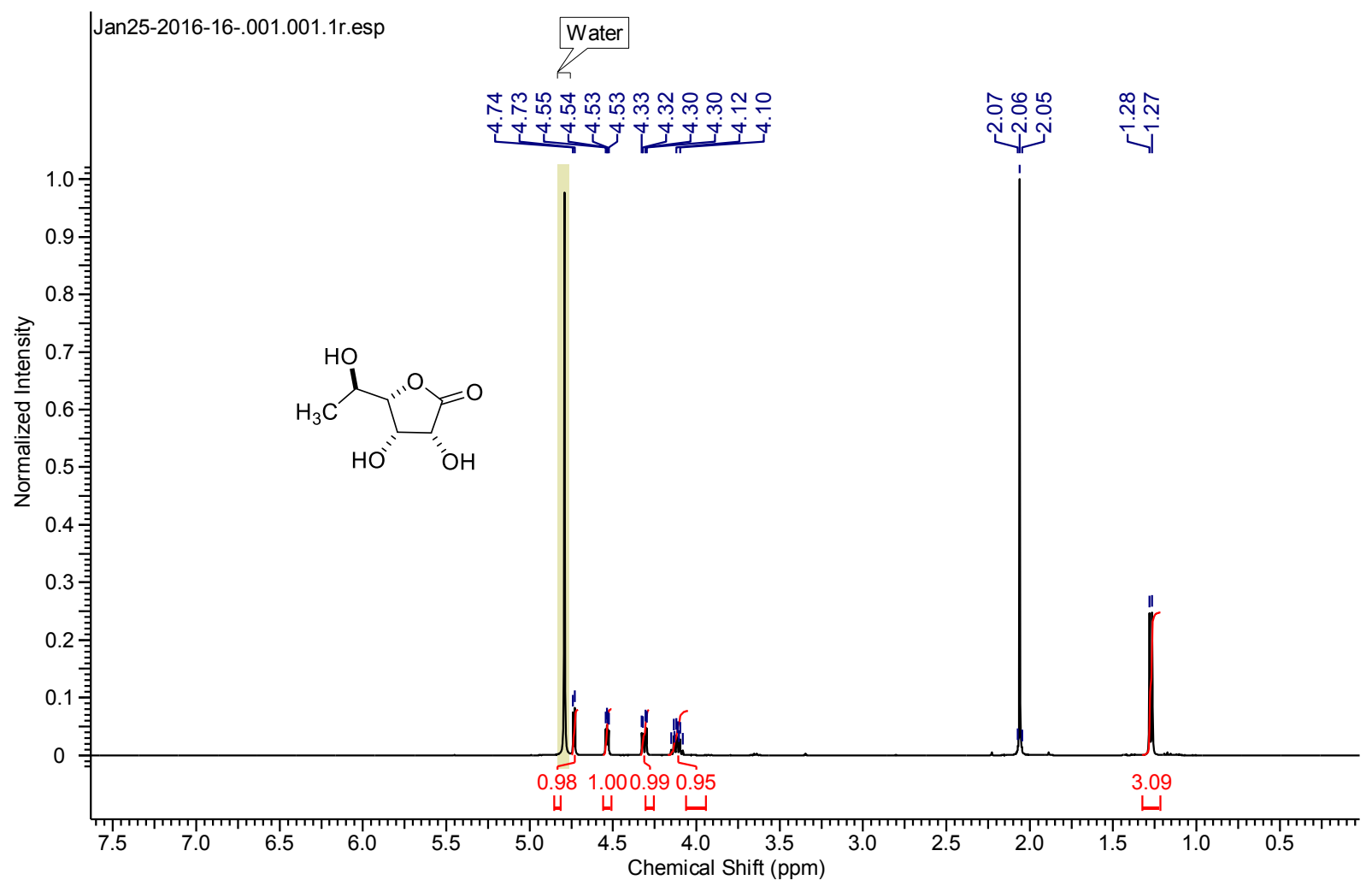


${ }^{13} \mathrm{C}$ NMR spectrum for $\mathbf{1 5}\left(\mathrm{D}_{2} \mathrm{O}, 100 \mathrm{MHz}\right)$

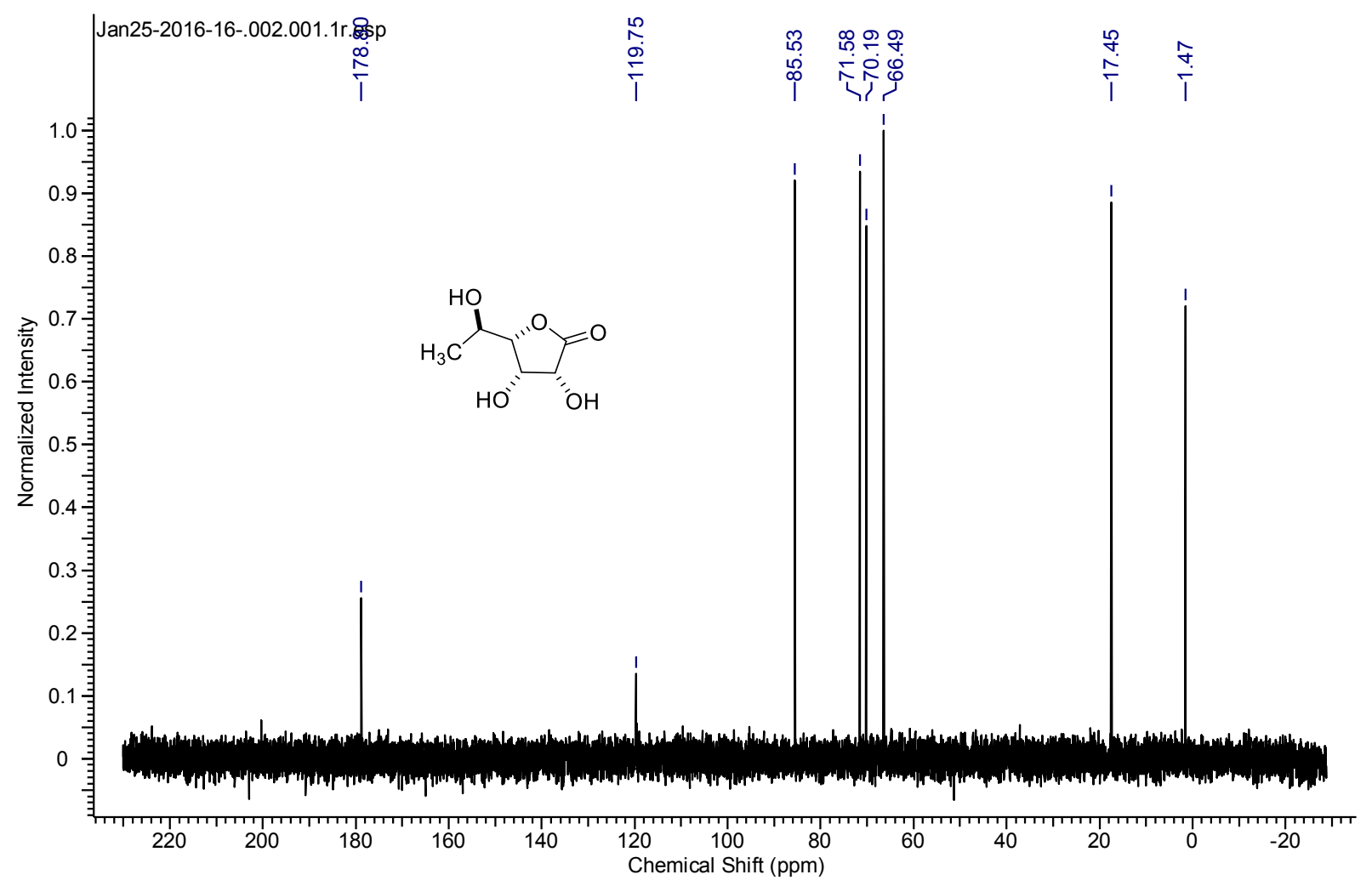


${ }^{1} \mathrm{H}$ NMR spectrum for $\mathbf{1 6}\left(\mathrm{CDCl}_{3}, 400 \mathrm{MHz}\right)$

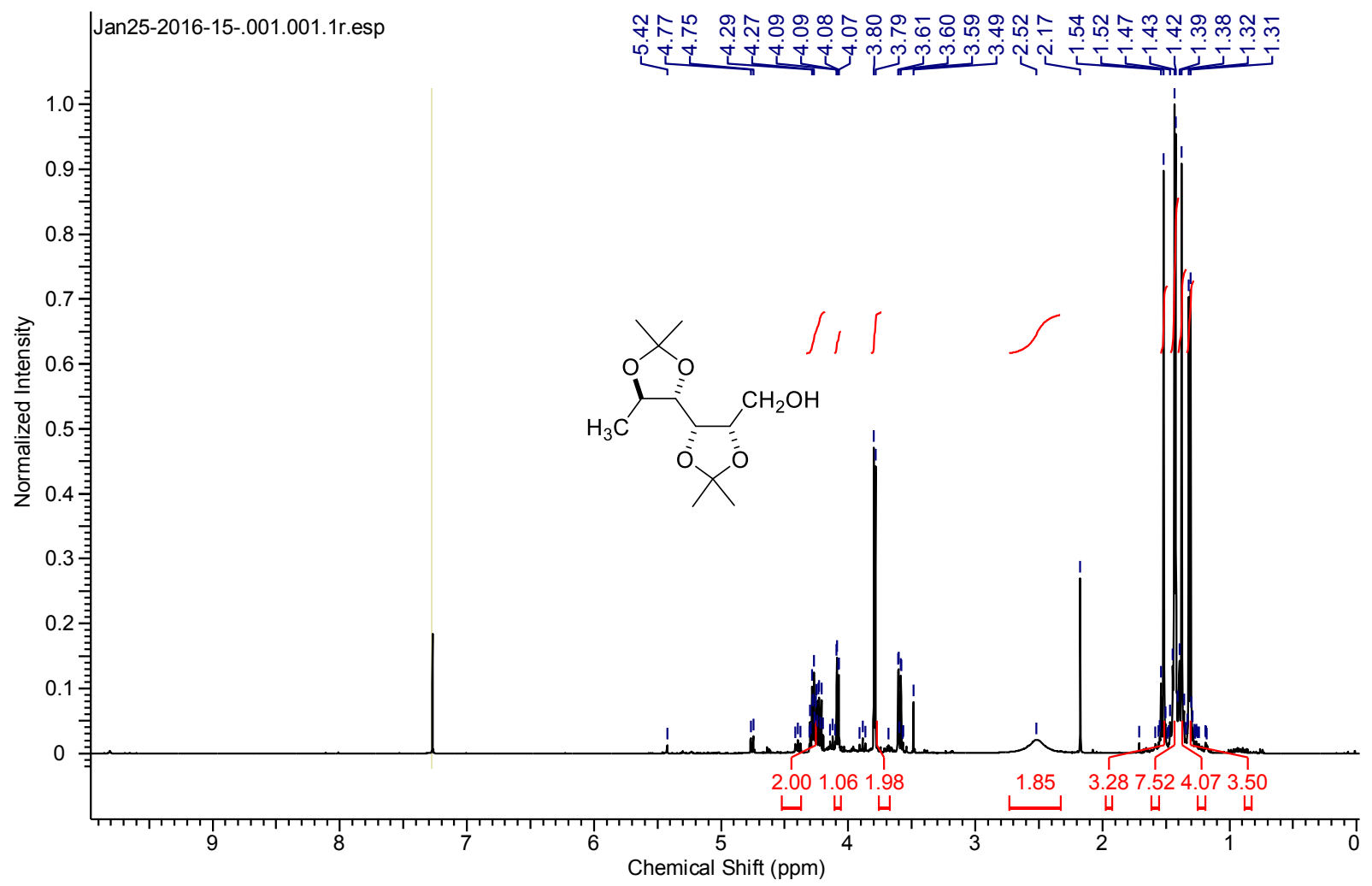


${ }^{13} \mathrm{C}$ NMR spectrum for $\mathbf{1 6}\left(\mathrm{CDCl}_{3}, 100 \mathrm{MHz}\right)$

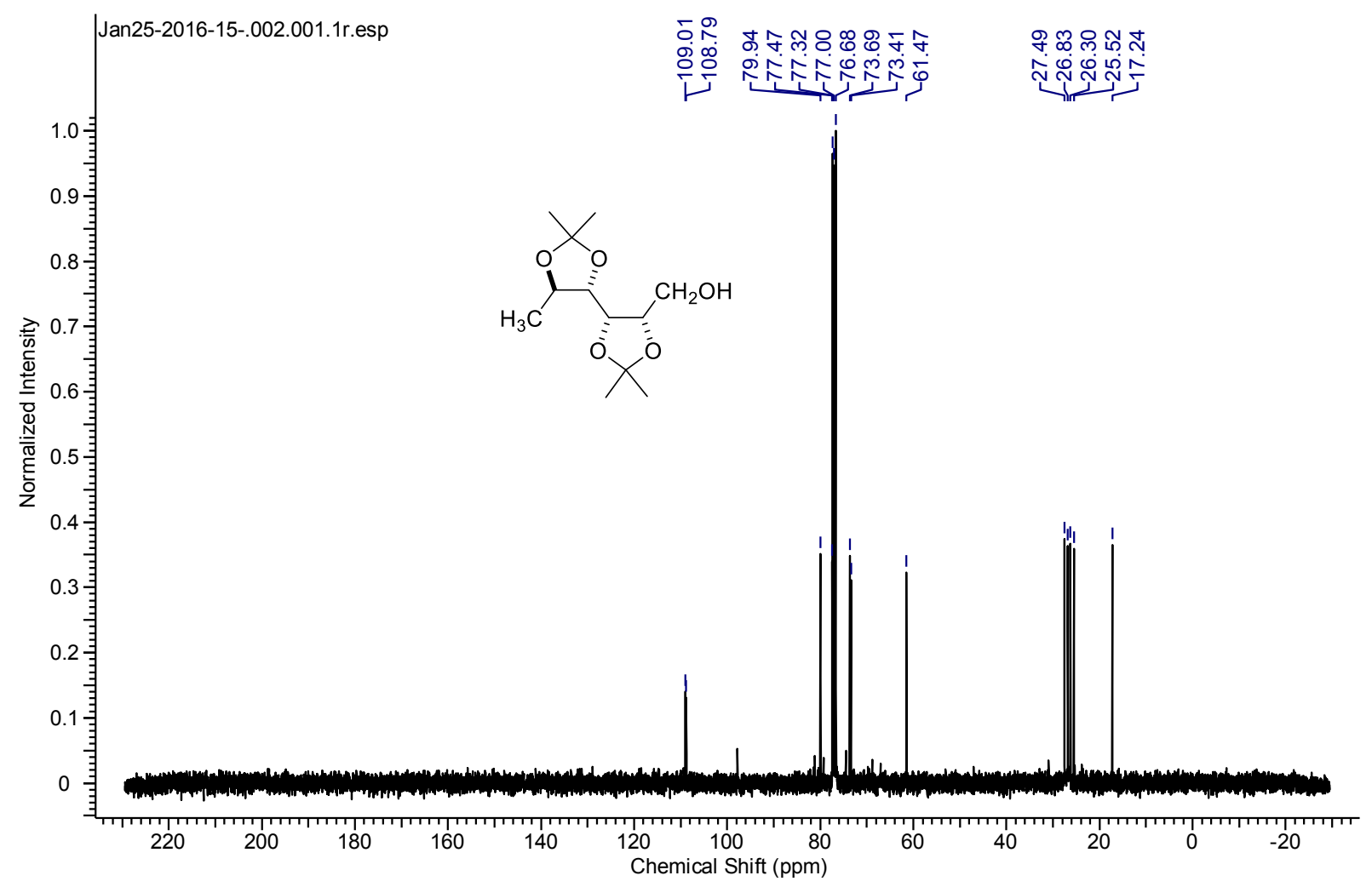


${ }^{1} \mathrm{H}$ NMR spectrum for $\mathbf{1 7}\left(\mathrm{CD}_{3} \mathrm{CN}, 400 \mathrm{MHz}\right)$

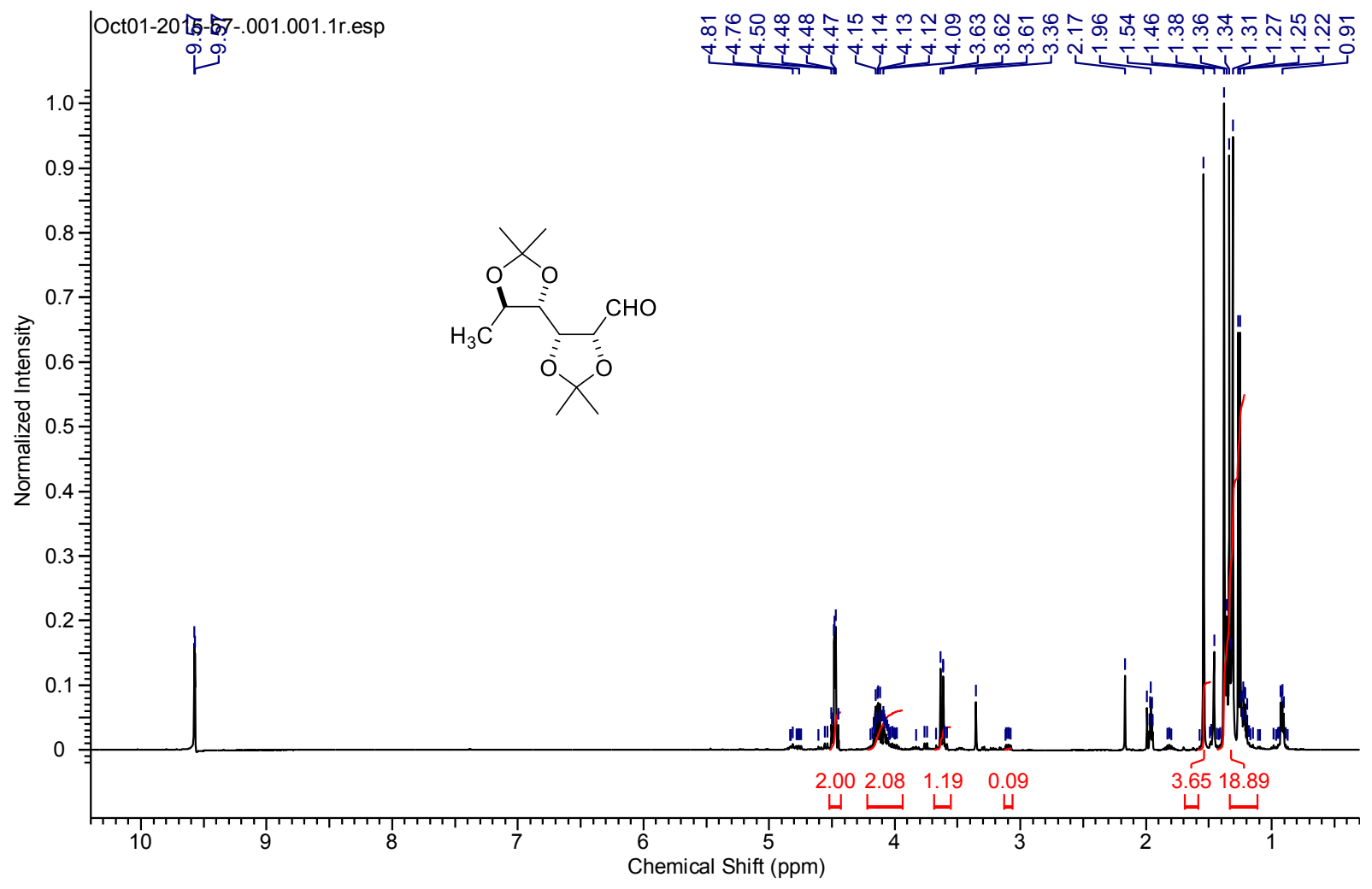


${ }^{13} \mathrm{C}$ NMR spectrum for $\mathbf{1 7}\left(\mathrm{CD}_{3} \mathrm{CN}, 100 \mathrm{MHz}\right)$

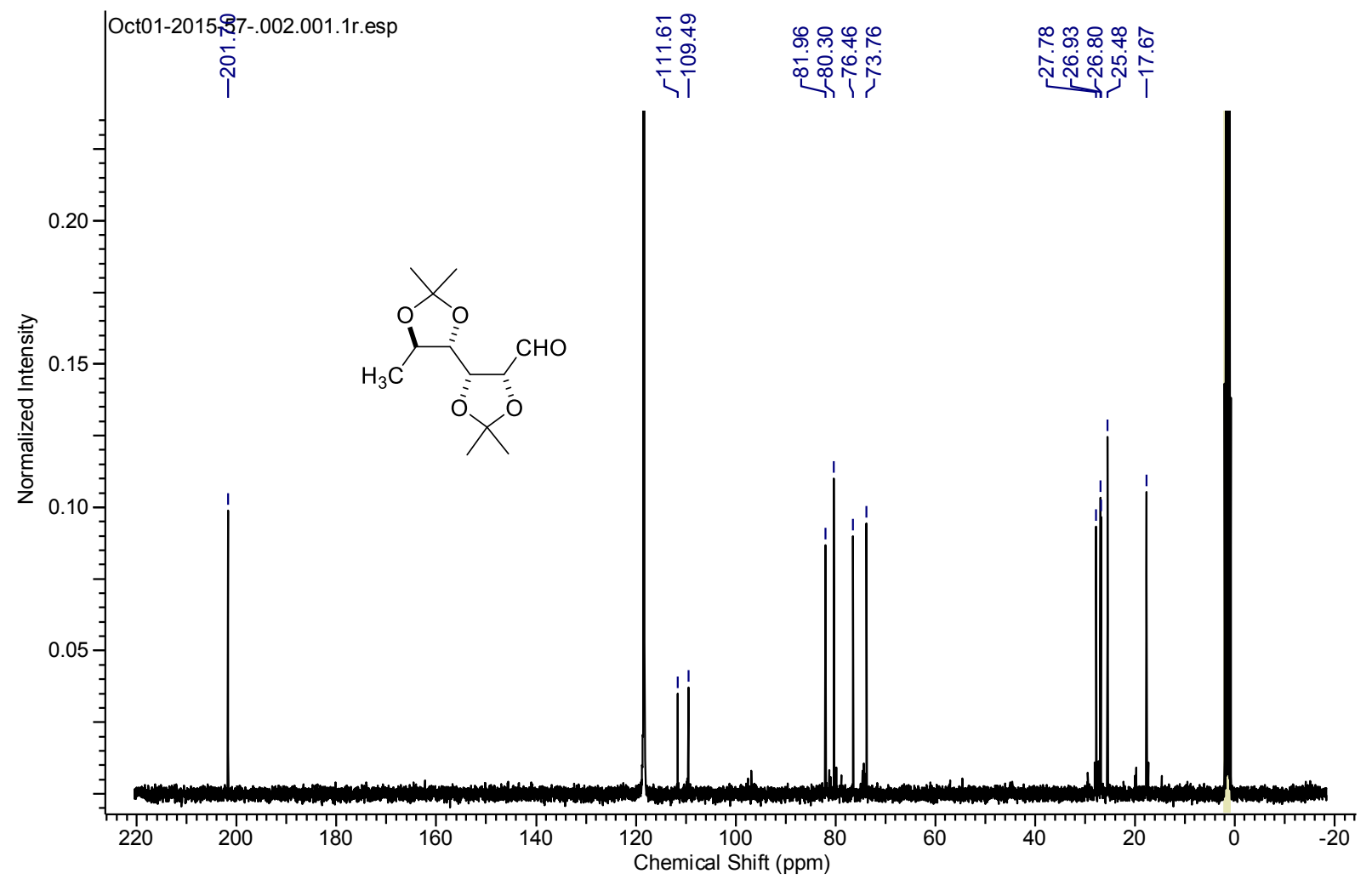


${ }^{1} \mathrm{H}$ NMR spectrum for $\mathbf{1 8}\left(\mathrm{D}_{2} \mathrm{O}, 400 \mathrm{MHz}\right)$

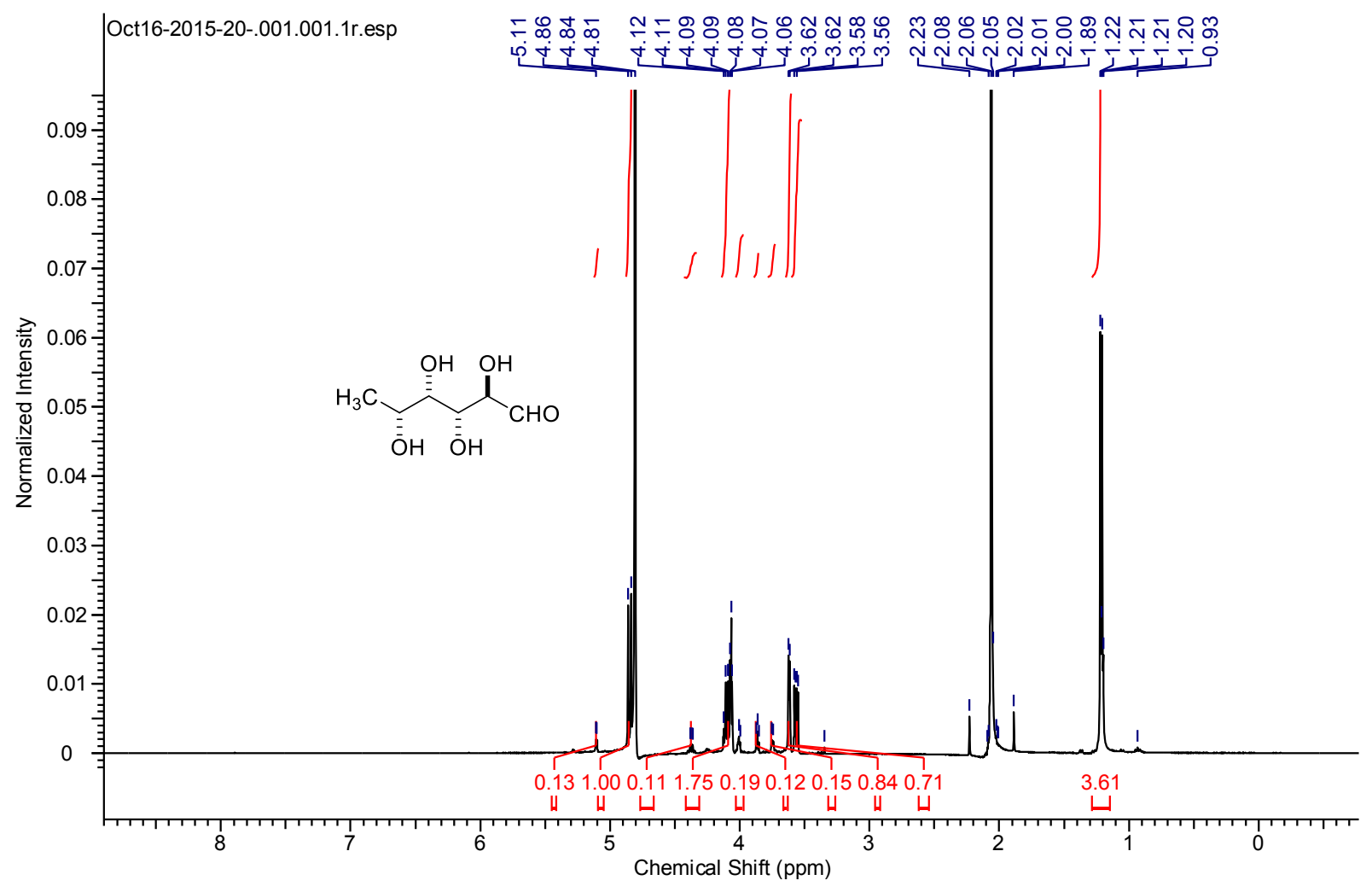


${ }^{13} \mathrm{C}$ NMR spectrum for $\mathbf{1 8}\left(\mathrm{D}_{2} \mathrm{O}, 100 \mathrm{MHz}\right)$

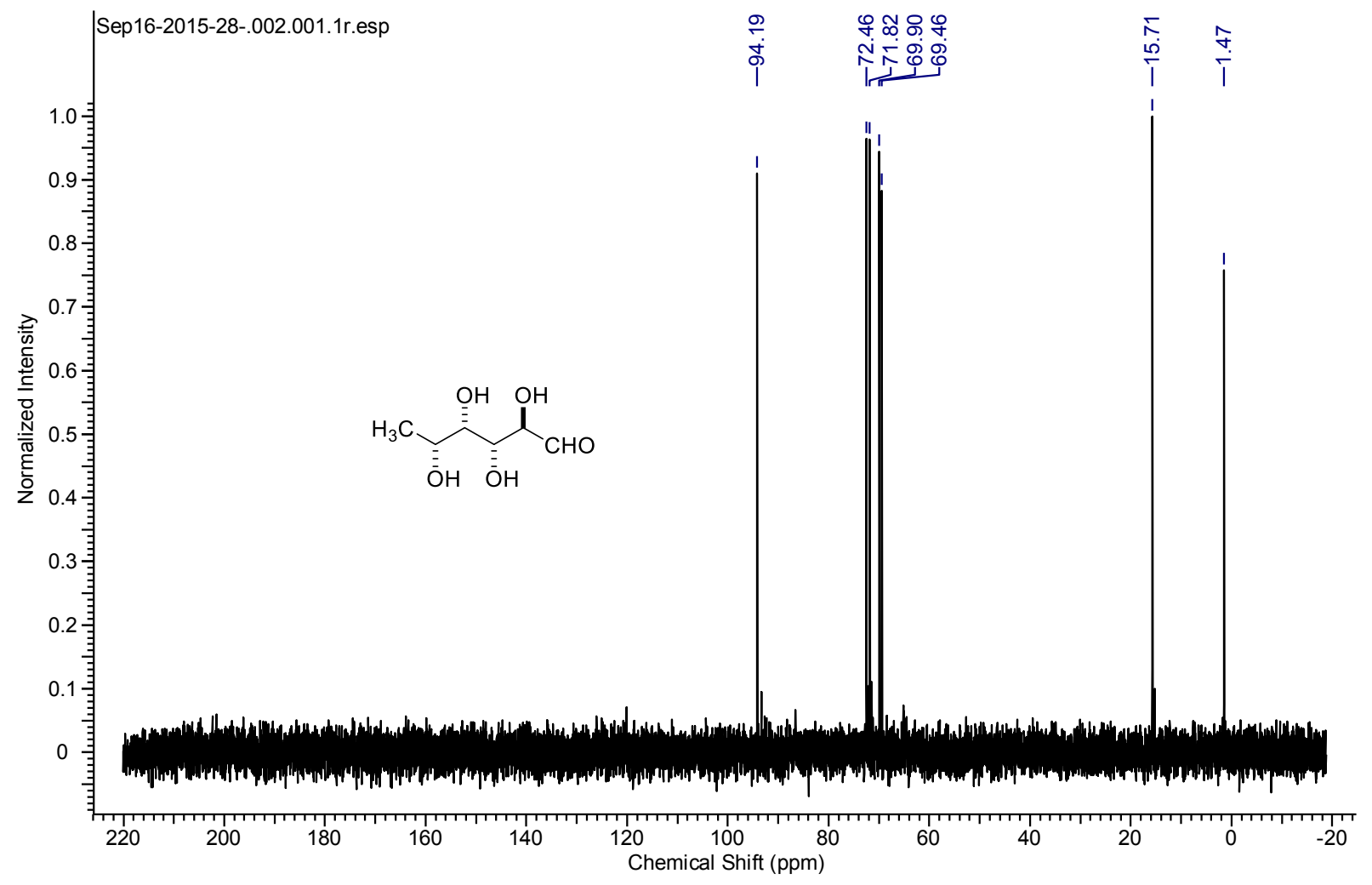


${ }^{1} \mathrm{H}$ NMR spectrum for $19\left(\mathrm{D}_{2} \mathrm{O}, 400 \mathrm{MHz}\right)$

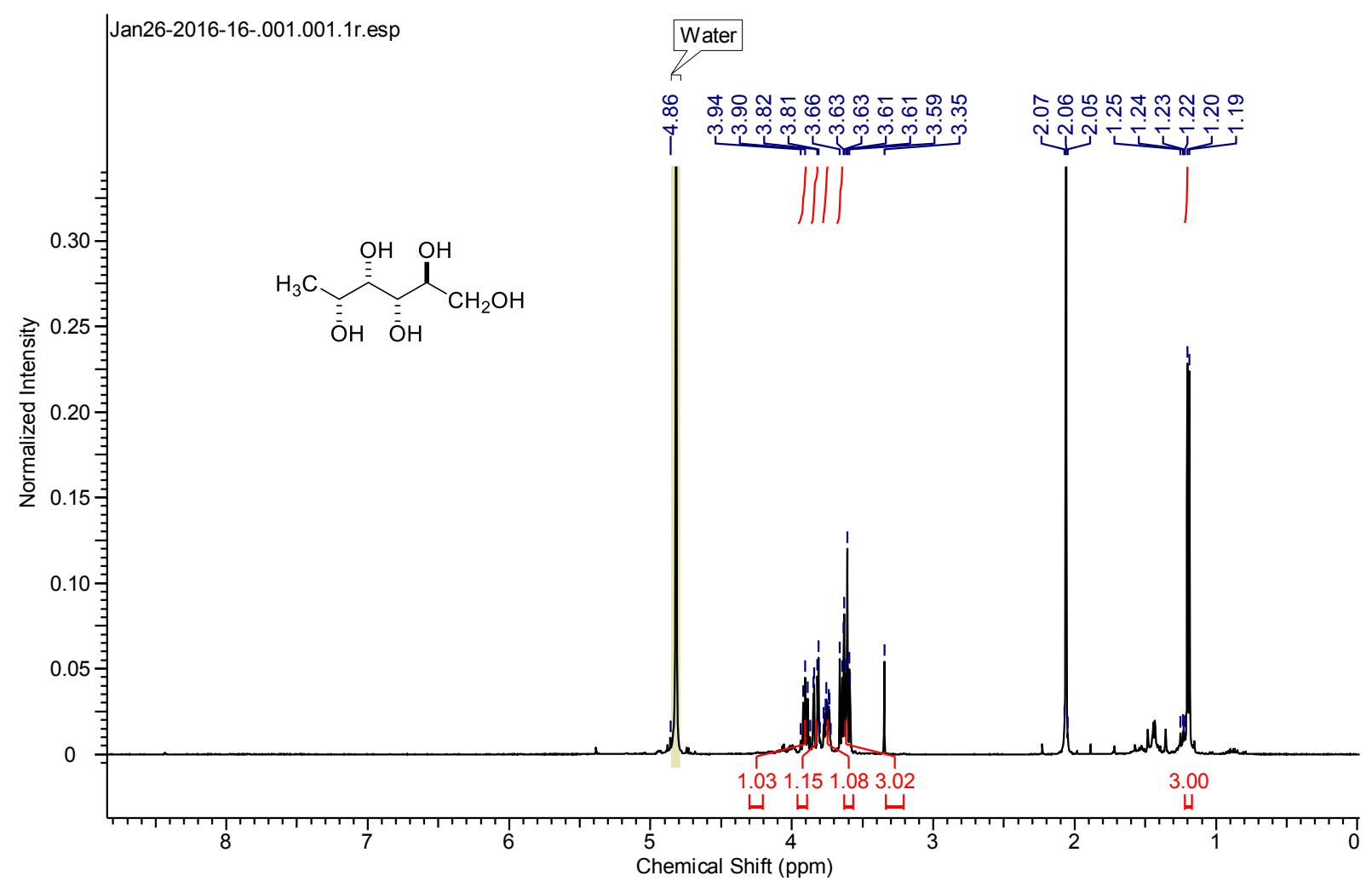


${ }^{13} \mathrm{C}$ NMR spectrum for $19\left(\mathrm{D}_{2} \mathrm{O}, 100 \mathrm{MHz}\right)$

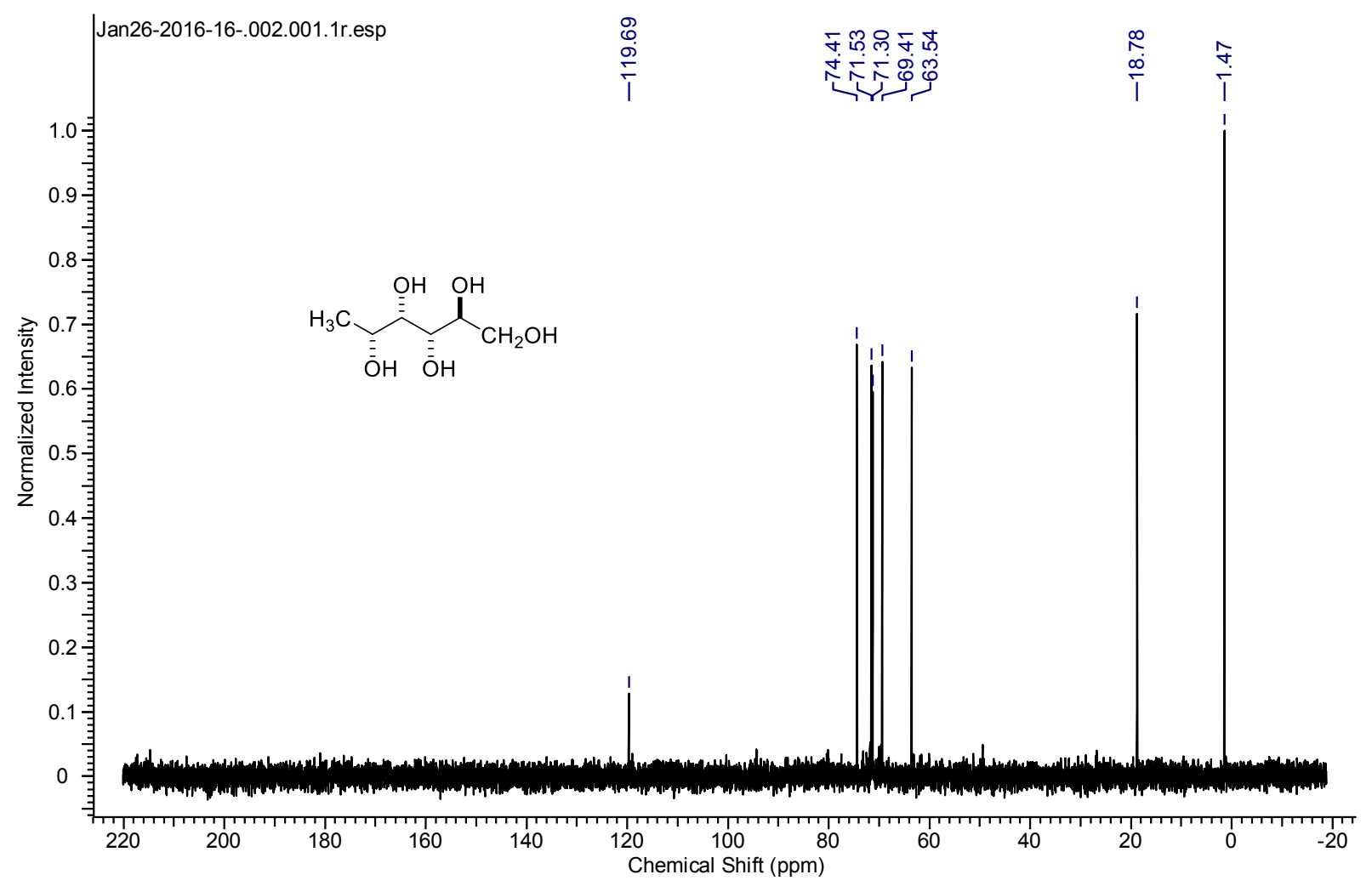


${ }^{1} \mathrm{H}$ NMR spectrum for $\mathbf{2 0}\left(\mathrm{CDCl}_{3}, 400 \mathrm{MHz}\right)$

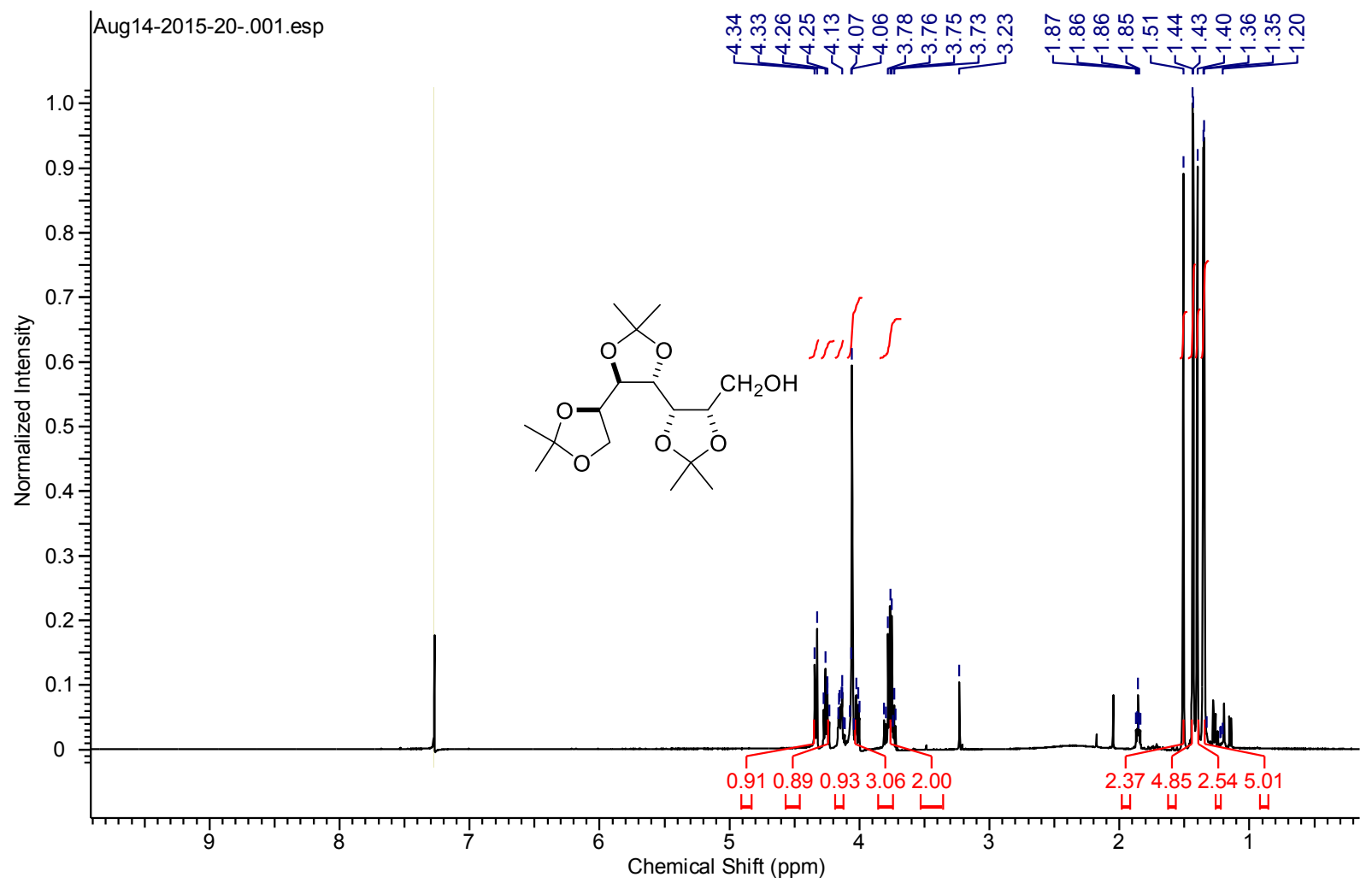


${ }^{13} \mathrm{C}$ NMR spectrum for $20\left(\mathrm{CDCl}_{3}, 100 \mathrm{MHz}\right)$

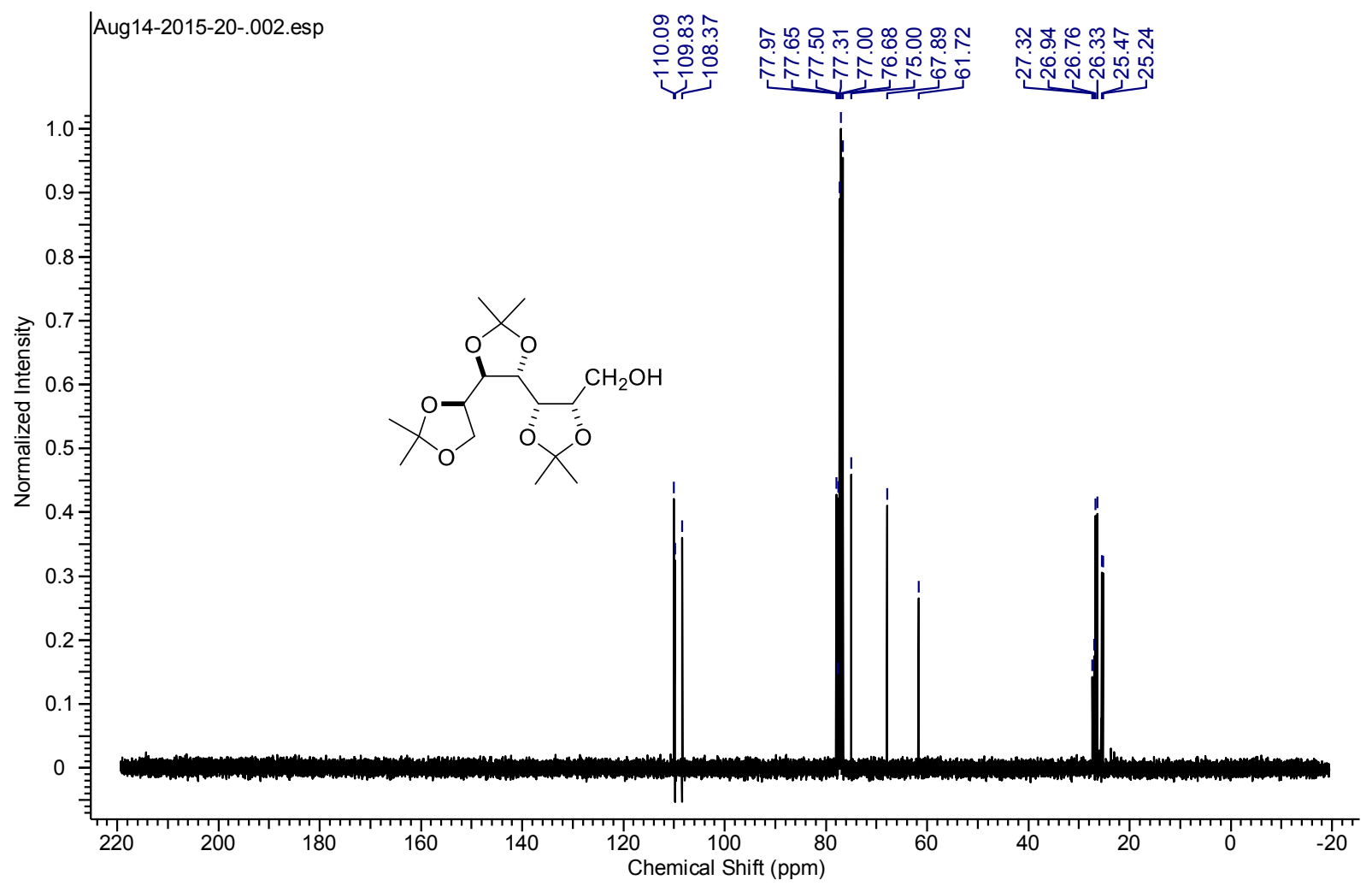


${ }^{1} \mathrm{H}$ NMR spectrum for $\mathbf{2 1}\left(\mathrm{D}_{2} \mathrm{O}, 400 \mathrm{MHz}\right)$

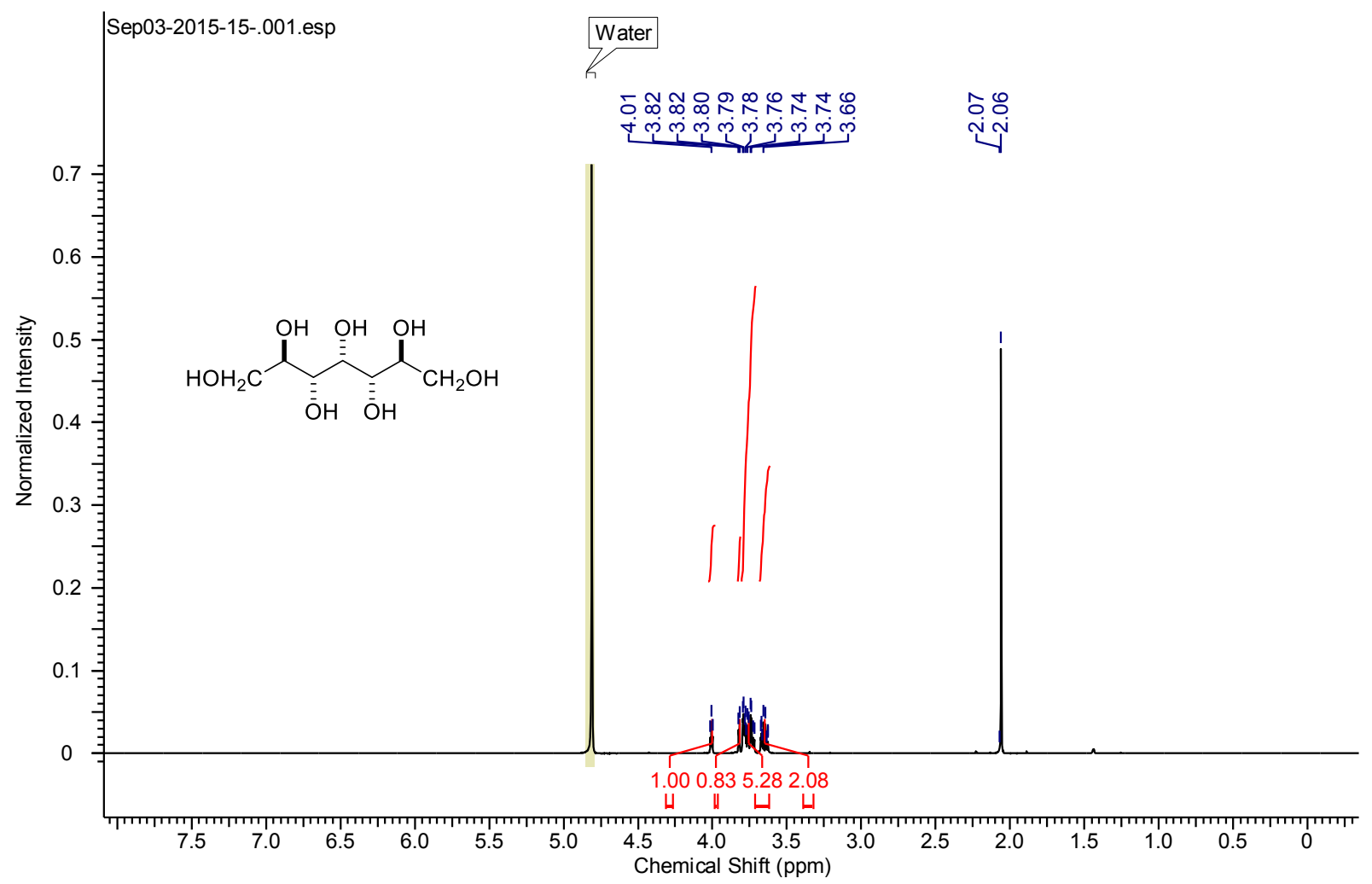


${ }^{13} \mathrm{C}$ NMR spectrum for $\mathbf{2 1}\left(\mathrm{D}_{2} \mathrm{O}, 100 \mathrm{MHz}\right)$

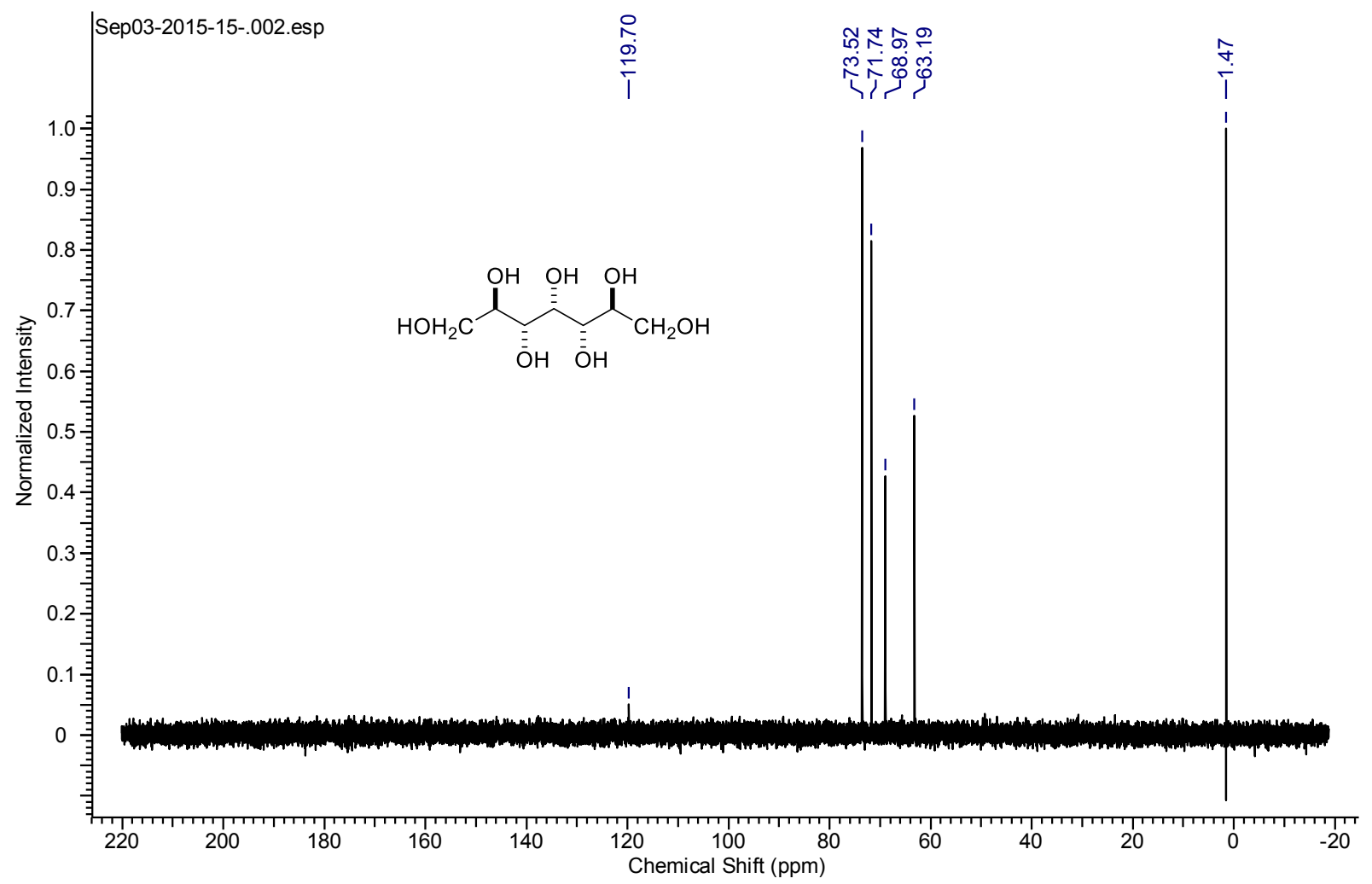


${ }^{1} \mathrm{H}$ NMR spectrum for $23\left(\mathrm{CD}_{3} \mathrm{CN}, 500 \mathrm{MHz}\right)$

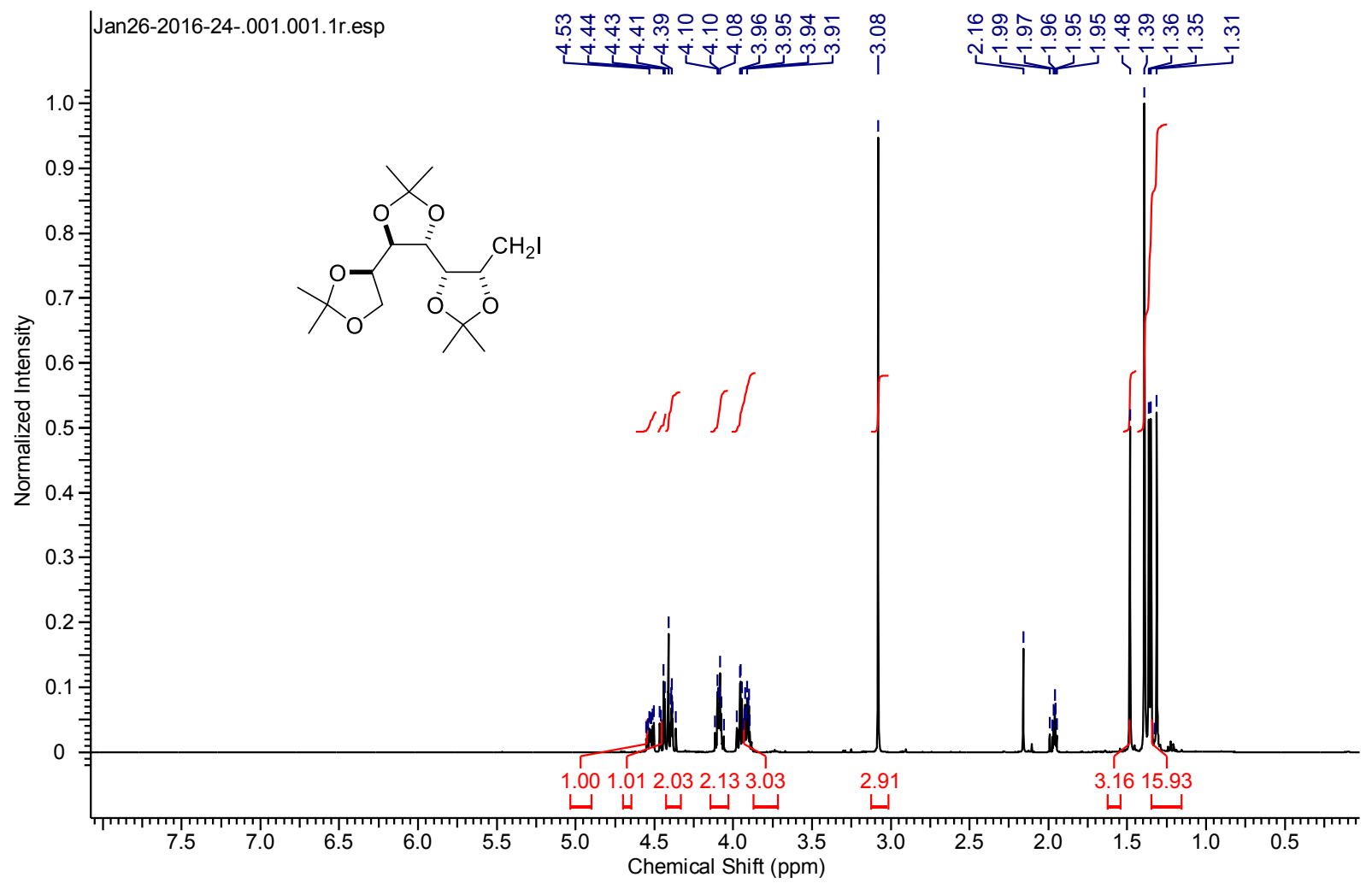


${ }^{13} \mathrm{C}$ NMR spectrum for $23\left(\mathrm{CD}_{3} \mathrm{CN}, 100 \mathrm{MHz}\right)$

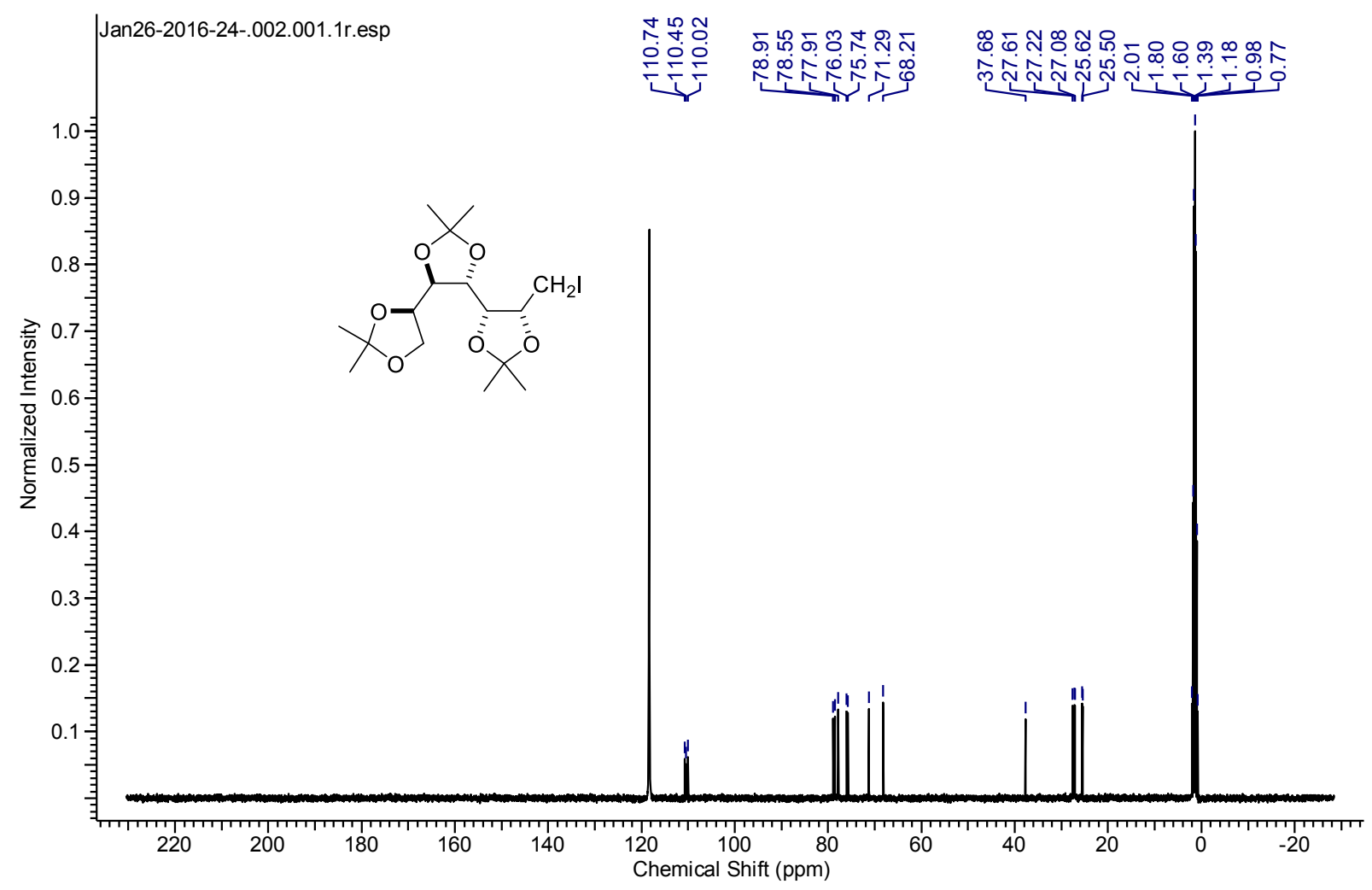


${ }^{1} \mathrm{H}$ NMR spectrum for $\mathbf{2 6}\left(\mathrm{CDCl}_{3}, 400 \mathrm{MHz}\right)$

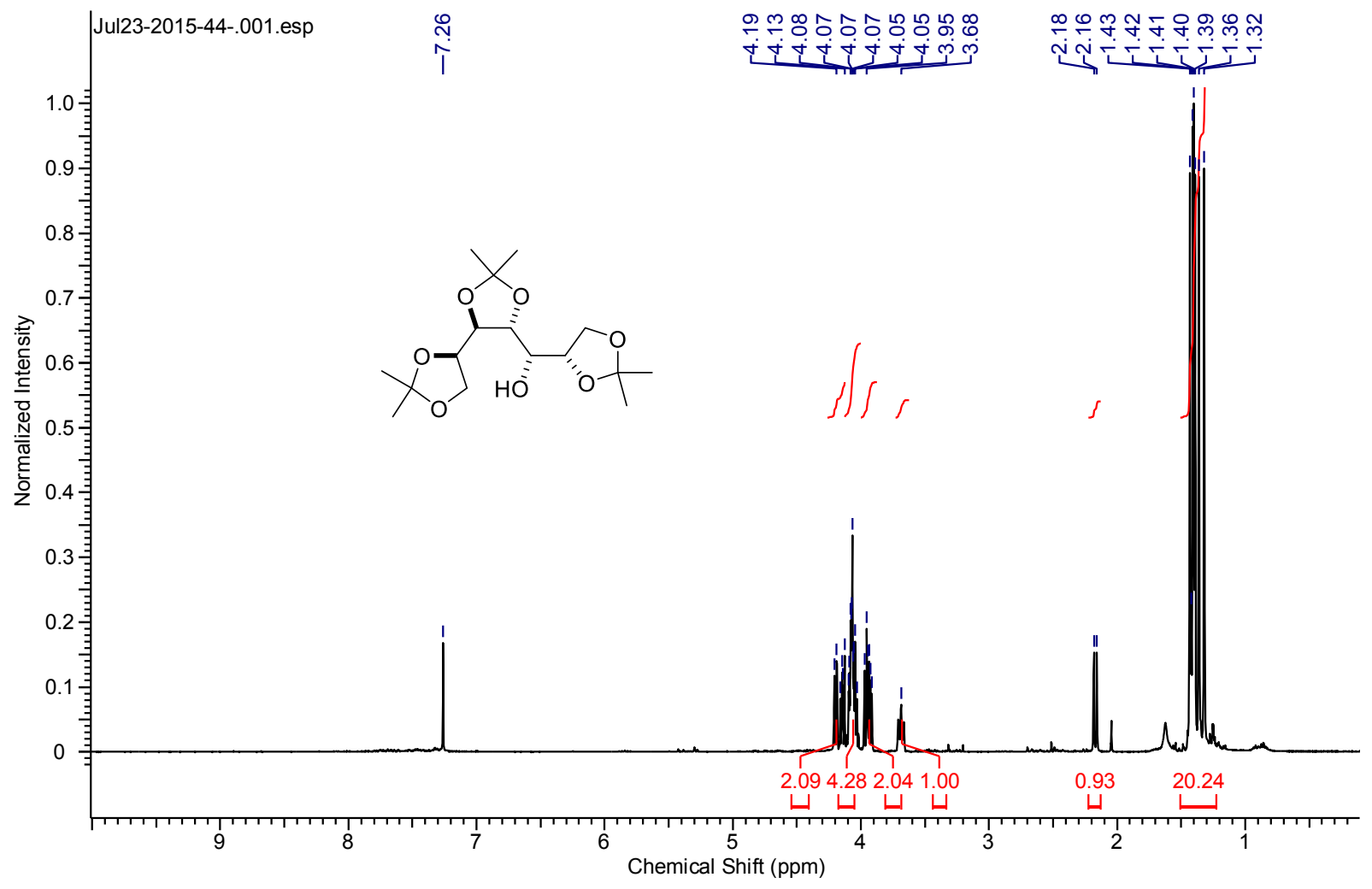


${ }^{13} \mathrm{C}$ NMR spectrum for $26\left(\mathrm{CDCl}_{3}, 100 \mathrm{MHz}\right)$

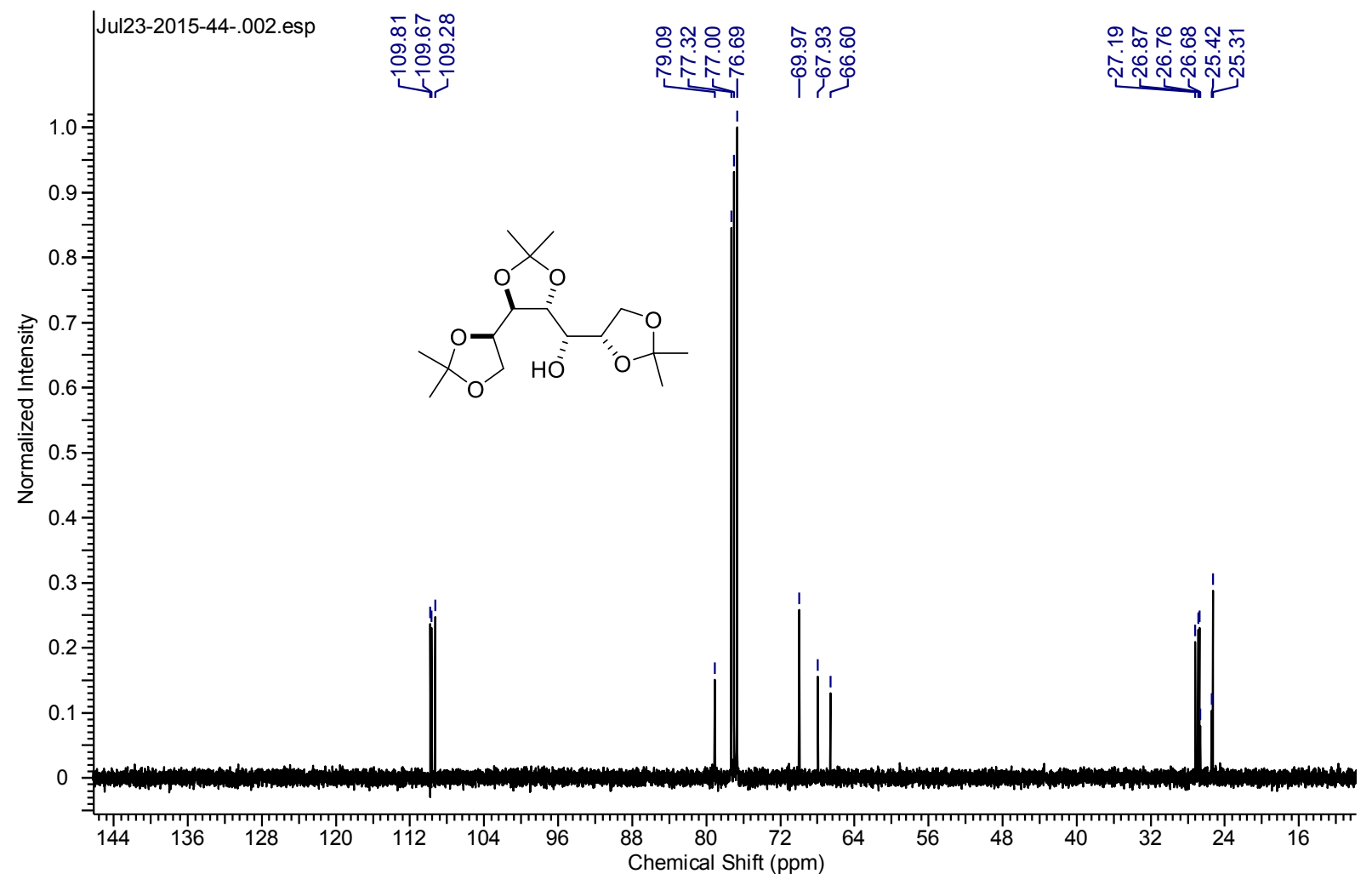


${ }^{1} \mathrm{H}$ NMR spectrum for $25\left(\mathrm{CDCl}_{3}, 400 \mathrm{MHz}\right)$

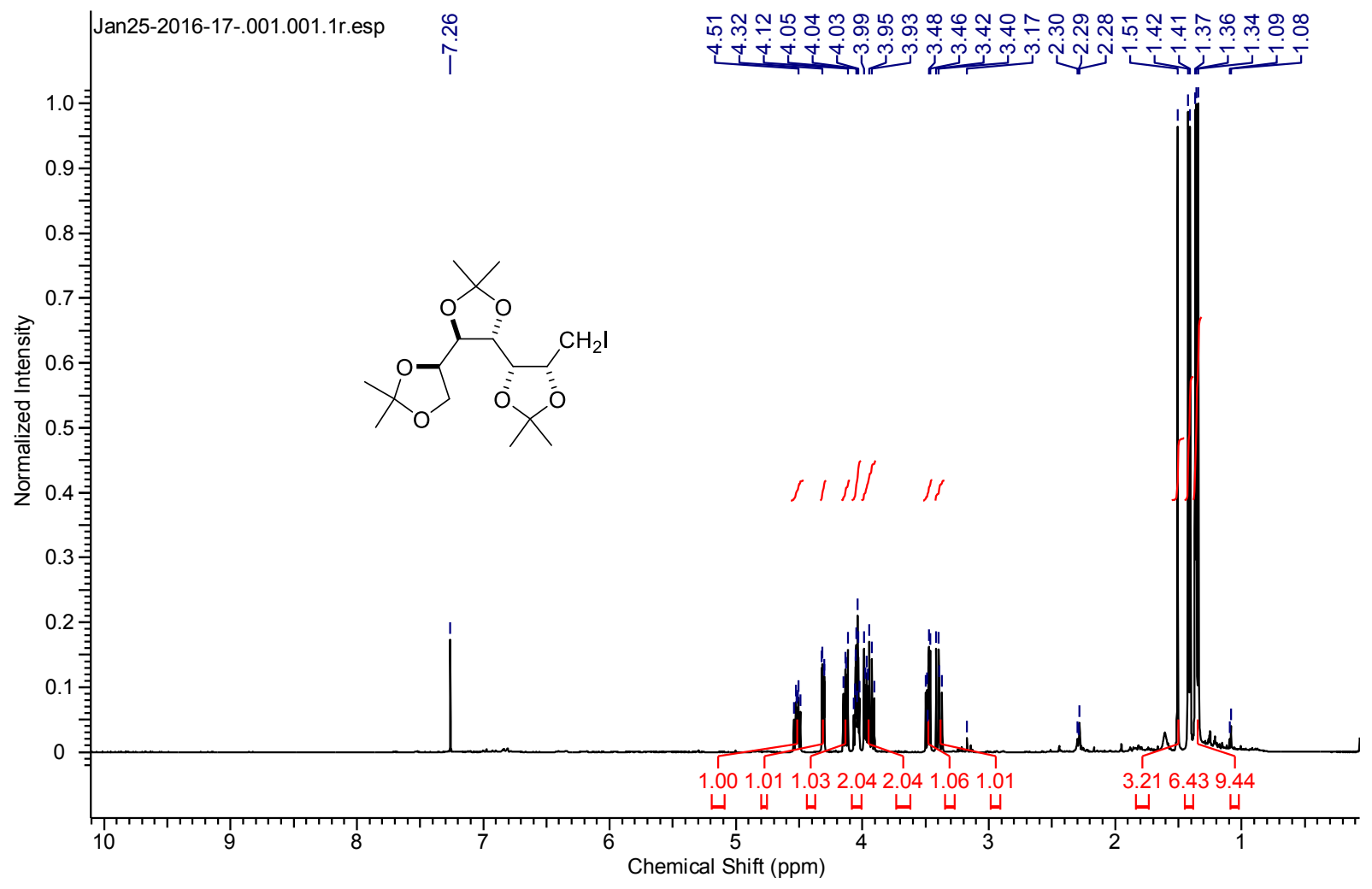


${ }^{13} \mathrm{C}$ NMR spectrum for $25\left(\mathrm{CDCl}_{3}, 100 \mathrm{MHz}\right)$

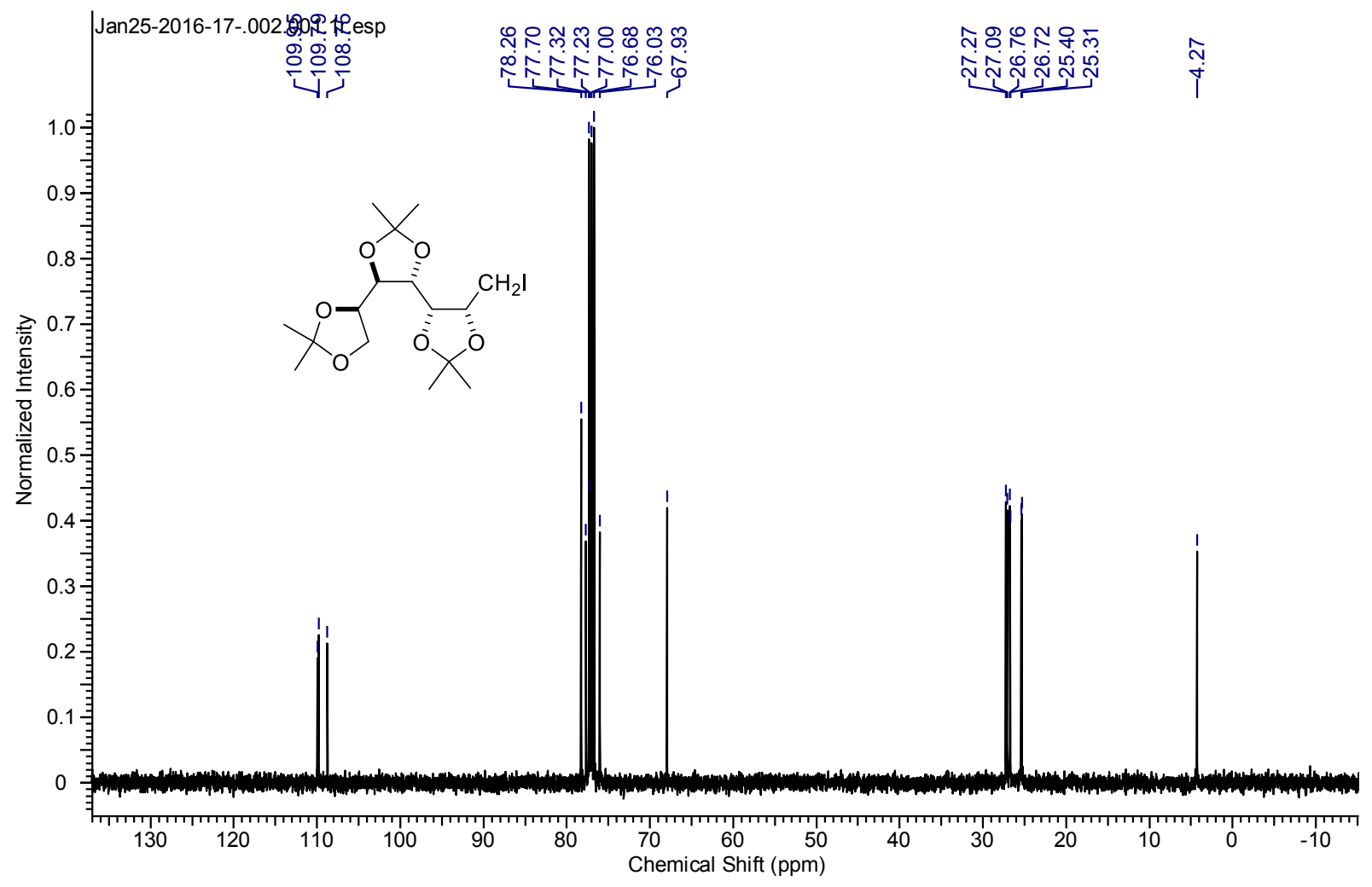


${ }^{1} \mathrm{H}$ NMR spectrum for $\mathbf{2 7}\left(\mathrm{CDCl}_{3}, 400 \mathrm{MHz}\right)$

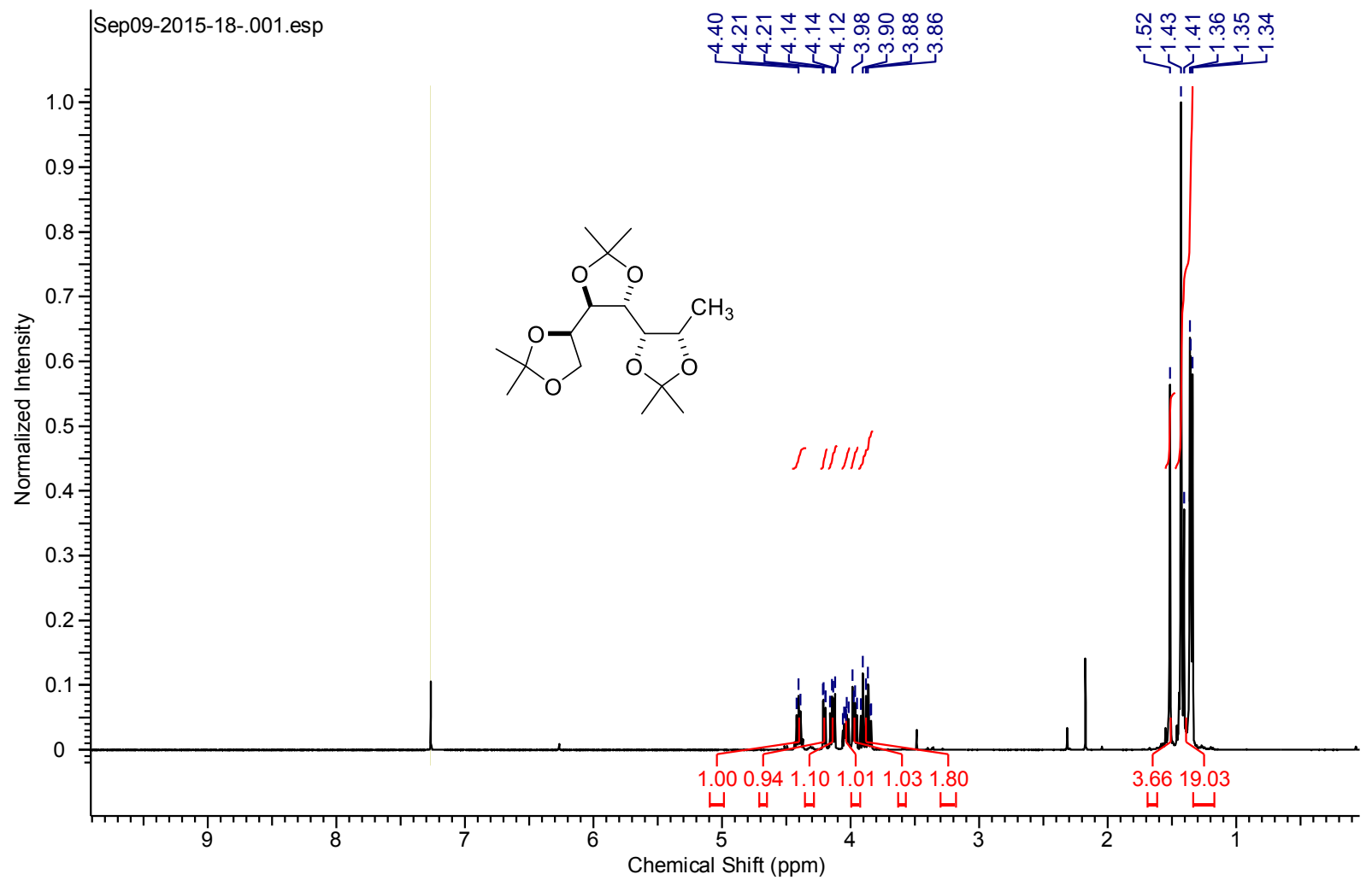




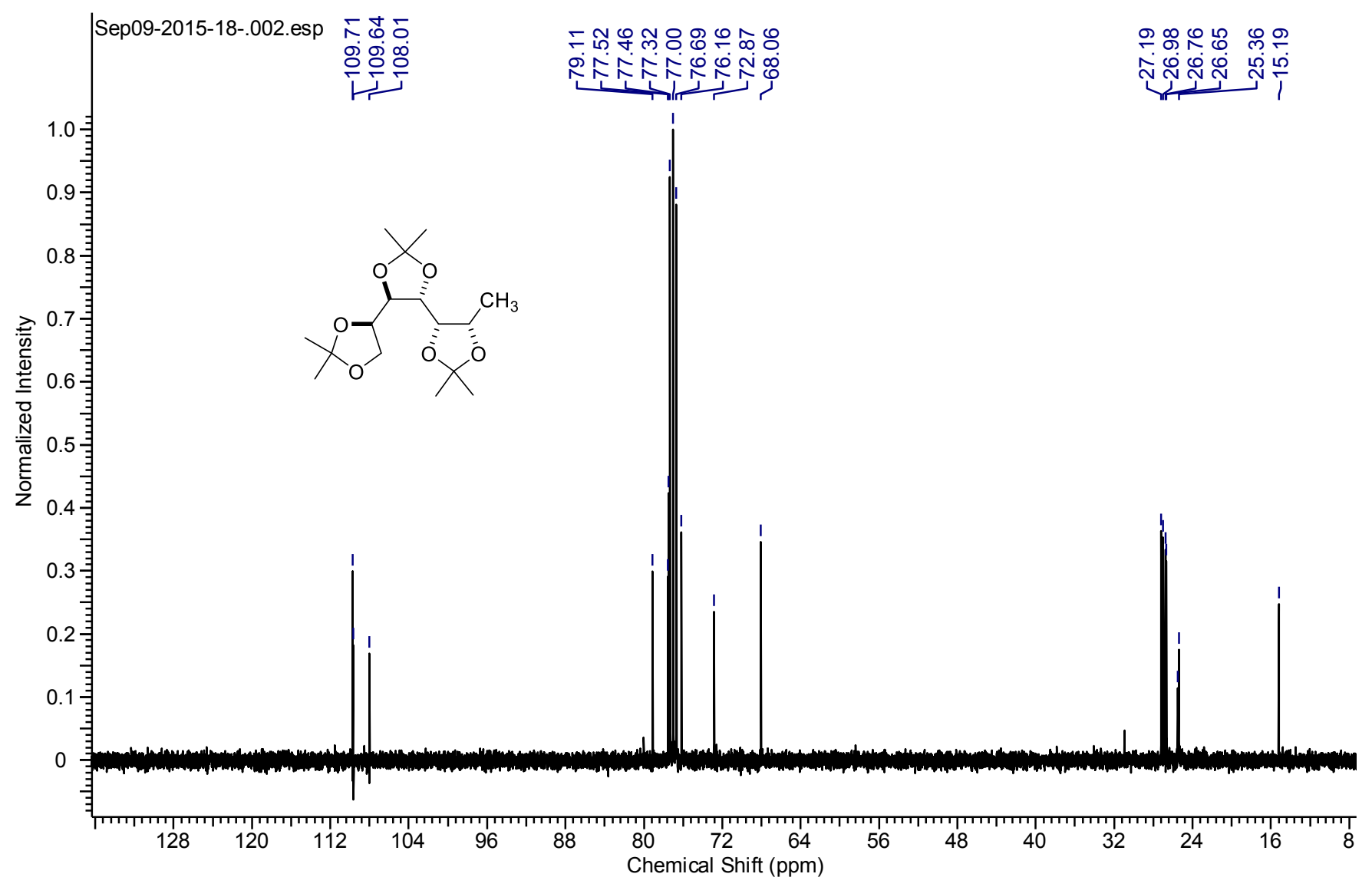


${ }^{1} \mathrm{H}$ NMR spectrum for $\mathbf{2 8}\left(\mathrm{CD}_{3} \mathrm{CN}, 400 \mathrm{MHz}\right)$

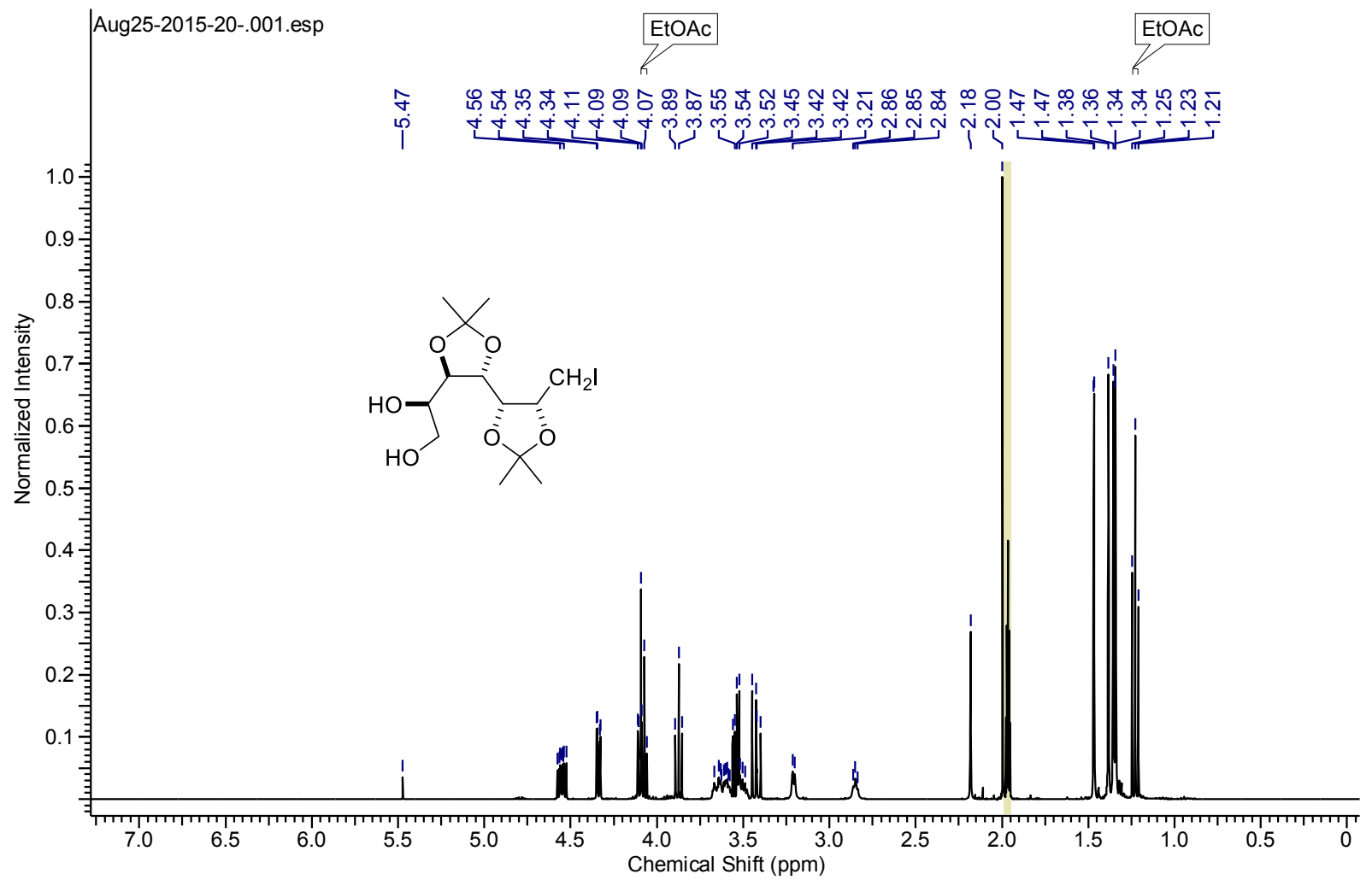


${ }^{13} \mathrm{C}$ NMR spectrum for $\mathbf{2 8}\left(\mathrm{CD}_{3} \mathrm{CN}, 100 \mathrm{MHz}\right)$

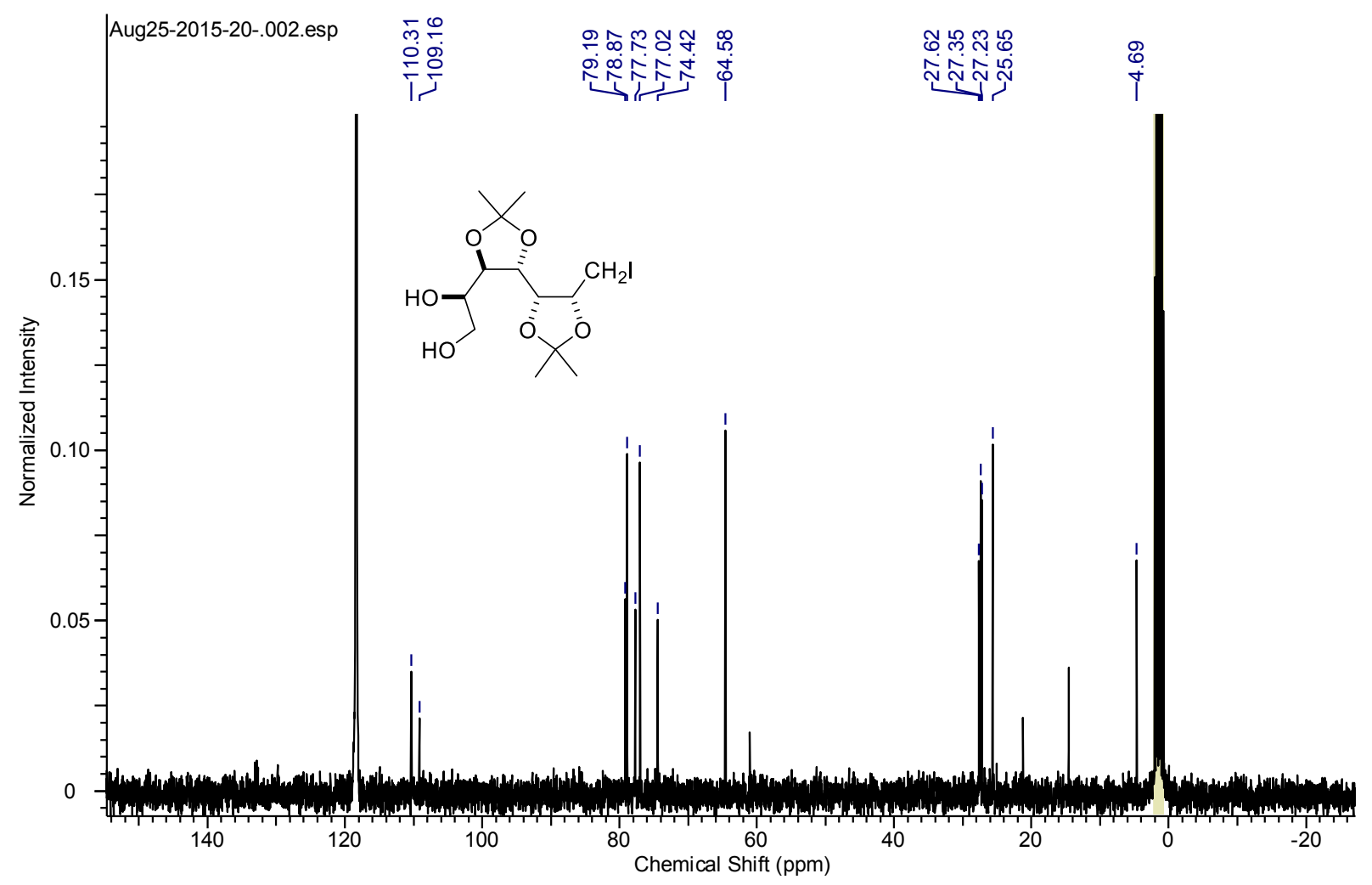


${ }^{1} \mathrm{H}$ NMR spectrum for $29\left(\mathrm{CD}_{3} \mathrm{OD}, 400 \mathrm{MHz}\right)$

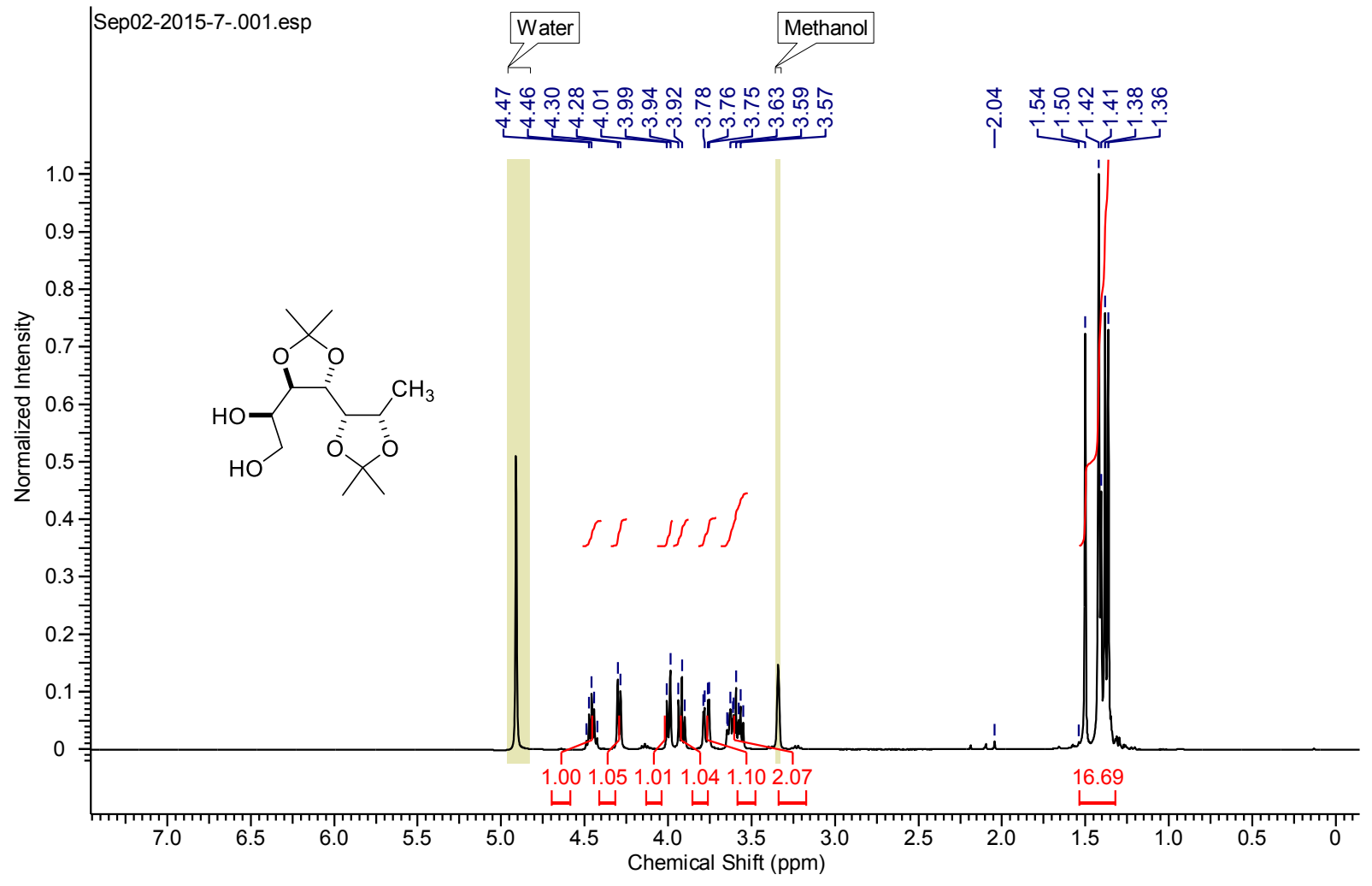


${ }^{13} \mathrm{C}$ NMR spectrum for $29\left(\mathrm{CD}_{3} \mathrm{OD}, 100 \mathrm{MHz}\right)$

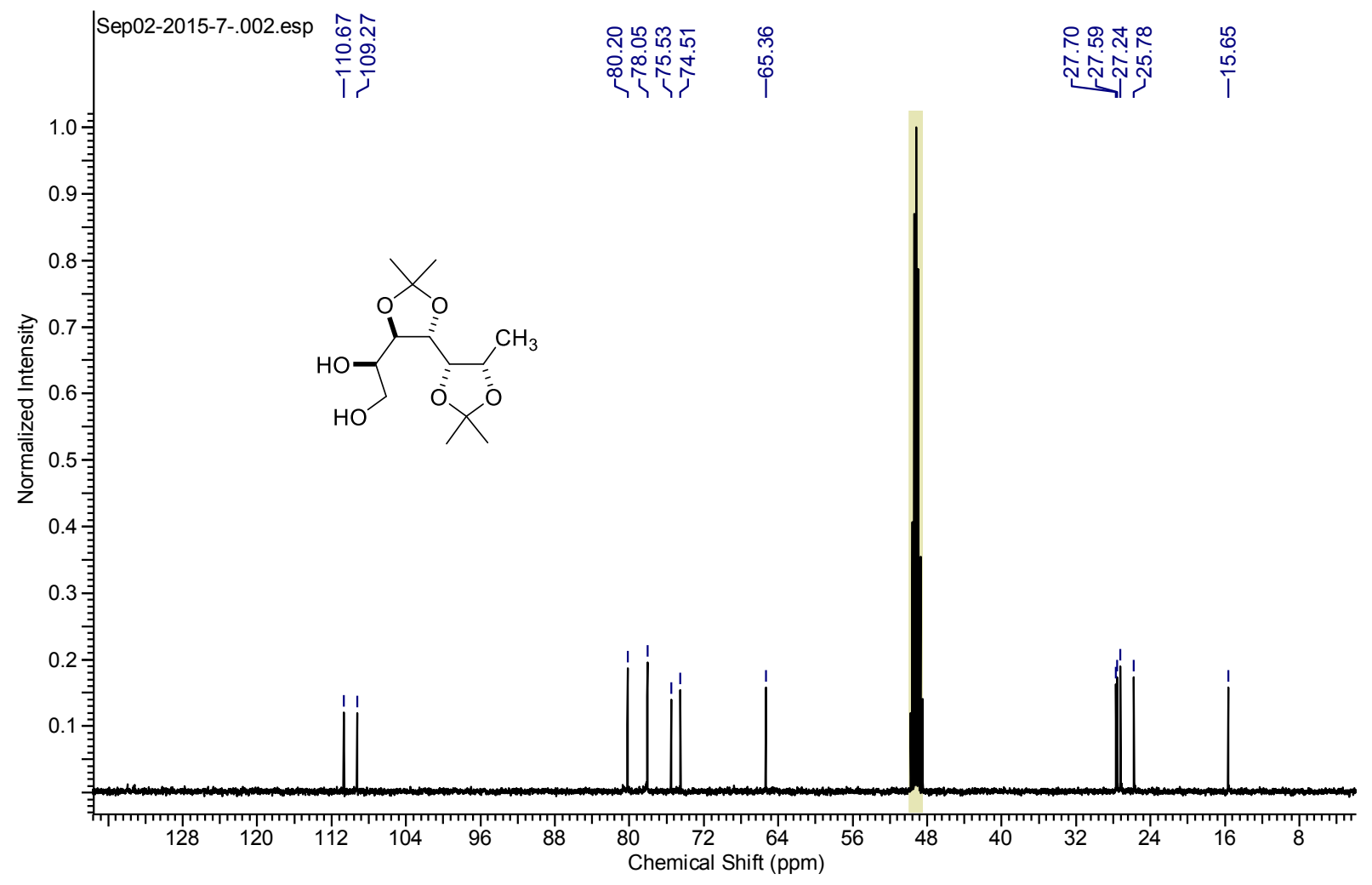


${ }^{1} \mathrm{H}$ NMR spectrum for $\mathbf{3 0}\left(\mathrm{CDCl}_{3}, 400 \mathrm{MHz}\right)$

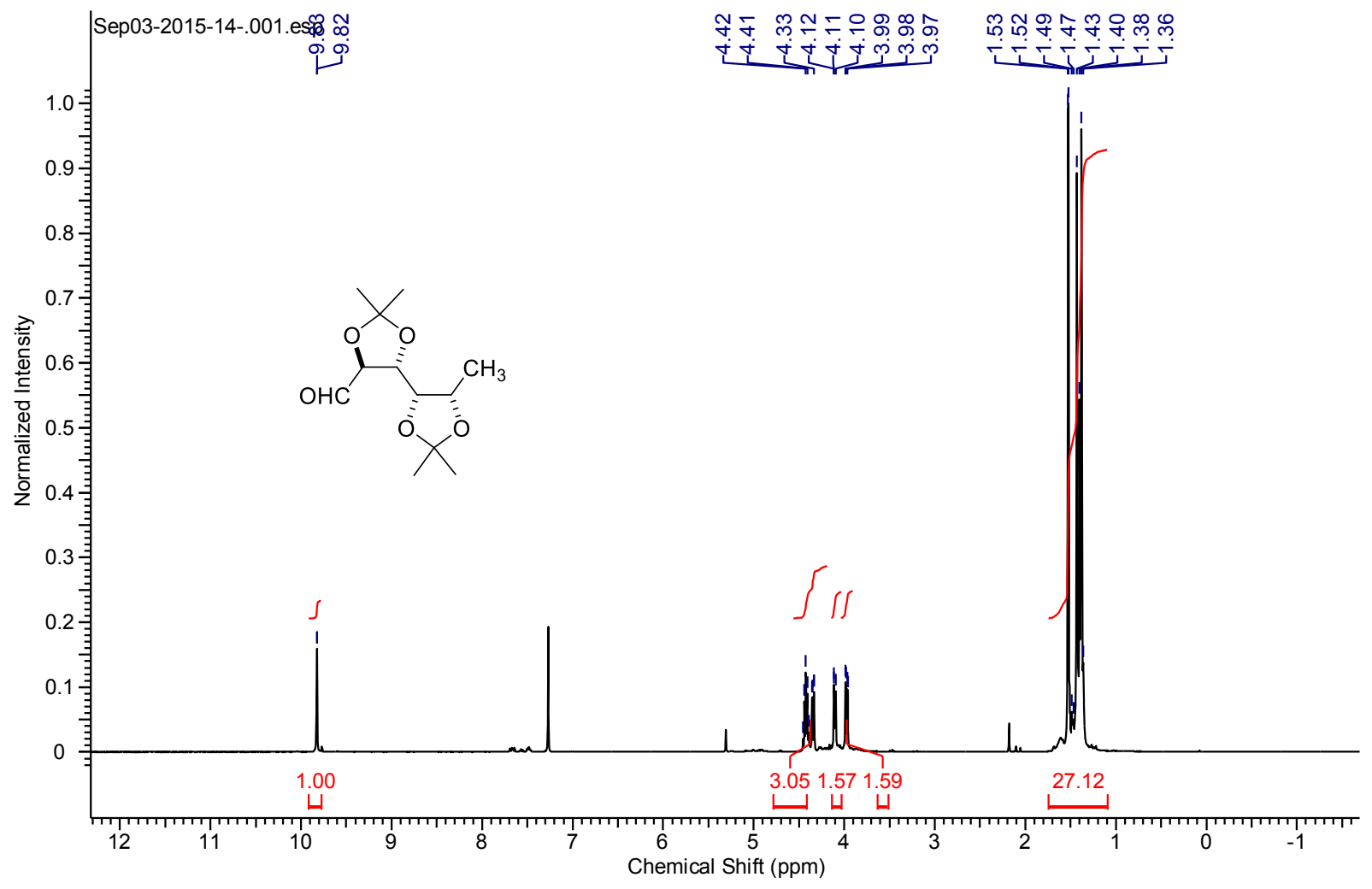


${ }^{13} \mathrm{C}$ NMR spectrum for $\mathbf{3 0}\left(\mathrm{CDCl}_{3}, 100 \mathrm{MHz}\right)$

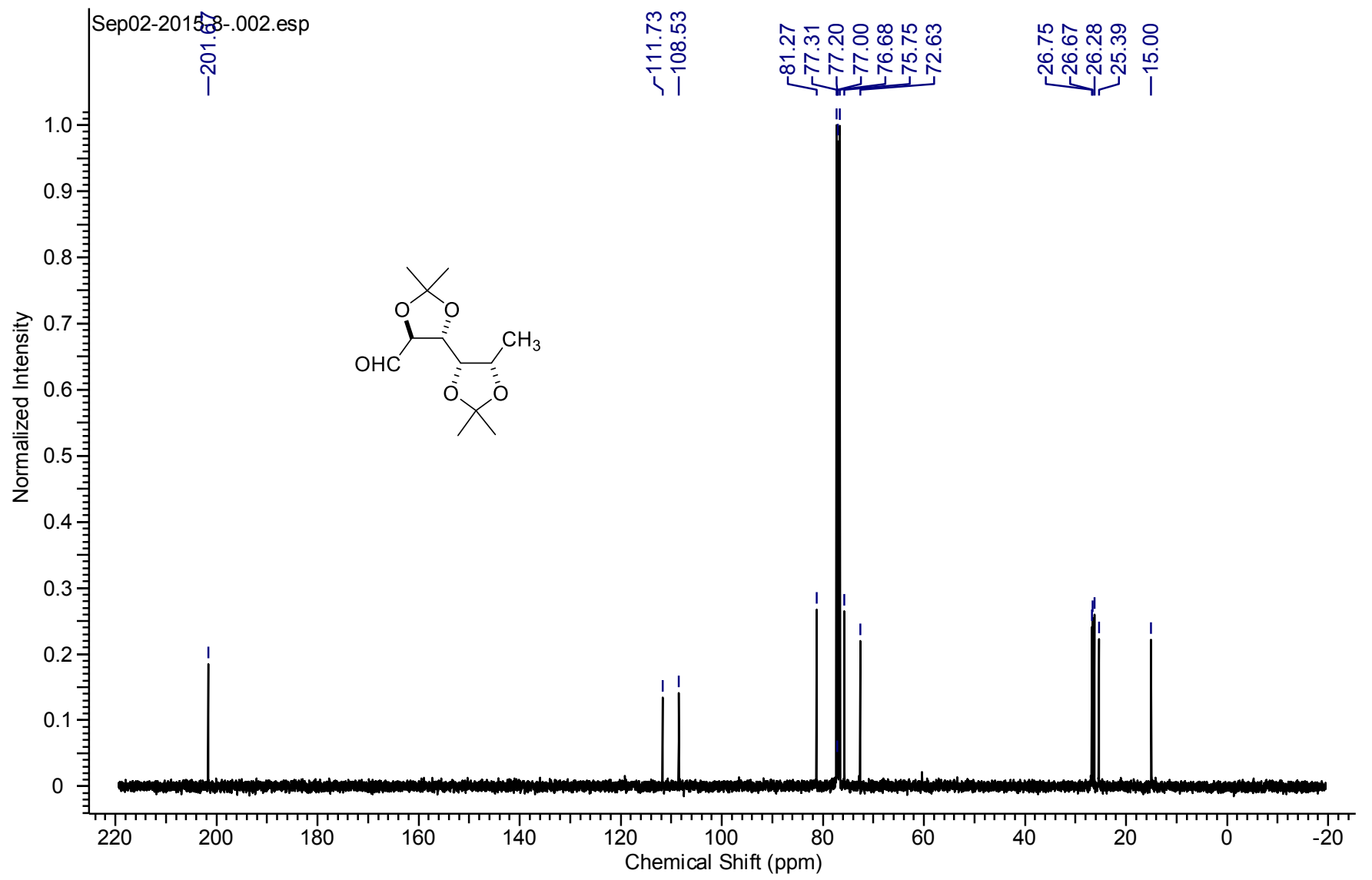


${ }^{1} \mathrm{H}$ NMR spectrum for 31 (before crystallization, $\mathrm{D}_{2} \mathrm{O}, 400 \mathrm{MHz}$ )

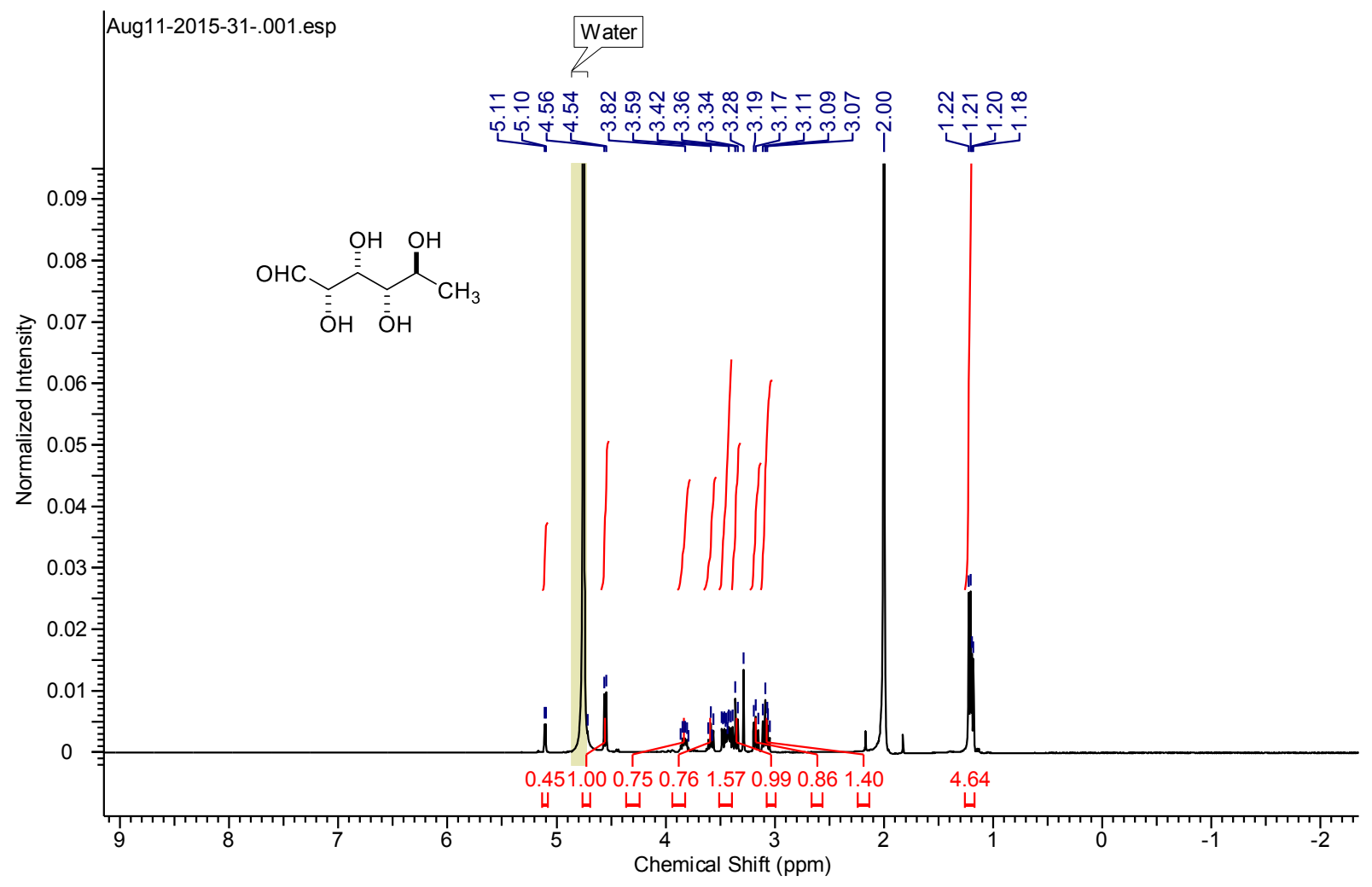


${ }^{1} \mathrm{H}$ NMR spectrum for 31 (after crystallization, $\mathrm{D}_{2} \mathrm{O}, 400 \mathrm{MHz}$ )

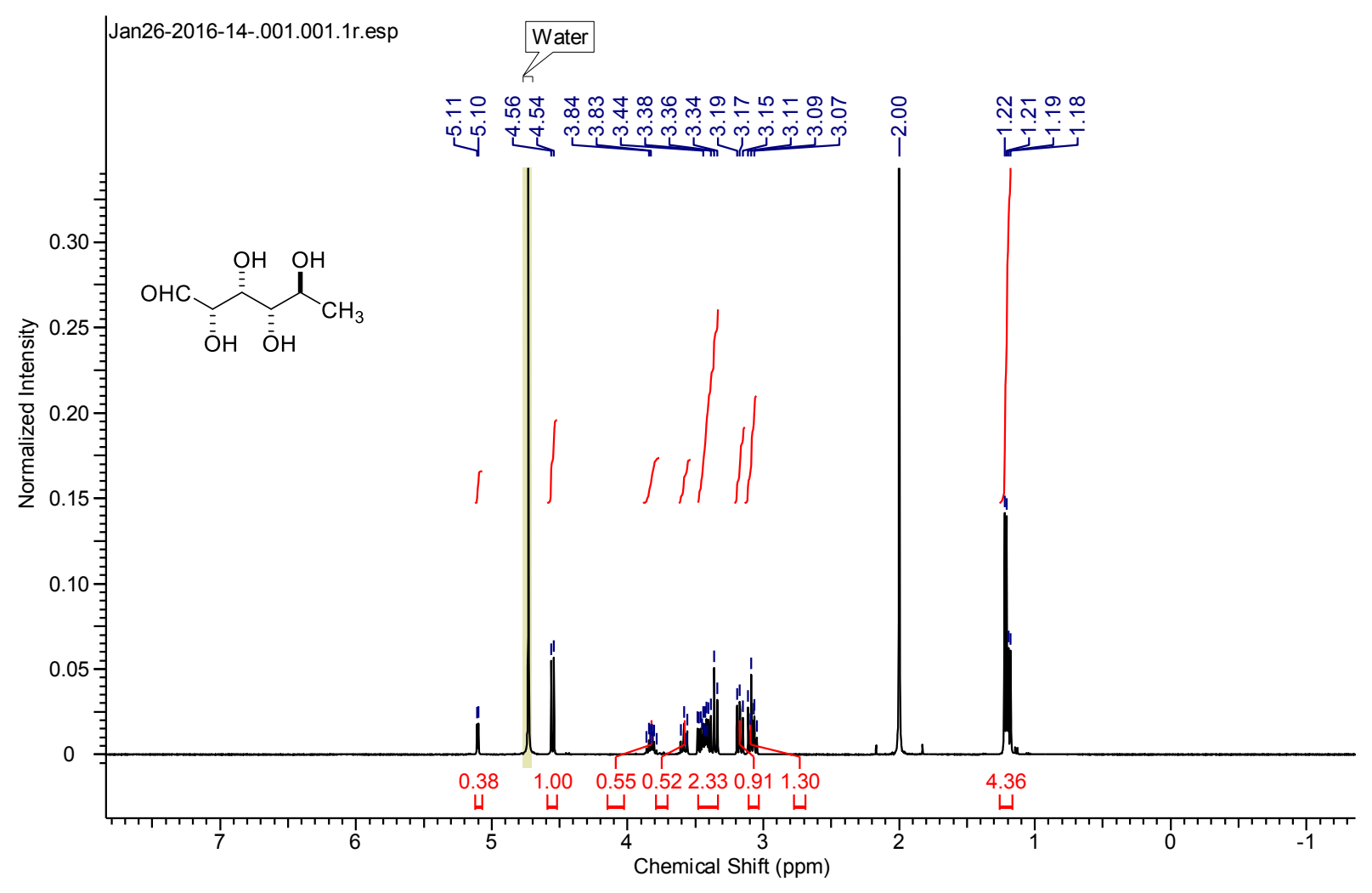


${ }^{13} \mathrm{C}$ NMR spectrum for 31 (after crystallization, $\mathrm{D}_{2} \mathrm{O}, 100 \mathrm{MHz}$ )

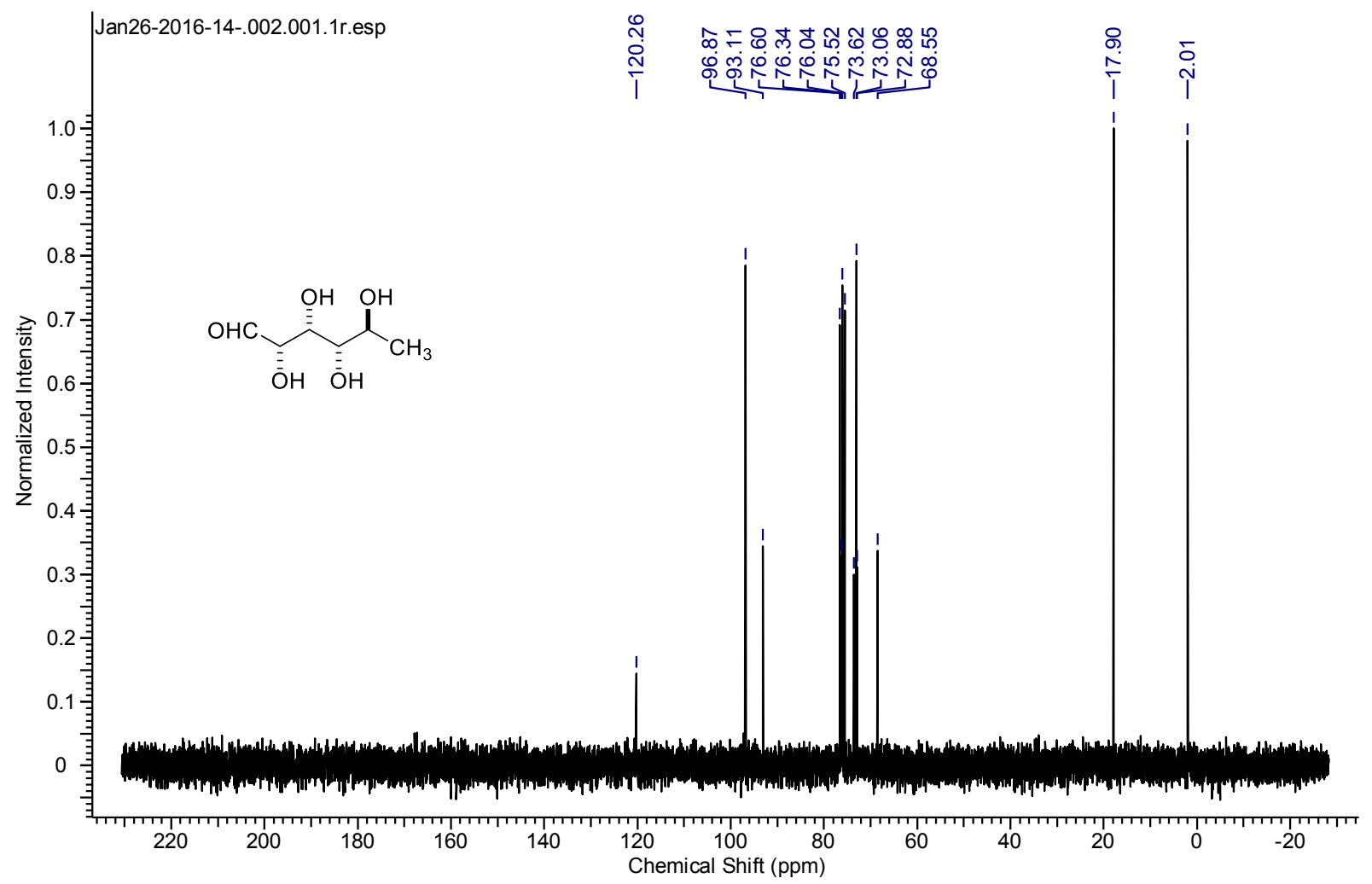


${ }^{1} \mathrm{H}$ NMR spectrum for commercial 6-deoxy-D-glucose $\left(\mathrm{D}_{2} \mathrm{O}, 400 \mathrm{MHz}\right)$

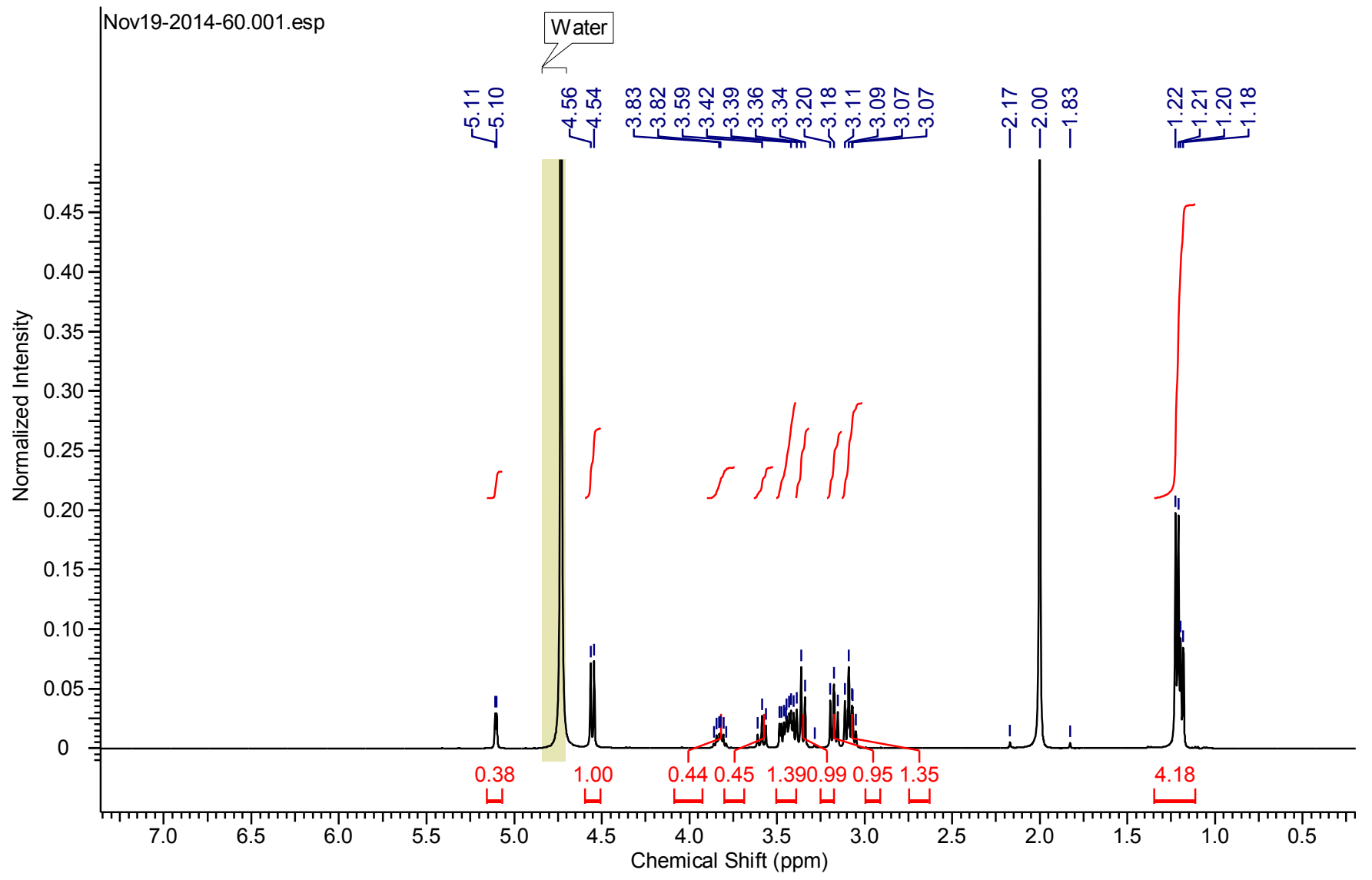


${ }^{1} \mathrm{H}$ NMR spectrum for $32\left(\mathrm{CDCl}_{3}, 400 \mathrm{MHz}\right)$

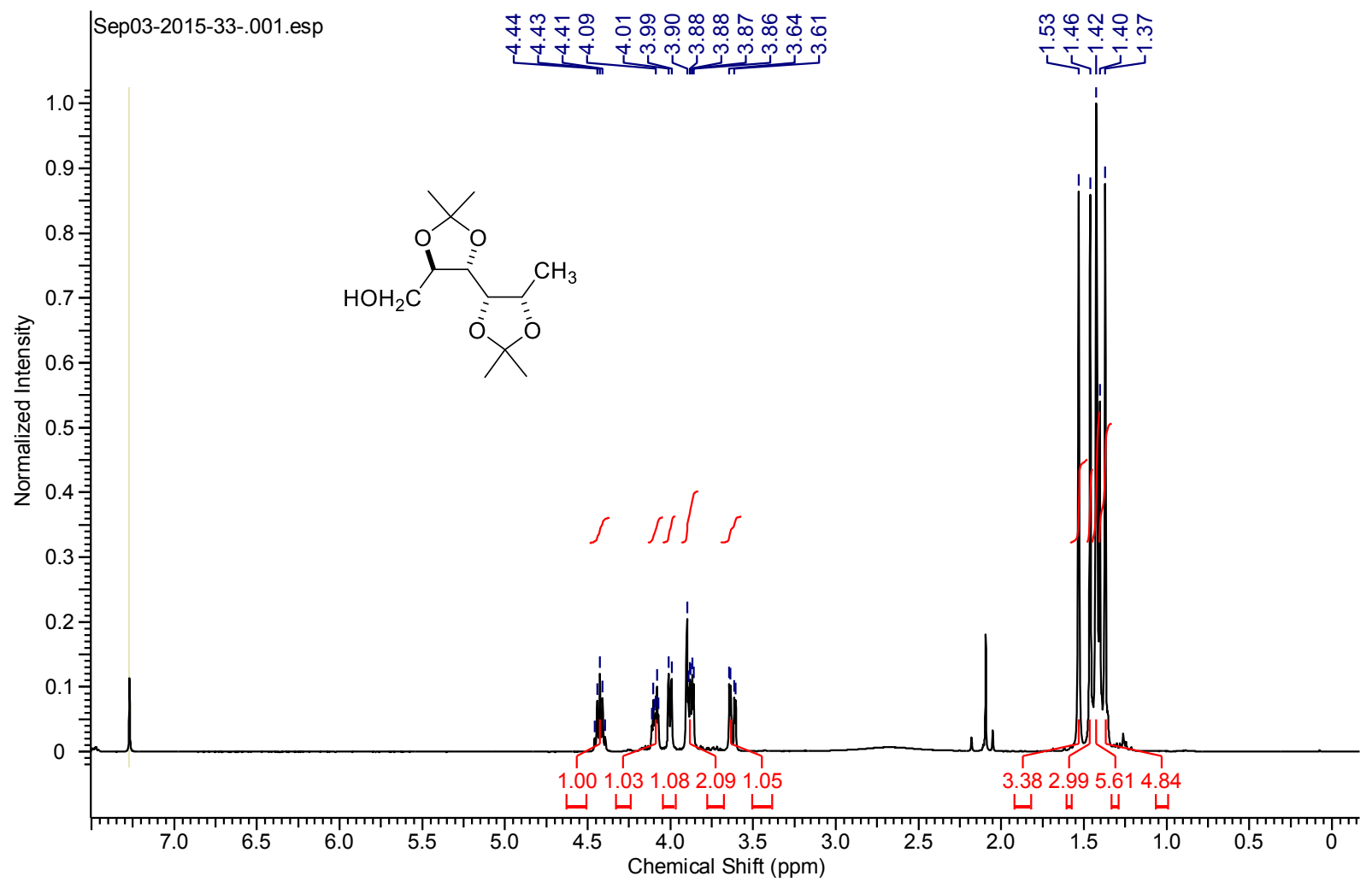


${ }^{13} \mathrm{C}$ NMR spectrum for $32\left(\mathrm{CDCl}_{3}, 100 \mathrm{MHz}\right)$

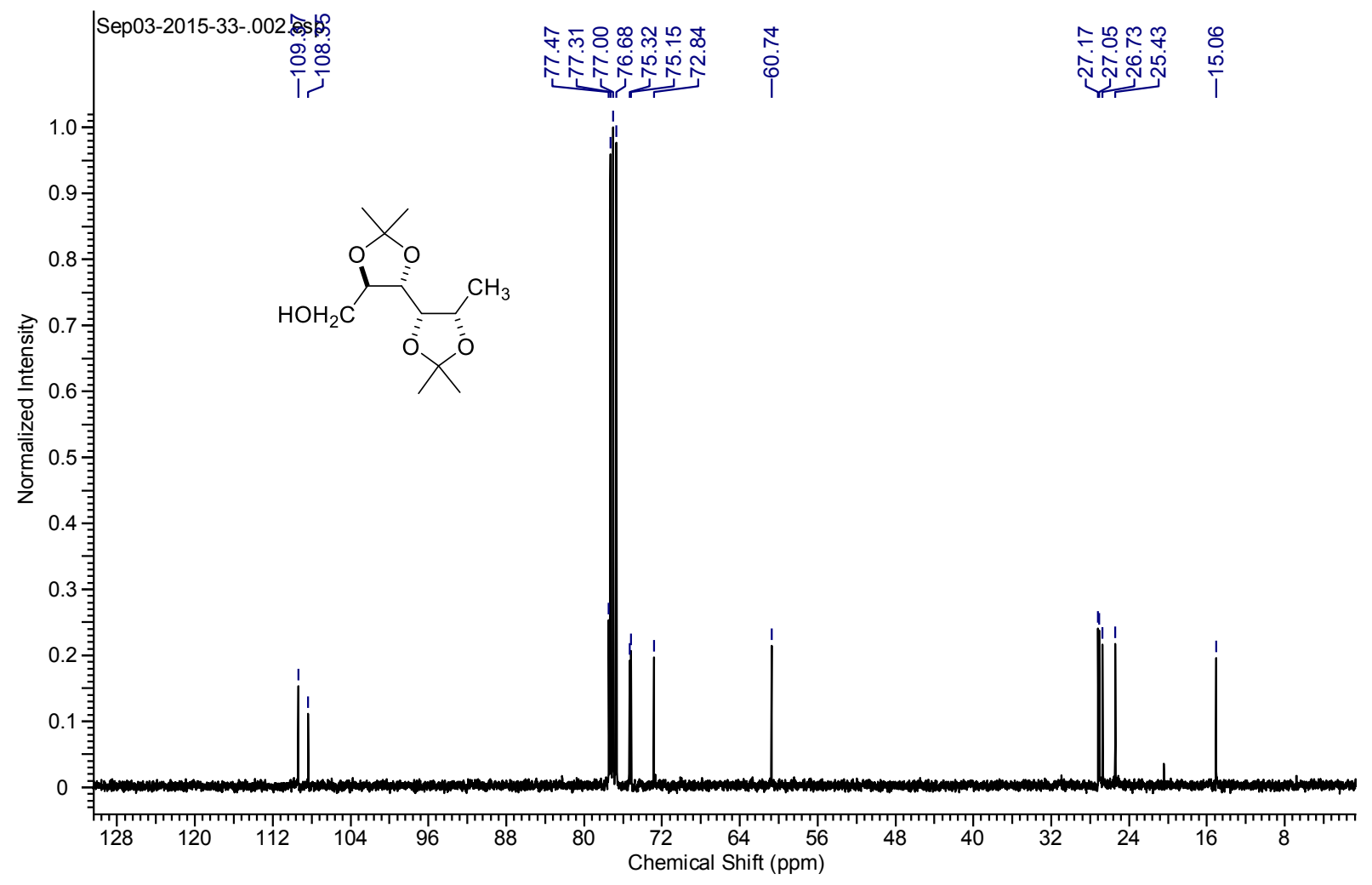


${ }^{1} \mathrm{H}$ NMR spectrum for $33\left(\mathrm{D}_{2} \mathrm{O}, 400 \mathrm{MHz}\right)$

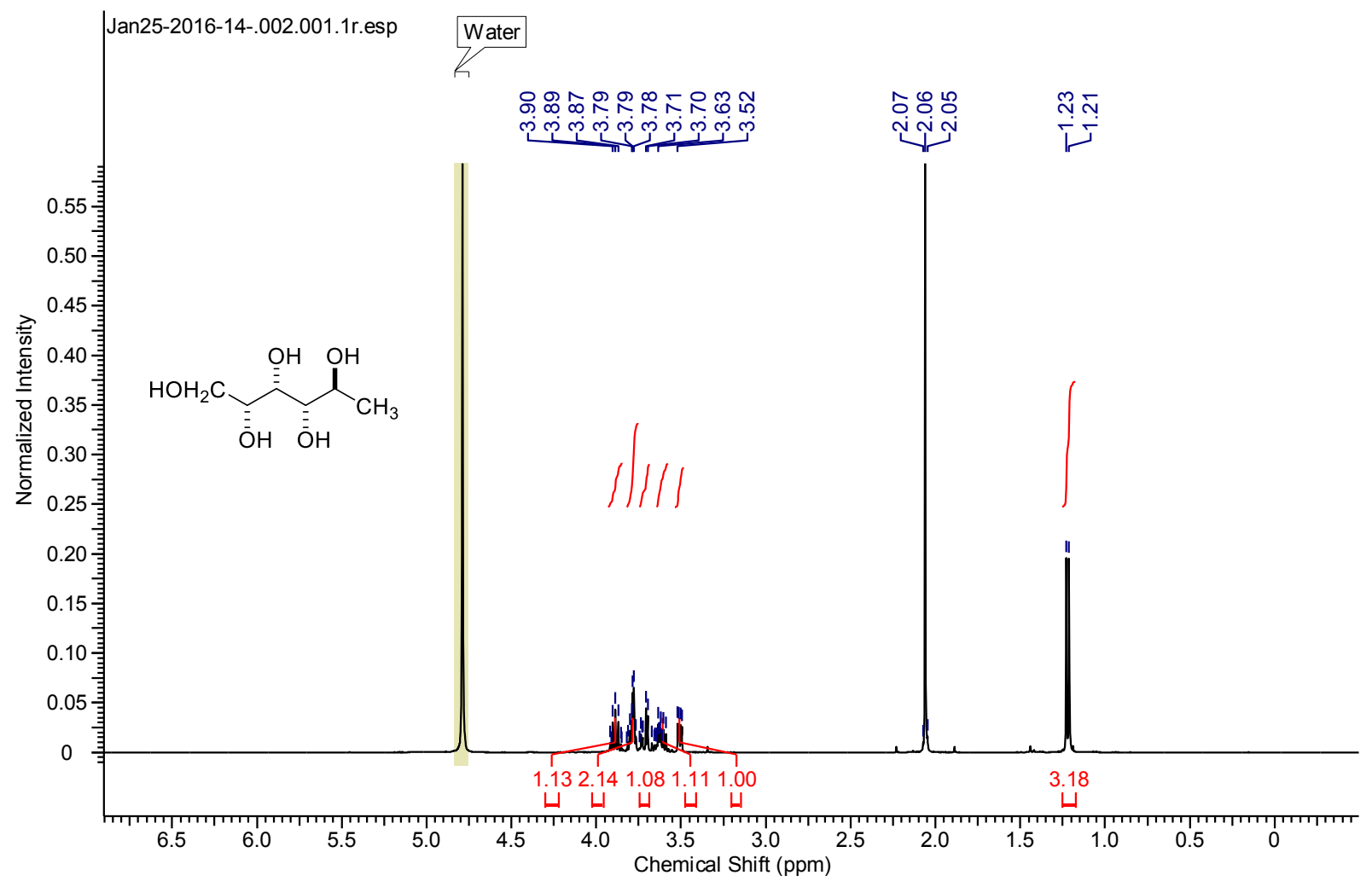


${ }^{13} \mathrm{C}$ NMR spectrum for $33\left(\mathrm{D}_{2} \mathrm{O}, 100 \mathrm{MHz}\right)$

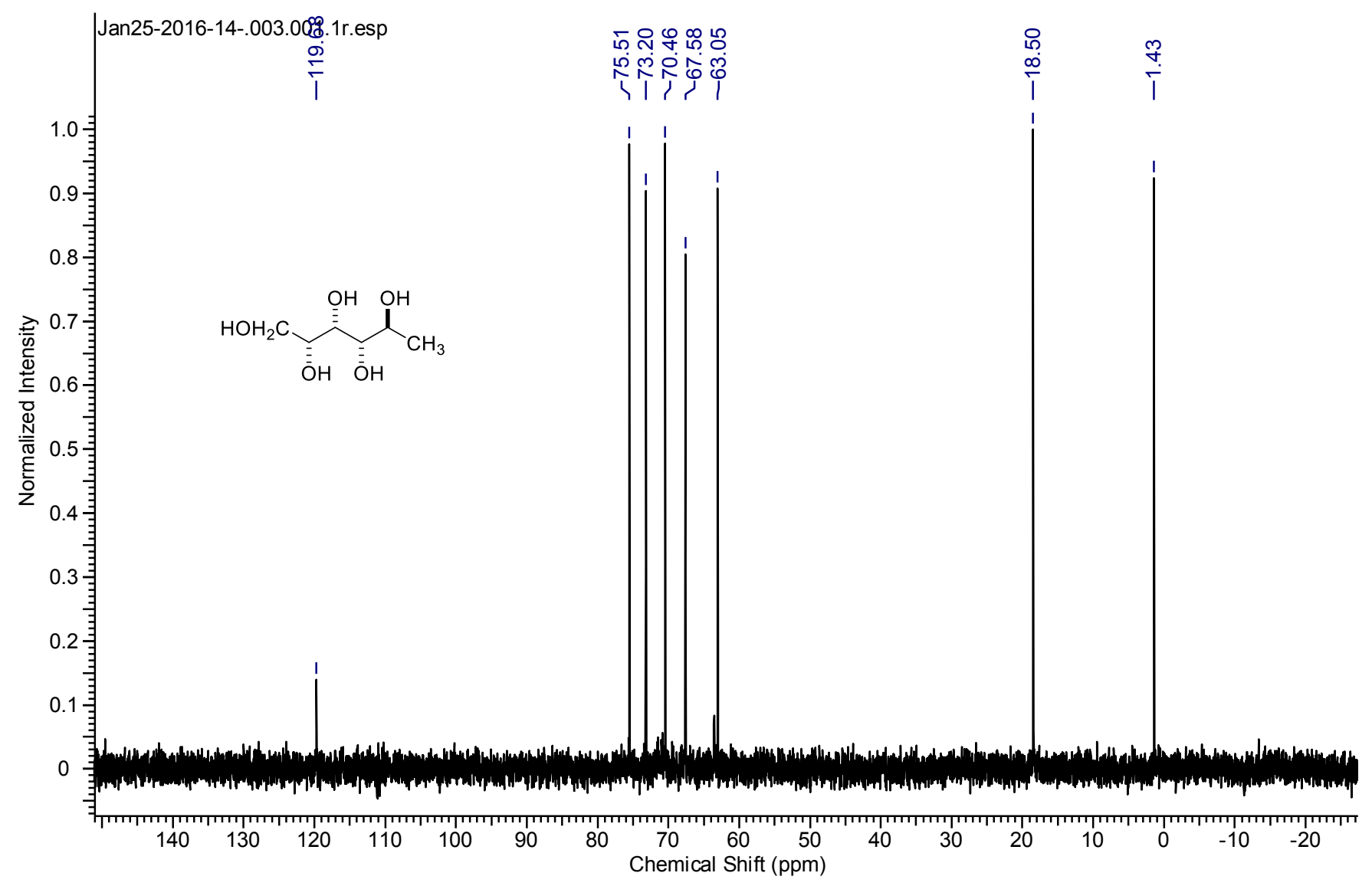

